\title{
Electroweak Symmetry Breaking and Beyond the Standard Model ${ }^{*}$
}

\author{
Tim Barklow \\ Stanford Linear Accelerator Center, Stanford, CA 94309 \\ Sally Dawson \\ Brookhaven National Laboratory, Upton, NY 11973
}

Howard E. Haber

Santa Cruz Institute for Particle Physics, Univ. of California, Santa Cruz, CA 95064

Jim Siegrist

Lawrence Berkeley Laboratory, University of California, Berkeley, CA 94720

* Chapter in "Report of the DPF Committee on Long Term Planning", Eds. Cahn, Cassel, Grannis, Peccei, Zeller, World Scientific

This manuscript has been authored under contract number DE-AC02-76CH00016 with the U.S. Department of Energy. Accordingly, the U.S. Government retains a non-exclusive, royalty-free license to publish or reproduce the published form of this contribution, or allow others to do so, for U.S. Government purposes.

\section{MASTER}




\section{DISCLAIMER}

Portions of this document may be illegible in electronic image products. Images are produced from the best available original document. 


\title{
Electroweak Symmetry Breaking and Beyond the Standard Model
}

\author{
TIM BARKLOW \\ Stanford Linear Accelerator Center, Stanford, CA 94309 \\ SALly DAWSON \\ Brookhaven National Laboratory, Upton, NY 11973
}

HOWARD E. HABER

Santa Cruz Institute for Particle Physics, Unversity of California, Santa Cruz, CA 95064

JIM SIEGRIST

Lawrence Berkeley Laboratory, University of California, Berkeley, CA 94720

\section{Working Group Members}

\begin{abstract}
H. Aihara,
H. Baer,

U. Baur,

R.S. Chivukula,

M. Cvetič,

A. Djouadi,

M. Drees,

S. Errede,

S. Godfrey,

M. Golden,

J.F. Gunion,

T. Han,

J.L. Hewett,

C.T. Hill,

D. London,

Lawrence Berkeley Laboratory

Florida State University

SUNY, Buffalo

Boston University

University of Pennsylvania

University of Montreal

University of Wisconsin

University of Illinois

Carleton University

Harvard University

University of California, Davis

University of California, Davis

Stanford Linear Accelerator Center

Fermilab

University of Montreal
\end{abstract}

T.W. Markiewicz,Stanford Linear Accelerator Center

\section{Introduction}

The development of the Standard Model of particle physics is a remarkable success story. Its many facets have been tested at present day accelerators; no significant unambiguous deviations have yet been found. In some cases, the model has been verified at an accuracy of better than one part in a thousand. This state of affairs presents our field with a challenge. Where do we go from here? What is our vision for future developments in particle physics? Are particle physicists' recent successes a signal of the field's impending demise, or do real long-term prospects exist for further progress? We assert that the long-term health and intellectual vitality of particle physics depends crucially on the development of a new generation of particle colliders that push the energy
S.P. Martin, University of Michigan

H. Murayama, Lawrence Berkeley Laboratory

J. Ng, TRIUMF, Vancouver BC

S.J. Parke, Fermilab

M.E. Peskin, Stanford Linear Accelerator Center

H. Pois, University of California, Davis

T.G. Rizzo, Stanford Linear Accelerator Center

R. Rosenfeld, Boston University

E.H. Simmons, Boston University

A. Stange, Brookhaven National Laboratory

T. Takeuchi, Fermilab

X. Tata, University of Hawaii

J. Terning, Boston University

S. Thomas, Stanford Linear Accelerator Center

S. Willenbrock, University of Illinois

D. Zeppenfeld, University of Wisconsin

frontier by an order of magnitude beyond present capabilities. In this report, we address the scientific issues underlying this assertion.

Despite the success of the Standard Model as a description of the properties of elementary particles, it is clear that the Standard Model is not a fundamental theory. Apart from any new physics phenomena that might lie beyond the Standard Model, one knows that the Standard Model neglects gravity. A more complete theory of elementary particle interactions (including gravity) contains the Planck scale $\left(M_{P} \simeq 10^{19} \mathrm{GeV}\right)$ as its basic energy scale. The Standard Model at best is a good lowenergy approximation to this more fundamental theory of particle interactions, valid in a limited domain of "lowenergies" of order $100 \mathrm{GeV}$ and below. In order to extend 
the Standard Model to higher energies, one must address three basic questions.

1. What is the complete description of the effective theory of fundamental particles at and below the electroweak scale?

In the Standard Model with one physical Higgs scalar, the scale of electroweak symmetry breaking is characterized by $v=246 \mathrm{GeV}$, the magnitude of the Higgs vacuum expectation value. More complicated models of particle physics can be easily constructed which closely approximate the Standard Model at present day energies, but have a much richer spectrum in the mass range above $100 \mathrm{GeV}$ and below a few $\mathrm{TeV}$ (henceforth referred to as the $\mathrm{TeV}$ scale). If one wishes to extrapolate physics from the electroweak scale and eventually deduce the nature of physics near the Planck scale, one must know the complete structure of the particle theory at the electroweak scale. The Standard Model possesses three generations of quarks and leptons (and no right-handed neutrinos) and an $\mathrm{SU}(3) \times \mathrm{SU}(2) \times \mathrm{U}(1)$ gauge group. Is that all? Might there be additional particles that populate the $\mathrm{TeV}$ scale? Some possibilities include: (i) an extended electroweak gauge group (e.g., extra U(1) factors, left-right (LR) symmetry as in $\mathrm{SU}(2)_{L} \times \mathrm{SU}(2)_{R} \times \mathrm{U}(1)$ models, etc.); (ii) new fermions (e.g., a fourth generation, mirror fermions, vector-like fermions, massive neutrinos, etc.); (iii) new bosons (CP-odd neutral and charged scalars in models with extended Higgs sectors, Goldstone or pseudo-Goldstone bosons, other exotic scalars, leptoquarks, vector resonances, etc.). Clearly, in order to extrapolate our particle theories to high energy with any confidence, one must know the complete $\mathrm{TeV}$-scale spectrum.

2. What is the mechanism for electroweak symmetry breaking?

The Standard Model posits that electroweak symmetry breaking (ESB) is the result of the dynamics of an elementary complex doublet of scalar fields. The neutral component of the scalar doublet acquires a vacuum expectation value and triggers ESB; the resulting particle spectrum contains three massive gauge bosons and the photon, massive quarks and charged leptons (and massless neutrinos) and a physical Higgs scalar. However, if one attempts to embed the Standard Model in a fundamental theory with a high energy scale $\left(M_{P}\right)$, then the masses of elementary scalars naturally assume values of order the high energy scale. This is due to the quadratic sensitivity of squared scalar masses to the high-energy scale of the underlying fundamental theory. In contrast, theoretical considerations (e.g. unitarity) require the mass of the Standard Model Higgs boson to be of order the electroweak scale. To accomplish this, one has three choices: (i) "Unnaturally" adjust the parameters of the highenergy theory (a fine tuning of 34 orders of magnitude in the scalar squared mass parameter is required) to produce the required light elementary scalar field.

(ii) Invoke supersymmetry to cancel quadratic sensitivity of the scalar squared masses to $M_{P}$. Electroweak symmetry breaking is triggered by the dynamics of a weakly-coupled Higgs sector.

(iii) Elementary Higgs scalars do not exist. A Higgs-like scalar state (if it exists) would reveal its composite nature at the $\mathrm{TeV}$-scale, where new physics beyond the Standard Model enters. Electroweak symmetry breaking is triggered by non-perturbative strong forces.

In order to implement (ii), one must first note that supersymmetry is not an exact symmetry of nature (otherwise, all known particles would have equal-mass supersymmetric partners). If supersymmetry is to explain why the Higgs boson mass is of order the electroweak scale, then supersymmetry-breaking effects which split the masses of particles and their super-partners must be roughly of the same order as the electroweak scale. Thus, if supersymmetry is connected with the origin of ESB, one expects to discover a new spectrum of particles and interactions at the $\mathrm{TeV}$-scale or below. In addition, such models of "low-energy" supersymmetry are compatible with the existence of weakly-coupled elementary scalars with masses of order the ESB-scale. No direct experimental evidence for the supersymmetric particle spectrum presently exists. But there is tantalizing indirect evidence. Starting from the known values of the SU(3), $\mathrm{SU}(2)$, and $\mathrm{U}(1)$ gauge couplings at $m_{Z}$ and extrapolating to high energies, one finds that the three gauge couplings meet at a single point if one includes the effects of supersymmetric particles (with masses at or below $1 \mathrm{TeV}$ ) in the running of the couplings. Unification of couplings then takes place at around $10^{16} \mathrm{GeV}$, only a few orders of magnitude below $M_{P}$. The three gauge couplings do not meet at a single point if only Standard Model particles contribute to the running of the couplings. This could be a hint for low-energy supersymmetry, and suggests that the theory of fundamental particles remains weakly coupled and perturbative all the way up to energies near $M_{P}$.

New physics is also invoked to explain the origin of electroweak symmetry breaking in choice (iii). For example, in technicolor models (which make use of the mechanism analogous to the one that is responsible for chiral symmetry breaking in QCD), electroweak symmetry breaking occurs when pairs of techni-fermions condense in the vacuum. One then identifies a new scale, $\Lambda_{E S B} \simeq 4 \pi v \sim \mathcal{O}(1 \mathrm{TeV})$, where new physics beyond 
the Standard Model must enter. Other approaches, such as effective Lagrangian descriptions of the strongly interacting Higgs sector, preon models, top-mode condensate models, composite Higgs models, etc., also fall into this category. Unfortunately, due to the presence of nonperturbative strong forces, it is often difficult to make reliable detailed computations in such models. Moreover, phenomenological difficulties inherent in the simplest examples often require additional structure (e.g., an extended technicolor sector is needed in technicolor models to generate fermion masses). Unfortunately, a completely phenomenologically viable fundamental model of strongly-coupled ESB has not yet been constructed.

There is one important theoretical lesson that one should draw from the above remarks. Namely, the nature of ESB dynamics must be revealed at or below the 1 TeV energy scale. This is not a matter of conjecture. The relevant scale is implicitly built into the Standard Model through $v \equiv 2 m_{W} / g$. If one wishes to take the most conservative approach, one would say that the study of the $\mathrm{TeV}$ scale must reveal the Higgs boson, but with no guarantees of anything beyond. However, if one takes the naturalness argument seriously, it follows that the study of the $\mathrm{TeV}$ scale must reveal a new sector of physics beyond the Standard Model responsible for ESB dynamics-either a super-particle spectrum or a new strongly-interacting sector of fermions, scalars, and/or vector resonances.

3. What is the origin of the Standard Model parameters?

I.I. Rabi asked the famous question concerning the unexpected discovery of the muon: "Who ordered that?!" In the context of the Standard Model, we ponder the generalizations of Rabi's question: (i) Why are there three generations? [or what is the origin of flavor?]; (ii) Where do the quark and lepton masses come from? [or what is the origin of the fermion mass matrices?]; (iii) What is the origin of CP-violation? [or is the phase in the Cabibbo-Kobayashi-Maskawa (CKM) mass matrix the only source of CP-violation? and why is $\theta_{Q C D}$, which would give rise to CP-violation from the strong interactions, so small?]. Note that although the minimal supersymmetric extension of the Standard Model addresses the origin of electroweak symmetry breaking, it provides little insight into any of the above questions. In fact, one would simply add yet another question: (iv) What is the origin of the (soft)-supersymmetry-breaking parameters that characterize the model of low-energy supersymmetry?

In contrast to the problem of ESB discussed above, the Standard Model does not provide any clues as to the energy scale responsible for addressing the origin of the Standard Model parameters (or a supersymmetric extension thereof). The relevant energy scale could lie any- where from $1 \mathrm{TeV}$ to $M_{P}$. For example, in conventional theories of low-energy supersymmetry, the origin of the Standard Model and supersymmetric parameters lies at or near the Planck scale. In contrast, theories of extended technicolor typically attempt to solve the flavor problem at energy scales below $100 \mathrm{TeV}$. The latter approach is more ambitious; perhaps this is one reason why no compelling model of this type has been constructed. Nevertheless, any model of flavor and fermion masses requires a definite framework for electroweak symmetry breaking. Thus, the elucidation of $\mathrm{TeV}$-scale physics will have significant ramifications for our understanding of physics at even higher energies.

Other future particle physics experiments may also address some of these issues. $B$-factories will thoroughly test the CKM-parametrization of CP-violation. Any evidence for new sources of CP-violation would constitute physics beyond the Standard Model. The next generation of neutrino mixing and mass experiments will try to verify recent experimental hints for massive neutrinos. If massive neutrinos are confirmed, then the Standard Model will require an extension. The simplest possibility is to include right-handed neutrino states. Majorana mass terms for the right-handed neutrinos are $\mathrm{SU}(2) \times \mathrm{U}(1)$ conserving and therefore constitute a completely new energy scale $\left(M_{R}\right)$ characterizing the extended Standard Model. At present, $M_{R}$ is undetermined and can lie anywhere between the electroweak scale and $M_{P}$. If $M_{R} \gg m_{Z}$, then the effect of this new scale will be simply to add the neutrino mass and mixing parameters to the list of Standard Model parameters whose origin will be difficult to discern directly from particle physics experiments in the foreseeable future.

In this sense, the search for electroweak symmetry breaking presents the unique guarantee - the dynamics that generates ESB must be revealed by the next generation of colliders that can directly explore the TeV-scale. Thus, it is crucial to explore thoroughly the TeV energy scale in order to elucidate the dynamics of electroweak symmetry breaking and the associated fundamental particle spectrum. This program must be a primary focus of particle physics research in the long-term future plans of the field.

In this report, we explore the capabilities of the approved future facilities and a variety of hypothetical future machines, summarized in Table 1 . We have also begun to study the physics opportunities at more speculative future colliders, such as a $60 \mathrm{TeV}$ hadron supercollider (which would supersede the LHC) and a multi-TeV $\mu^{+} \mu^{-}$collider, although the overall capabilities of these machines have yet to be addressed in a comprehensive fashion.

In this report, we do not directly address the origin of the Standard Model parameters. Nor do we address the possibility that new and interesting physics beyond the 
Table 1:

\begin{tabular}{|lrcc|}
\hline \multicolumn{1}{|c}{ Name } & Type & $\sqrt{s}$ & Yearly $\int \mathcal{L} d t$ \\
\hline Approved Projects & & & \\
LEP-II & $e^{+} e^{-}$ & $\sim 190 \mathrm{GeV}$ & $\sim 100 \mathrm{pb}^{-1}$ \\
Tevatron (Main & & & \\
$\quad$ Injector) & $p \bar{p}$ & $2 \mathrm{TeV}$ & $1 \mathrm{fb}^{-1}$ \\
LHC & $p p$ & $14 \mathrm{TeV}$ & $10-100 \mathrm{fb}^{-1}$ \\
\hline Tevatron Upgrades (under consideration) \\
TeV & $p \bar{p}$ & $2 \mathrm{TeV}$ & $10 \mathrm{fb}^{-1}$ \\
Di-Tevatron & $p \bar{p}$ & $4 \mathrm{TeV}$ & $20 \mathrm{fb}^{-1}$ \\
\hline$e^{+} e^{-}$Linear Collider & & \\
JLC, NLC, TESLA & $e^{+} e^{-\dagger} 0.5-1.5 \mathrm{TeV}$ & $50-200 \mathrm{fb}^{-1}$ \\
& $\left(^{\dagger}\right.$ with $e \gamma, \gamma \gamma, e^{-} e^{-}$options) \\
\hline
\end{tabular}

Standard Model might populate the energy interval above the $\mathrm{TeV}$-scale. We focus directly on $\mathrm{TeV}$-scale physics accessible to the next generation of colliders. Namely, we examine the requirements for completing the $\mathrm{TeV}$ scale particle spectrum, and for unraveling the dynamics of electroweak symmetry breaking. Particularly in the latter case, we expect that future colliders will discover new physics beyond the Standard Model (BSM) associated with this dynamics. Ten ESB\&BSM subgroups were established to address these issues. Two additional subgroups were added to examine experimental issues associated with the search for ESB\&BSM physics at future hadron and $e^{+} e^{-}$colliders, respectively. Conveners of the twelve subgroups prepared executive summaries which form the basis of this document. More detailed expositions will be published separately in Ref. [1], which will also contain a more comprehensive list of references.

The subgroups and their conveners are listed below. Three subgroups addressed the case of weakly-coupled ESB dynamics:

- Weakly-Coupled Higgs Bosons (J.F. Gunion, A. Stange, and S. Willenbrock)

- Low-Energy Supersymmetry-Implications of Models (M. Drees, S.P. Martin, and H. Pois)

- Low-Energy Supersymmetry Phenomenology (H. Baer, H. Murayama, and X. Tata)

Two subgroups addressed the case of strongly-coupled ESB dynamics:

- Strongly-Coupled ESB Sector-Model Independent Study (M. Golden and T. Han)

- Strongly-Coupled ESB Sector-Implications of Models (R.S. Chivukula, R. Rosenfeld, E.H. Simmons, and J. Terning)
Two subgroups addressed the question of identifying the interactions and full spectrum of the $\mathrm{TeV}$-scale particle theory:

- New Gauge Bosons Beyond the $W^{ \pm}$and $Z$ (M. Cvetič and S. Godfrey)

- New Particles and Interactions (A. Djouadi, J. Ng and T.G. Rizzo)

Three subgroups addressed the search for ESB\&BSM physics via precision measurements of Standard Model processes:

- Anomalous Gauge Boson Couplings (H. Aihara, U. Baur, D. London and D. Zeppenfeld)

- Top-Quarks as a Window to Electroweak Symmetry Breaking (S.J. Parke, C.T. Hill and M.E. Peskin)

- Virtual Effects of New Physics (J.L. Hewett, T. Takeuchi, and S. Thomas)

Two subgroups focussed on the interplay of ESB\&BSM physics and detector and machine issues for future colliders:

- Experimental Issues in the Search for ESB\&BSM at Future Hadron Colliders (S. Errede and J. Siegrist)

- Experimental Issues in the Search for ESB\&BSM at Future $e^{+} e^{-}$Colliders (T. Barklow and T.W. Markiewicz)

\section{Weakly-Coupled Higgs Bosons}

\subsection{The Standard Model and MSSM Higgs Sectors [2]}

In the Standard Model (SM), the electroweak symmetry is broken by an elementary weak doublet of scalar fields, whose neutral component acquires a non-zero vacuum expectation value. This model predicts the existence of a single CP-even neutral scalar called the Higgs boson, $H_{S M}$, of unknown mass but with fixed couplings to other particles proportional to their masses. This is the minimal model of electroweak symmetry breaking. Detailed studies of the phenomenological profile of $H_{S M}$ are important, since they provide significant benchmarks for any experimental search for electroweak symmetry breaking.

The underlying physics responsible for electroweak symmetry breaking is only weakly constrained by present-day experimental data. Thus, in the search for electroweak symmetry breaking, one must be open to more complicated possibilities. For example, it is easy to generalize the minimal Higgs model by simply adding additional scalar doublets. Models of this type posiess additional CP-even neutral scalars, new CP-odd neurral 
scalars, and charged scalars. One of the simplest extensions of the SM Higgs sector is the two-Higgs-doublet model (with a CP-invariant scalar potential). The scalar spectrum of this model contains two CP-even neutral scalars, $h^{0}$ and $H^{0}$ (with $m_{h^{\circ}} \leq m_{H^{0}}$ ), one CP-odd neutral scalar, $A^{0}$, and a charged Higgs pair, $H^{ \pm}$. The importance of this model will be clarified below.

In Section 1, a theoretical overview was presented of the possible mechanisms that could be responsible for electroweak symmetry breaking. The Standard Model with its minimal Higgs sector or with an extended Higgs sector (as described above) is theoretically unsatisfactory, since there is no "natural" way of understanding the origin of the electroweak symmetry breaking scale. Two generic solutions to this theoretical problem have been put forward. In the first solution, the Standard Model is extended to include supersymmetric partners. In the second solution, new strongly-interacting forces are invoked, whose dynamics are responsible for triggering electroweak symmetry breaking. In the former case, the Higgs bosons are elementary weakly-coupled scalars, while in the latter case, any physical scalar state is probably strongly-coupled and composite. The supersymmetric approach is treated in Sections 3 and 4, while the strongly-coupled scalar sector is considered in Sections 5 and 6 .

The focus of this section is the search for weaklycoupled elementary Higgs bosons. In the Standard Model, the Higgs self-coupling is proportional to the square of the Higgs mass. For Higgs masses below (roughly) $700 \mathrm{GeV}, H_{S M}$ is a weakly-coupled scalar. In supersymmetric models, the Higgs bosons are also elementary and weakly-coupled. Moreover, as reviewed in Section 3, the Higgs sector of the minimal supersymmetric extension of the Standard Model (MSSM) consists of two scalar doublets (interacting via a CP-conserving potential). Thus, the MSSM Higgs spectrum consists of $h^{0}, H^{0}, A^{0}$, and $H^{ \pm}$, introduced above. As a result, the phenomenology of this Higgs sector is particularly important, since it introduces new kinds of Higgs phenomena that can be observed in future experiments, while being a theoretically well-motivated model for the scalar sector associated with electroweak symmetry breaking.

This section considers the prospects for the discovery of weakly-coupled Higgs bosons at present and future colliders. Both the SM Higgs boson, $H_{S M}$, and the scalars of the MSSM Higgs sector are considered. Some aspects of the phenomenology of $h^{0}, H^{0}, A^{0}$, and $H^{ \pm}$ do not strongly depend on the fact that they arise in a supersymmetric model. However, the general parameter space of an arbitrary two-Higgs doublet model is quite large (with four independent mass parameters and at least two other additional dimensionless parameters). In contrast, the two-doublet Higgs sector of the MSSM possesses numerous relations among Higgs masses and couplings due to the underlying supersymmetry. In particular, the MSSM tree-level Higgs masses and couplings are determined in terms of two free parameters: $m_{A^{\circ}}$ o and $\tan \beta \equiv v_{2} / v_{1}$ [where $v_{2}\left(v_{1}\right)$ is the vacuum expectation value of the Higgs field that couples to up-type (down-type) fermions]. One remarkable consequence of supersymmetry is that the mass of the lightest CP-even neutral scalar, $h^{0}$, is bounded from above by a mass value of order $m_{Z}$. However, in obtaining the precise upper bound, there are important radiative corrections that cannot be neglected. Through the radiative corrections, the Higgs masses also acquire dependence on the details of the MSSM spectrum. The most important piece of these corrections depends primarily on the masses of the top-quark (taken here to be $m_{t}=175 \mathrm{GeV}$ ) and its supersymmetric partners [3]. To be conservative, in this section the effects of the radiative corrections have been maximized by assuming that all supersymmetric particle masses (and mass mixing parameters) are of order $1 \mathrm{TeV}$. In this case, the light CP-even Higgs boson mass is bounded by $m_{h^{\circ}} \leq m_{h^{\circ}}^{\max } \simeq 120 \mathrm{GeV}$. The following properties of the MSSM Higgs sector are noteworthy. If $m_{A^{0}} \gg m_{Z}$, then $m_{A^{0}} \simeq m_{H^{\circ}} \simeq m_{H^{ \pm}}$(in addition, if $\tan \beta \gg 1$ then $m_{h^{0}} \simeq m_{h^{0}}^{\max }$ ). Moreover, the couplings of $h^{0}$ to fermions and gauge bosons are indistinguishable from those of the Standard Model Higgs boson, while the couplings $H^{0} W^{+} W^{-}, H^{0} Z Z$ and $Z A^{0} h^{0}$ are severely suppressed. Finally, assuming $\tan \beta>1$, the couplings of $A^{0}$ and $H^{0}$ to $b \bar{b}$ and $\tau^{+} \tau^{-}(t \bar{t})$ are enhanced (suppressed). In contrast, for the case $m_{A^{0}} \lesssim \mathcal{O}\left(m_{Z}\right)$ and $\tan \beta>1, A^{0}$ and $h^{0}$ exhibit the latter coupling pattern to fermions. The couplings $h^{0} W^{+} W^{-}, h^{0} Z Z$ and $Z A^{0} H^{0}$ are severely suppressed, while the $H^{0}$ couplings to fermions and gauge bosons are SM-like.

We now outline the capabilities of present and proposed future colliders to search for the SM Higgs boson and the MSSM Higgs bosons [2]. The SM Higgs discovery limits for the most useful discovery modes are summarized in Table 2. The corresponding MSSM Higgs discovery limits are summarized in Table 3 . For $b$-tagged modes, a tagging efficiency of $30 \%$ and purity of $1 \%$ are assumed. Since the MSSM Higgs discovery limits are rather complex, additional information is provided here to assist the reader in interpreting the results presented in Table 3. The MSSM Higgs sector is most conveniently parametrized in terms of $m_{A^{0}}$ and $\tan \beta$. All values of $m_{A^{0}} \leq 1000 \mathrm{GeV}$ and $1 \leq \tan \beta \leq 60$ have been surveyed (as preferred in the MSSM). There is also some dependence of the discovery limits on the radiative corrections, the main effect of which is to increase the mass of $h^{0}$ above its tree-level value. The discovery regions of Table 3 have been obtained assuming that the effects of the radiative corrections are maximal, as noted above.

A few examples are now given to clarify the meanng of the discovery regions presented in Table 3 . At L.F.' II 
Table 2: Standard Model Higgs boson discovery modes. All masses are specified in GeV.

\begin{tabular}{|l|c|c|}
\hline Machine $\left(\sqrt{s}, \int \mathcal{L} d t\right)$ & Mode & Discovery Region \\
\hline LEP-I & $e^{+} e^{-} \rightarrow Z^{*} H_{S M}$ & $m_{H_{S M}} \lesssim 65$ \\
\hline LEP-II, $\left(176 \mathrm{GeV}, 500 \mathrm{pb}^{-1}\right)$ & $e^{+} e^{-} \rightarrow Z H_{S M}$ & $m_{H_{S M}} \lesssim 80$ \\
\hline LEP-II, $\left(190 \mathrm{GeV}, 500 \mathrm{pb}^{-1}\right)$ & $e^{+} e^{-} \rightarrow Z H_{S M}$ & $m_{H_{S M}} \lesssim 95$ \\
\hline Tevatron, $\left(2 \mathrm{TeV}, 5 \mathrm{fb}^{-1}\right)$ & $p \bar{p} \rightarrow W H_{S M} ; H_{S M} \rightarrow b \bar{b}$ & $m_{H_{S M}} \lesssim 60-80$ \\
\hline TeV*,$\left(2 \mathrm{TeV}, 30 \mathrm{fb}^{-1}\right)$ & $p \bar{p} \rightarrow W H_{S M} ; H_{S M} \rightarrow b \bar{b}$ & $m_{H_{S M}} \lesssim 95$ \\
& $p \bar{p} \rightarrow W H_{S M} ; H_{S M} \rightarrow \tau^{+} \tau^{-}$ & $110 \lesssim m_{H_{S M}} \lesssim 120$ \\
\hline DiTeV,$\left(4 \mathrm{TeV}, 30 \mathrm{fb}^{-1}\right)$ & $p \bar{p} \rightarrow W H_{S M} ; H_{S M} \rightarrow b \bar{b}$ & $m_{H_{S M}} \lesssim 95$ \\
& $p \bar{p} \rightarrow Z Z \rightarrow 4 \ell$ & $m_{H_{S M}} \sim 200$ \\
\hline LHC, $\left(14 \mathrm{TeV}, 100 \mathrm{fb}^{-1}\right)$ & $p p \rightarrow H_{S M} \rightarrow Z Z^{(*)} \rightarrow 4 \ell$ & $120 \lesssim m_{H_{S M}} \lesssim 700$ \\
& $p p \rightarrow H_{S M} \rightarrow \gamma \gamma$ & $80 \lesssim m_{H_{S M}} \lesssim 150$ \\
& $p p \rightarrow t \bar{t} H_{S M}, W H_{S M} ; H_{S M} \rightarrow \gamma \gamma$ & $80 \lesssim m_{H_{S M}} \lesssim 120-130$ \\
& $p p \rightarrow t \bar{t} H_{S M} ; H_{S M} \rightarrow b \bar{b}$ & $m_{H_{S M}} \lesssim 100-110$ \\
\hline NLC, $\left(500 \mathrm{GeV}, 50 \mathrm{fb}^{-1}\right)$ & $e^{+} e^{-} \rightarrow Z H_{S M}$ & $m_{H_{S M}} \lesssim 350$ \\
& $W W \rightarrow H_{S M}$ & $m_{H_{S M}} \lesssim 300$ \\
& $e^{+} e^{-} \rightarrow t \bar{t} H_{S M}$ & $m_{H_{S M}} \lesssim 120$ \\
\hline NLC, $\left(1 \mathrm{TeV}, 200 \mathrm{fb}^{-1}\right)$ & $e^{+} e^{-} \rightarrow Z H_{S M}$ & $m_{H_{S M}} \lesssim 800$ \\
& $W W \rightarrow H_{S M}$ & $m_{H_{S M}} \lesssim 700$ \\
\hline
\end{tabular}

\section{DISCLAIMER}

This report was prepared as an account of work sponsored by an agency of the United States Government. Neither the United States Government nor any agency thereof, nor any of their employees, makes any warranty, express or implied, or assumes any legal liability or responsibility for the accuracy, completeness, or usefulness of any information, apparatus, product, or process disclosed, or represents that its use would not infringe privately owned rights. Reference herein to any specific commercial product, process, or service by trade name, trademark, manufacturer, or otherwise does not necessarily constitute or imply its endorsement, recommendation, or favoring by the United States Government or any agency thereof. The views and opinions of authors expressed herein do not necessarily state or reflect those of the United States Government or any agency thereof. 
Table 3: MSSM Higgs boson discovery modes. All masses are specified in GeV. The ordered pair $\left(m_{A^{0}}, \tan \beta\right)$ fixes the MSSM Higgs sector masses and couplings. The parameter regime $m_{A^{0}} \leq 1000 \mathrm{GeV}$ and $1 \leq \tan \beta \leq 60$ is surveyed. If a range of $\tan \beta$ values is specified below, then the first (second) number in the range corresponds to the appropriate minimal (maximal) value of $m_{A^{0}}$. See text for further clarifications.

\begin{tabular}{|c|c|c|}
\hline $\operatorname{Machine}\left(\sqrt{s}, \int \mathcal{C} d t\right)$ & Mode & $\left(\boldsymbol{m}_{A^{\circ}}, \tan \boldsymbol{\beta}\right)$ Discovery Region \\
\hline LEP-I & $e^{+} e^{-} \rightarrow Z^{*} h^{0}, h^{0} A^{0}$ & $(\lesssim 25, \gtrsim 1)$ or $(\lesssim 45, \gtrsim 2)$ \\
\hline LEP-II $\left(176 \mathrm{GeV}, 500 \mathrm{pb}^{-1}\right)$ & $e^{+} e^{-} \rightarrow Z h^{0}, h^{0} A^{0}$ & $(\lessgtr 80, Z 1)$ or $(\$ 150, \lessgtr 4-1)$ \\
\hline LEP-II $\left(190 \mathrm{GeV}, 500 \mathrm{pb}^{-1}\right)$ & $e^{+} e^{-} \rightarrow Z h^{0}, h^{0} A^{0}$ & $(\lesssim 85, \gtrsim 1)$ or $(\gtrsim 85, \lesssim 5-1.5)$ \\
\hline $\mathrm{TeV}^{*}\left(2 \mathrm{TeV}, 30 \mathrm{fb}^{-1}\right)$ & $p \bar{p} \rightarrow W h^{0} ; h^{0} \rightarrow b \bar{b} ; 2 b$-tag & $(\geq 130-150, \geq 1)$ \\
\hline $\operatorname{DiTeV}\left(4 \mathrm{TeV}, 30 \mathrm{fb}^{-1}\right)$ & $p \bar{p} \rightarrow W h^{0} ; h^{0} \rightarrow b \bar{b} ; 2 b$-tag & $(\geq 130-150, \geq 1)$ \\
\hline LHC $\left(14 \mathrm{TeV}, 100 \mathrm{fb}^{-1}\right)$ & $\begin{array}{c}p p \rightarrow W h^{0}, t \bar{t} h^{0} ; h^{0} \rightarrow b \bar{b} ; 2,3 b \text {-tag } \\
p p \rightarrow t \bar{t} ; t \rightarrow H^{+} b ; 1 b \text {-tag } \\
p p \rightarrow H^{0} ; H^{0} \rightarrow Z Z^{(*)} \rightarrow 4 \ell \\
p p \rightarrow h^{0}, W h^{0}, t \bar{t} h^{0} ; h^{0} \rightarrow \gamma \gamma \\
p p \rightarrow b \bar{b} A^{0}, H^{0} ; A^{0}, H^{0} \rightarrow \tau^{+} \tau^{-} \\
p p \rightarrow b \bar{b} A^{0}, H^{0} ; A^{0}, H^{0} \rightarrow \mu^{+} \mu^{-} \\
p p \rightarrow b \bar{b} h^{0} ; h^{0} \rightarrow b \bar{b} ; 3 b \text {-tag } \\
p p \rightarrow b \bar{b} H^{0} ; H^{0} \rightarrow b \bar{b} ; 3 b \text {-tag } \\
p p \rightarrow b \bar{b} A^{0} ; A^{0} \rightarrow b \bar{b} ; 3 b \text {-tag }\end{array}$ & $\begin{array}{c}(\gtrsim 130-150, \geq 1) \\
(\lesssim 130, \geq 1) \\
(\lesssim 350, \lessgtr 10-2) \\
(\gtrsim 180, \geq 1) \\
(\gtrsim 100, \geq 5-50) \\
(\gtrsim 100, \geq 5-50) \\
(\lesssim 125, \geq 5-10) \\
(\gtrsim 125, \gtrsim 10-60) \\
(\lesssim 125, \gtrsim 5-10) \text { or }(\gtrsim 125, \geq 10-60)\end{array}$ \\
\hline $\operatorname{NLC}\left(500 \mathrm{GeV}, 50 \mathrm{fb}^{-1}\right)$ & $\begin{array}{c}e^{+} e^{-} \rightarrow Z h^{0} \\
W W \rightarrow h^{0} \\
e^{+} e^{-} \rightarrow h^{0} A^{0} \\
e^{+} e^{-} \rightarrow Z H^{0}, W W \rightarrow H^{0} \\
e^{+} e^{-} \rightarrow H^{0} A^{0} \\
e^{+} e^{-} \rightarrow H^{+} H^{-} \\
\end{array}$ & 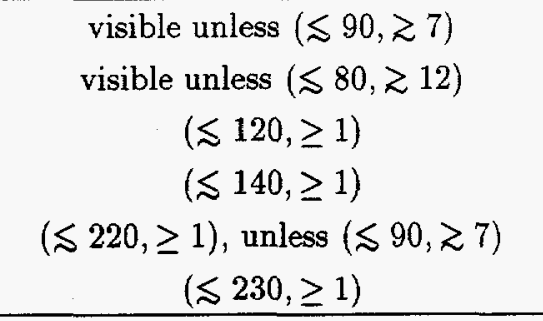 \\
\hline $\operatorname{NLC}\left(1 \mathrm{TeV}, 200 \mathrm{fb}^{-1}\right)$ & $\begin{array}{l}e^{+} e^{-} \rightarrow H^{0} A^{0} \\
e^{+} e^{-} \rightarrow H^{+} H^{-}\end{array}$ & $\begin{array}{c}(\lesssim 450, \geq 1), \text { unless }(\$ 90, \geq 7) \\
(\$ 450, \geq 1)\end{array}$ \\
\hline
\end{tabular}


at $\sqrt{s}=176 \mathrm{GeV}$ and $500 \mathrm{pb}^{-1}$ integrated luminosity, either $h^{0}$ or $A^{0}$ (or both) can be discovered via $e^{+} e^{-} \rightarrow$ $Z h^{0}, h^{0} A^{0}$ if $m_{A^{0}} \lesssim 80 \mathrm{GeV}$ and $\tan \beta \gtrsim 1$. A second discovery region also exists, beginning at $\tan \beta \leqslant 4$ (at $m_{A^{0}}=80 \mathrm{GeV}$ ) and ending at $m_{A^{0}} \lesssim 150 \mathrm{GeV}$ (at $\tan \beta=1$ ). If $\sqrt{s}$ is increased to $190 \mathrm{GeV}$, both discovery regions become larger; in particular, the latter region now begins at $\tan \beta \lesssim 5\left(\right.$ at $\left.m_{A^{0}}=85 \mathrm{GeV}\right)$ and ends at $\tan \beta \lesssim 1.5$ for the maximal value of $m_{A^{0}}$ considered. At the NLC-500, $h^{0}$ can be detected via $e^{+} e^{-} \rightarrow Z h^{0}$ unless $\tan \beta \geq 7$ and $m_{A^{0}} \lesssim 90 \mathrm{GeV}$. A similar discovery region exception appears for $W W \rightarrow h^{0}$. The reason such restrictions arise is that in the indicated region of parameter space, $H^{0}$ is SM-like in its couplings, while the $h^{0} Z Z$ and $h^{\circ} W^{+} W^{-}$couplings are very suppressed. The $Z H^{0} A^{0}$ coupling is likewise suppressed in the same parameter regime, which explains the other two discovery region exceptions listed in Table 3 .

\subsection{A Tour of Higgs Search Techniques at Future Colliders}

The goal of the Higgs search at present and future colliders is to examine the full mass range of the SM Higgs boson, and the full parameter space of the MSSM Higgs sector. The LHC can cover the entire range of SM Higgs boson masses from the upper limit of LEP-II $\left(m_{H_{S M}}=80\right.$ $95 \mathrm{GeV}$, depending on machine energy) to a Higgs mass of $700 \mathrm{GeV}$ (and beyond). The most difficult region for high luminosity hadron colliders is the intermediate-mass range $m_{H_{S M}}=80-130 \mathrm{GeV}$. The $\mathrm{LHC}$ detectors are being designed with the capability of fully covering the intermediate Higgs mass region. The $\mathrm{TeV}^{*}$ and DiTevatron may also have some discovery potential in this mass region. In contrast, the intermediate mass Higgs search at the NLC (which makes use of the same search techniques employed at LEP-II) is straightforward. At design luminosity, the NLC discovery reach is limited only by the center-of-mass energy of the machine.

In the search for the Higgs bosons of the MSSM, two objectives are: (i) the discovery of $h^{0}$ and (ii) the discovery of the non-minimal Higgs states $\left(H^{0}, A^{0}\right.$, and $H^{ \pm}$). Two theoretical results play a key role in the MSSM Higgs search. First, the mass of $h^{0}$ is bounded by $m_{h^{0}}^{\max } \simeq 120 \mathrm{GeV}$, as noted above. Second, if the properties of $h^{0}$ deviate significantly from the SM Higgs boson, then $m_{A^{0}} \lesssim \mathcal{O}\left(m_{Z}\right)$ and the $H^{0}$ and $H^{ \pm}$masses must lie in the intermediate Higgs mass region. As a result, experiments that are sensitive to the intermediate Higgs mass region have the potential for detecting at least one of the MSSM Higgs bosons over the entire MSSM Higgs parameter space (parametrized by $\tan \beta$ and $m_{A^{\circ}}$, as noted above). LEP-II does not have sufficient energy to cover the entire MSSM Higgs parameter space, since $m_{h^{0}}^{\max }$ lies above the LEP-II Higgs mass reach. The TeV*, DiTe- vatron and $\mathrm{LHC}$ all possess the capability of detecting Higgs bosons in the intermediate mass range, and consequently can probe regions of the MSSM Higgs parameter space not accessible to LEP-II. As of this writing, no set of experiments at any future hadron collider considered in this report can guarantee the discovery (or exclusion) of at least one MSSM Higgs boson for all values of $m_{A^{0}}$ and $\tan \beta$ not excluded by the LEP-II search. However, it may be possible to close this gap in the MSSM parameter space if sufficiently reliable $b$ quark identification in hadron collider events is possible. On the other hand, because of the relative simplicity of the NLC Higgs search in the intermediate mass region, the $\mathrm{NLC}$ is certain to discover at least one MSSM Higgs boson (either $h^{0}$ or $H^{0}$ ) if the supersymmetric approach is correct. If $h^{0}$ is discovered and proves to be SM-like in its properties, then one must be in the region of MSSM Higgs parameter space where the non-minimal Higgs states are rather heavy and approximately degenerate in mass. In this case, the LHC may not be capable of discovering any Higgs bosons beyond $h^{0}$, while $H^{0}, A^{0}$ and $H^{ \pm}$can be detected at the NLC only if its center-of-mass energy $\sqrt{s} \gtrsim 2 m_{A^{0}}$.

Below is a detailed description of the Higgs potential of present and future colliders. A Higgs boson is deemed observable if a $5 \sigma$-excess of events can be detected in a given search channel.

- LEP-I - The current lower bound on the SM Higgs mass is $64.5 \mathrm{GeV}$, and will increase by at most a few $\mathrm{GeV}$. The lower bounds on the MSSM Higgs masses are $m_{h^{\circ}}>44.5 \mathrm{GeV}, m_{A^{\circ}}>24.3 \mathrm{GeV}$ (for $\tan \beta>1$ ), and $m_{H^{ \pm}}>45 \mathrm{GeV}$.

- LEP-II - For $\sqrt{s}=176 \mathrm{GeV}$, the SM Higgs is accessible via $Z H_{S M}$ production up to $80 \mathrm{GeV}$ for an integrated luminosity of $500 \mathrm{pb}^{-1}$ [4]. For $\sqrt{s}=190 \mathrm{GeV}, m_{H_{S M}}$ values as high as $m_{Z}$ can be probed with $200 \mathrm{pb}^{-1}$ of data, using $b$-tagging in the region $m_{H_{S M}} \sim m_{Z}$. The reach for $\sqrt{s}=205 \mathrm{GeV}$ is about $105 \mathrm{GeV}$. In the MSSM, the same mass reaches apply to $h^{0}$ if $m_{A^{0}} \gtrsim m_{Z}$, since in this case $Z h^{0}$ is produced at about the same rate as $Z H_{S M}$. If $m_{A^{0}} \lesssim m_{Z}$, then the $h^{0} Z Z$ coupling (which controls the $Z h^{0}$ cross section) becomes suppressed while the $Z A^{0} h^{0}$ coupling becomes maximal. In the latter parameter region, $e^{+} e^{-} \rightarrow A^{0} h^{0}$ can be detected for all values of $m_{A^{\circ}} \lesssim \sqrt{s} / 2-10 \mathrm{GeV}$, assuming that $\tan \beta>1$. Since $m_{h^{\circ}} \lesssim 120 \mathrm{GeV}$, increasing the LEP-II energy to roughly $\sqrt{s}=220 \mathrm{GeV}$ while maintaining the luminosity would be be sufficient to guarantee the detection of at least one Higgs boson (via $Z h^{0}$ and/or $A^{0} h^{0}$ final states) over the entire MSSM Higgs parameter space $[4,5]$.

- Tevatron $\left(\sqrt{s}=2 \mathrm{TeV}, \mathcal{L}=10^{32} \mathrm{~cm}^{-2} \mathrm{~s}^{-1}\right.$ with the Main Injector) - The most promising mode for the SM Higgs is $W H_{S M}$ production, followed by $H_{S M} \rightarrow b \bar{b}[6]$. With $5 \mathrm{fb}^{-1}$ of integrated luminosity, this mode could potentially explore a Higgs mass region of $60-80 \mathrm{GeV}$, a 
region which will already have been covered by LEP II via the $Z H_{S M}$ process.

- $\mathrm{TeV}^{*}\left(\sqrt{s}=2 \mathrm{TeV}, \mathcal{L} \geq 10^{33} \mathrm{~cm}^{-2} \mathrm{~s}^{-1}\right)$ - The Higgs mass reach in the $W H_{S M}$ mode, with $H_{S M} \rightarrow b \vec{b}$, is extended over that of the Tevatron $[6,7]$. With $30 \mathrm{fb}^{-1}$ of integrated luminosity, a Higgs of mass $95 \mathrm{GeV}$ is potentially accessible, but the peak will not be separable from the $W Z, Z \rightarrow b \bar{b}$ background. The mode $(W, Z) H_{S M}$, followed by $H_{S M} \rightarrow \tau^{+} \tau^{-}$and $W, Z \rightarrow j j$ (where $j$ stands for a hadronic jet), is potentially viable for Higgs masses sufficiently far above the $Z$ mass [7]. With $30 \mathrm{fb}^{-1}$ of integrated luminosity, a Higgs in the mass range $110-120 \mathrm{GeV}$ may be detectable. However, the Higgs peak will be difficult to recognize on the slope of the much larger $Z j j, Z \rightarrow \tau^{+} \tau^{-}$background. Both of these modes are of particular significance for $h^{0}$. For $\tan \beta>1$ and $m_{A^{0}} \lesssim 1.5 m_{Z}$, the enhanced coupling of the $h^{0}$ to $b$ 's and $\tau$ 's makes it unobservable at the LHC via $h^{0} \rightarrow \gamma \gamma$ or $h^{0} \rightarrow Z Z^{*} \rightarrow 4 \ell$.

- DiTevatron $\left(\sqrt{s}=4 \mathrm{TeV}, \mathcal{L} \geq 10^{33} \mathrm{~cm}^{-2} \mathrm{~s}^{-1}\right)$ - For the SM Higgs, the "gold-plated" mode, $H_{S M} \rightarrow Z Z \rightarrow$ $4 \ell$, requires about $30 \mathrm{fb}^{-1}$ for the optimal Higgs mass in this mode, $m_{H_{S M}}=200 \mathrm{GeV}$ [6]. The $4 \ell$ mode is not useful for any of the MSSM Higgs bosons. The Higgs mass reach in the $W H_{S M}, H_{S M} \rightarrow b \bar{b}$ mode is only marginally better than at the $\mathrm{TeV}^{*}$, due to the increase in the topquark backgrounds relative to the signal $[6,7]$. The mode $(W, Z) H_{S M}$, with $H_{S M} \rightarrow \tau^{+} \tau^{-}$and $W, Z \rightarrow j j$, has less promise than at the $\mathrm{TeV}^{*}$ due to the relative increase in the background [7].

- $\operatorname{LHC}\left(\sqrt{\mathrm{s}}=14 \mathrm{TeV}, \mathcal{L}=10^{33}-10^{34} \mathrm{~cm}^{-2} \mathrm{~s}^{-1}\right)$ - For the SM Higgs boson, the "gold-plated" mode, $H_{S M} \rightarrow Z Z^{(*)} \rightarrow 4 \ell$, including the case where one $Z$ boson is virtual, covers the range of Higgs masses $130-700$ $\mathrm{GeV}$ and beyond with $100 \mathrm{fb}^{-1}[8,9]$. For $m_{H_{S M}}>700$ $\mathrm{GeV}$, the Higgs is no longer "weakly coupled", and search strategies become more complex - see Section 5.

The CMS detector is planning an exceptional electromagnetic calorimeter $\left(\mathrm{PbWO}_{4}\right.$ crystal) which will enable the decay mode $H_{S M} \rightarrow \gamma \gamma$ to cover the Higgs mass range $85-150 \mathrm{GeV}$ with $100 \mathrm{fb}^{-1}$ of integrated luminosity [9]. This range overlaps the reach of LEP II (with $\sqrt{s}=190 \mathrm{GeV}$ ) and the lower end of the range covered by the gold-plated mode. The ATLAS detector covers the range $110-140 \mathrm{GeV}$ with this mode [8]. Both CMS and ATLAS find that the modes $t \bar{t} H_{S M}$ and $W H_{S M}$, with $H_{S M} \rightarrow \gamma \gamma$, are viable in the mass range 80-120 $\mathrm{GeV}$ with $100 \mathrm{fb}^{-1}$ of integrated luminosity $[8,9]$. Since backgrounds are smaller, these modes do not require such excellent photon resolutions and jet-photon discrimination as does the inclusive $\gamma \gamma$ mode. CMS has also studied the production of the Higgs in association with two jets, followed by $H_{S M} \rightarrow \gamma \gamma$, and concludes that this mode covers the Higgs mass range $70-130 \mathrm{GeV}$ [9].

The modes $t \bar{t} H_{S M}$ and $W H$, with $H_{S M} \rightarrow b \bar{b}$, are potentially useful in the intermediate-mass region $[6,7]$. The reach with $100 \mathrm{fb}^{-1}$ of integrated luminosity is 100 $\mathrm{GeV}$, reduced to $80 \mathrm{GeV}$ with $30 \mathrm{fb}^{-1}$ [8]. Overall, ATLAS will cover the Higgs mass region $80-140 \mathrm{GeV}$ with $100 \mathrm{fb}^{-1}$ using a combination of $H_{S M} \rightarrow \gamma \gamma ; t \bar{t} H_{S M}$, $W H_{S M}$ with $H_{S M} \rightarrow \gamma \gamma$; and $t \bar{t} H_{S M}$ with $H_{S M} \rightarrow b \bar{b}$.

The main search mode for the lightest MSSM Higgs boson is $h^{0} \rightarrow \gamma \gamma$, which is viable when $m_{h^{0}}$ is near $m_{h^{0}}^{\max }[8,9,5,10,11]$. The processes $t \bar{t} h^{0}$ and $W h^{0}$, with $h^{0} \rightarrow b \bar{b}$, can potentially extend $h^{0}$ detection to the somewhat lower $m_{h}$ o values (corresponding to somewhat lower $m_{A^{\circ}}$ ) that would assure that at least one MSSM Higgs boson can be detected over all of the MSSM parameter space [6]. Adapting the ATLAS study for the SM Higgs to $h^{0}$ suggests that the required sensitivity could be achieved in the $t \bar{t} h^{0}$ mode. Due to the large top-quark background, the $W h^{0}$ with $h^{0} \rightarrow b \bar{b}$ mode at the LHC has little advantage over this mode at the $\mathrm{TeV}^{*}$ or the DiTevatron for the same integrated luminosity.

The other MSSM Higgs bosons are generally more elusive. There are small regions of parameter space in which $H^{0}$ can be observed decaying to $\gamma \gamma$ or $Z Z^{(*)}[8,9]$. The possibility exists that the charged Higgs can be discovered in top decays $[8,9]$. For $m_{A^{0}}>2 m_{Z}$ and large $\tan \beta, H^{0}$ and $A^{0}$ can have sufficiently enhanced $b$ quark couplings that they would be observed when produced in association with $b \bar{b}$ and decaying via $H^{0}, A^{0} \rightarrow \tau^{+} \tau^{-}$ $[8,9,11,6]$. For very enhanced couplings of the Higgs bosons to $b$ quarks (which occurs for very large $\tan \beta$ ), the modes $b \bar{b}\left(h^{0}, H^{0}, A^{0}\right)$, with $h^{0}, H^{0}, A^{0} \rightarrow b \bar{b}$, and $\bar{t} b H^{+}$, with $H^{+} \rightarrow t \bar{b}$, are potentially viable [6].

Let us suppose that the modes involving $b$-tagging (especially $t \bar{t} h^{0}$ production with $h^{0} \rightarrow b \bar{b}$ ) eventually prove to be viable. Then, the LHC Higgs search using the $b$-tagging modes in combination with the $4 \ell$ and $\gamma \gamma$ final state modes should be capable of discovering at least one of the MSSM Higgs bosons (or in absence of a discovery rule out the MSSM) [6]. For much of parameter space only the $h^{0}$ will be detectable. Detection of all the MSSM Higgs bosons at the LHC is only possible for very small regions of parameter space at moderate values of $m_{A^{0}}$.

- $e^{+} e^{-}$linear collider $(\sqrt{s}=500 \mathrm{GeV}$ to $1 \mathrm{TeV}, \mathcal{L} \geq$ $5 \times 10^{33} \mathrm{~cm}^{-2} \mathrm{~s}^{-1}$ ) - At $\sqrt{s}=500 \mathrm{GeV}$ with an integrated luminosity of $50 \mathrm{fb}^{-1}$, the SM Higgs boson is observable via the $Z H_{S M}$ process up to $m_{H_{S M}}=350$ $\mathrm{GeV}$ [12]. Employing the same process, a Higgs boson with $m_{H_{S M}}=130 \mathrm{GeV}(200 \mathrm{GeV})$ would be discovered with $1 \mathrm{fb}^{-1}\left(10 \mathrm{fb}^{-1}\right)$ of data. Other Higgs production mechanisms are also useful. With $50 \mathrm{fb}^{-1}$ of data, the $W W$-fusion process is observable up to $m_{H_{S M}}=300$ $\mathrm{GeV}[12,13,14]$. The $t \bar{t} H_{S M}$ process is accessible for $m_{H_{S M}} \lesssim 120 \mathrm{GeV}$, thereby allowing a direct determination of the $t \bar{t} H_{S M}$ Yukawa coupling [13]. The $\gamma \gamma$ collider mode of operation does not extend the reach of the ma- 
chine for the SM Higgs, but does allow a measurement of the $H_{S M} \rightarrow \gamma \gamma$ partial width up to $m_{H_{S M}}=300$ $\mathrm{GeV}[13]$.

In the MSSM, for $m_{A^{0}} \lesssim m_{Z}$, the $h^{0}$ and $A^{0}$ will be detected in the $h^{0} A^{0}$ mode, the $H^{0}$ will be found via $Z H^{0}$ and $W W$-fusion production, and $H^{+} H^{-}$pair production will be kinematically allowed and easily observable $[15,13,14]$. At higher values of the parameter $m_{A^{0}}$, the lightest Higgs boson is accessible in both the $Z h^{0}$ and $W W$-fusion modes. However, the search for the heavier Higgs boson states is limited by machine energy. The mode $H^{0} A^{0}$ is observable up to $m_{H^{\circ}} \sim m_{A^{0}} \sim 220$ $\mathrm{GeV}$, and $H^{+} H^{-}$can be detected up to $m_{H^{ \pm}}=230$ $\mathrm{GeV}[15,13,14]$. The $\gamma \gamma$ collider mode could potentially extend the reach for $H^{0}$ and $A^{0}$ up to $400 \mathrm{GeV}$ if $\tan \beta$ is not large [5].

At $\sqrt{s}=1 \mathrm{TeV}$ with an integrated luminosity of $200 \mathrm{fb}^{-1}$, the SM Higgs boson can be detected via the $W W$-fusion process up to $m_{H_{S M}}=700 \mathrm{GeV}[13,14]$. In the MSSM, $H^{0} A^{0}$ and $H^{+} H^{-}$detection would be extended to $m_{H^{0}} \sim m_{A^{\circ}} \sim m_{H^{ \pm}} \sim 450 \mathrm{GeV}[5,15,13,14]$. Measurements of cross sections, branching ratios, and angular distributions of the Higgs events will determine model parameters and test the underlying theory of the scalar sector [13].

- Influence of MSSM Higgs decays into supersymmetric particle final states - For some MSSM parameter choices, the $h^{0}$ can decay primarily to invisible modes, including a pair of the lightest supersymmetric neutralinos or a pair of invisibly decaying sneutrinos [6]. The $h^{0}$ would still be easily discovered at $e^{+} e^{-}$colliders in the $Z h^{0}$ mode using missing-mass techniques [13]. At hadron colliders, the $W h^{0}$ and $t \bar{t} h^{0}$ modes may be detectable via large missing energy and lepton plus invisible energy signals, but determination of $m_{h}$ o would be difficult [6]. The $H^{0}, A^{0}$, and $H^{ \pm}$decays can be dominated by chargino and neutralino pair final states and/or slepton pair final states $[2,5,10,6]$. Such modes can either decrease or increase the chances of detecting these heavier MSSM Higgs bosons at a hadron collider, depending upon detailed MSSM parameter choices $[5,10,6]$. At $e^{+} e^{-}$colliders $H^{0}, A^{0}$ and $H^{ \pm}$detection up to the earlier quoted (largely kinematical) limits should in general remain possible.

- Hadron collider beyond the LHC - It could provide increased event rates and more overlap of discovery modes for the SM Higgs boson. However, its primary impact would be to extend sensitivity to high mass signals associated with strongly-coupled electroweak symmetry breaking scenarios (see Section 5). In the case of the MSSM Higgs bosons, the increased event rates would significantly extend the overlap of the various discovery modes, expanding the parameter regions where several and perhaps all of the MSSM Higgs bosons could be observed. - $e^{+} e^{-}$collider beyond $\sqrt{s}=1 \mathrm{TeV}$ - Such an exten- sion in energy is not required for detecting and studying a weakly-coupled SM Higgs bosons, but could be very important for a strongly-coupled electroweak-symmetrybreaking scenario. Detection of $H^{0} A^{0}$ and $H^{+} H^{-}$production in the MSSM model would be extended to higher masses.

\subsection{Precision Measurements of Higgs Properties}

Detailed studies of the properties of Higgs bosons can be carried out at $e^{+} e^{-}$colliders. The measurements of cross sections, branching ratios, and angular distributions of Higgs events will determine model parameters and test the underlying theory of the scalar sector. In this discussion, $h$ denotes the lightest CP-even neutral Higgs. It may be $H_{S M}$ or $h^{0}$ (of the MSSM), or perhaps a scalar arising from a completely different model. Once discovered, determining the identity of $h$ is precisely what one hopes to be able to address based on the considerations presented here. Below we list some examples of the measurements that can be made at $\sqrt{s}=500 \mathrm{GeV}$ with an integrated luminosity of $50 \mathrm{fb}^{-1}$, assuming that the Higgs mass is less than $350 \mathrm{GeV}$ and that the process $e^{+} e^{-} \rightarrow Z h$ occurs at approximately the Standard Model rate.

The single event Higgs mass resolution using $e^{+} e^{-} \rightarrow$ $Z h$ is approximately $4 \mathrm{GeV}$ [14]. The number of Higgs events provided by an integrated luminosity of $50 \mathrm{fb}^{-1}$ will then allow the determination of the central value of the Higgs mass with a statistical accuracy of \pm 180 $\mathrm{MeV}$. Since most Standard Model parameters only have logarithmic dependence on the Higgs mass, this accuracy is far greater than what is needed to check the selfconsistency of the Standard Model. One could use this mass determination to make accurate predictions for the Higgs production cross sections and branching fractions.

For a SM Higgs boson, signals can be selected with small backgrounds and one can easily measure the angular distributions of the Higgs production direction in the process $e^{+} e^{-} \rightarrow Z h$ and the directions of the outgoing fermions in the $Z^{0}$ rest frame [14], and verify the expectations for a CP-even Higgs boson [16]. For a more general Higgs eigenstate, since only the CP-even part couples at tree-level to $Z Z$, these measurements would still agree with the CP-even predictions unless the Higgs is primarily (or purely) CP-odd, in which case the cross section will be much smaller and the event rate inadequate to easily verify the mixed-CP or CP-odd distribution forms. Analysis of photon polarization asymmetries in $\gamma \gamma \rightarrow h$ production rates, and of angular correlations among secondary decay products arising from primary $h \rightarrow \tau \tau$ or $h \rightarrow t \bar{t}$ final state decays in $Z h$ production, can both provide much better sensitivity to a CP-odd Higgs component in many cases [17].

By choosing a decay channel whose reconstruction is 
independent of the Higgs mass and decay mode, such as $e^{+} e^{-} \rightarrow Z h$, where $Z \rightarrow \ell^{+} \ell^{-}$, one can measure the total cross section for Higgs production. For an integrated luminosity of $50 \mathrm{fb}^{-1}$, this can be determined with a statistical accuracy of better than $10 \%$ [14]. This is one possible technique for measuring the $h Z Z$ coupling.

A specific study [18] has been conducted to consider the experimental problems of determining the production cross section $\sigma$ times the branching fractions of a discovered Higgs boson into all of its possible (Standard Model) final states. The resulting expected statistical precisions are strong functions of the mass of the Higgs, as the branching fractions change dramatically in the mass range $100 \mathrm{GeV}<m_{h}<180 \mathrm{GeV}$. For a Standard Model Higgs of mass $120 \mathrm{GeV}$, it is found that statistical precisions of $7 \%, 14 \%, 39 \%$, and $48 \%$ can be achieved for $\sigma \times B R(h \rightarrow b \bar{b}), \sigma \times B R\left(h \rightarrow \tau^{+} \tau^{-}\right)$, $\sigma \times B R(h \rightarrow c \bar{c}+g g)$, and $\sigma \times B R\left(h \rightarrow W W^{*}\right)$ respectively.

Reasonably precise measurements of certain $\sigma \times B R$ combinations will also be possible at hadron colliders. For example, at the LHC a good determination of $\sigma(g g \rightarrow$ $h) \times B R(h \rightarrow \gamma \gamma)$ should be possible for a SM-like Higgs in the 80 to $150 \mathrm{GeV}$ mass range, and of $\sigma(g g \rightarrow h) \times$ $B R(h \rightarrow Z Z)$ for $m_{h}>130 \mathrm{GeV}$.

\section{Low Energy Supersymmetry: Implications of Models}

\subsection{Elements of Minimal Low-Energy Supersymmetry}

A truly fundamental theory of particles and their interactions must account for the large hierarchy of energy scales inherent in the theory-from the scale of electroweak symmetry breaking [characterized by $\left(\sqrt{2} G_{F}\right)^{-1 / 2}=$ $246 \mathrm{GeV}]$ up to energies as large as the Planck scale (of order $10^{19} \mathrm{GeV}$ ). Supersymmetry (SUSY) provides a mechanism for stabilizing the large hierarchy of scales, and thus is a natural framework for addressing such problems. The simplest example of such an approach is the minimal supersymmetric extension of the Standard Model (MSSM). The MSSM possesses a new class of particles-supersymmetric partners of the quarks, leptons, gauge and Higgs bosons of the Standard Model. A particle and its supersymmetric partner would be degenerate in mass if supersymmetry were an exact symmetry of nature. Since this degeneracy is not realized in nature, supersymmetry must be broken. But, if the breaking is "soft" (with supersymmetry-breaking masses of order $1 \mathrm{TeV}$ or less), then the stability of the hierarchy can be successfully maintained.

Unification of gauge couplings provides an intriguing hint for an underlying supersymmetric theory of particle interactions. If one extrapolates the $\mathrm{SU}(3) \times \mathrm{SU}(2) \times \mathrm{U}(1)$ gauge couplings from their low-energy values measured at LEP to very large energy scales, one finds that the three couplings meet at approximately the same energy scale if the renormalization group equations (RGE's) of the MSSM are used [19]. In contrast, if Standard Model RGE's are used, unification of gauge couplings fails by more then seven standard deviations! As a result, it is possible to construct a model of particle interactions such that: (i) the strong and electroweak forces are unified at very large energies (at a scale $M_{X} \sim 2 \times 10^{16} \mathrm{GeV}$, which is not too far from the Planck scale, $M_{P}=$ $10^{19} \mathrm{GeV}$ ); (ii) the couplings of all particles with nontrivial $\mathrm{SU}(3) \times \mathrm{SU}(2) \times \mathrm{U}(1)$ quantum numbers are perturbative in strength at all energies below the Planck scale; and (iii) the stability of the large hierarchy between the electroweak and grand unified energy scales is guaranteed by the underlying supersymmetry. In this approach, the origin of the electroweak scale is tied to the scale of supersymmetry breaking. In particular, in the models considered later in this section, electroweak symmetry breaking is generated through radiative corrections. Briefly stated, the squared Higgs mass parameter is positive at the high energy scale, $M_{X}$. However, due to the one-loop radiative effects generated by the large top quark Higgs Yukawa coupling (i.e., as a consequence of the large top quark mass), the squared Higgs mass parameter is driven negative in its evolution from $M_{X}$ down to the low energy electroweak scale [20]. These effects trigger electroweak symmetry breaking at the proper mass scale (of order $v=246 \mathrm{GeV}$ ) and establish the large hierarchy of scales between $M_{X}$ and $m_{Z}$.

Although the electroweak symmetry breaking scale has now been related to the supersymmetry breaking scale, one has not yet explained where the supersymmetry-breaking masses come from. As noted above, these masses must be of order $1 \mathrm{TeV}$ or less in order to generate the electroweak symmetry breaking scale without an excessive ("unnatural") fine-tuning of model parameters. The fundamental origin of supersymmetry breaking is one of the most important unsolved problems of supersymmetry theory. Nevertheless, one can take a purely phenomenological approach and parameterize the MSSM in terms of a general set of a priori unknown supersymmetry breaking parameters. Ultimately, these parameters would be measured in experiment once supersymmetric particles are discovered. A detailed knowledge of these parameters could provide important clues as to their origins.

A brief synopsis of the parameters of the MSSM is now presented. The SUSY-conserving part of the MSSM is fixed by the three gauge coupling constants of the Standard Model, the Higgs-fermion Yukawa couplings (denoted by $h_{U}, h_{D}$ and $h_{E}$ below), and one new supersymmetric Higgs mass parameter $\mu$. The particle content of the MSSM consists of three families of quarks and leptons and their scalar partners; the vector gauge bosons and 
their fermionic gaugino partners corresponding to the gauge group $\mathrm{SU}(3) \times \mathrm{SU}(2) \times \mathrm{U}(1)$; and two $\mathrm{SU}(2)$-doublet Higgs scalars and their fermionic higgsino partners. The introduction of a second Higgs doublet is necessary in order for the gauge anomalies associated with the higgsinos to cancel, and to give masses to both "up-type" quarks and "down-type" quarks and charged leptons (the latter cannot be done with one Higgs scalar without violating SUSY). As a result, the Higgs sector of the MSSM is a two-Higgs doublet model, whose phenomenology was discussed in great detail in Section 2. The colorless gauginos and higgsinos with the same electric charge can mix. The resulting mass eigenstates are called charginos and neutralinos, with corresponding electric charges \pm 1 and 0 , respectively. With the particle content and the Higgsfermion Yukawa couplings fixed as described above, all SUSY-preserving interactions (which correspond to all the dimensionless couplings of the model) are determined by the $\mathrm{SU}(3) \times \mathrm{SU}(2) \times \mathrm{U}(1)$ gauge invariance and/or by the supersymmetry.

However, in contrast to the Standard Model, the Higgs-fermion Yukawa couplings do not exhaust all possible scalar-fermion interactions. The MSSM possesses new scalars-squarks and sleptons-which carry baryon number $(B)$ and lepton number $(L)$, respectively. Consequently, it is possible to write down additional scalarfermion interaction terms which violate either $B$ or $L$ (but preserve supersymmetry). In the MSSM, one simply assumes that these terms are not present. This can be implemented with a discrete symmetry, called $R$-parity which assigns $(-1)^{2 S+3(B-L)}$ to particles of spin $S$ [i.e., +1 to all Standard Model particles and -1 to their supersymmetric partners], and is multiplicatively conserved by all interactions of the model. $R$-parity conservation guarantees the existence of a stable lightest supersymmetric particle (LSP), which is an excellent candidate for dark matter. Although there are some strong phenomenological constraints on $R$-parity violating interactions, it is possible to construct $R$-violating models which are not inconsistent with present data. Such models lie outside the scope of the MSSM, and will be briefly mentioned at the end of this section.

The SUSY-breaking part of the MSSM is where most of the new parameters of the MSSM lie. In addition to breaking the mass degeneracy between particles and their supersymmetric partners, the SUSY-breaking terms are required in order to break the $\mathrm{SU}(2) \times \mathrm{U}(1)$ electroweak gauge symmetry. The Higgs potential, with soft-SUSY breaking mass terms included, can have a non-trivial minimum. Both neutral Higgs fields acquire vacuum expectation values; this introduces an important new parameter, $\tan \beta \equiv v_{2} / v_{1}$, where $v_{i} \equiv\left\langle H_{i}^{0}\right\rangle$. Note that after electroweak symmetry breaking, the Yukawa coupling matrices are proportional to the corresponding quark and lepton mass matrices. (In the limit where only third generation Yukawa couplings are considered, $h_{\tau}=\sqrt{2} m_{\tau} /(v \cos \beta), h_{b}=\sqrt{2} m_{b} /(v \cos \beta)$, and $h_{t}=$ $\sqrt{2} m_{t} /(v \sin \beta)$, where $v=\left(v_{1}^{2}+v_{2}^{2}\right)^{1 / 2}=246 \mathrm{GeV}$.)

Supersymmetric breaking parameters are not completely arbitrary, since they must preserve the stability of the hierarchy discussed above. Such terms, called "soft", were classified in ref. [21], and consist of masses for scalars (but not their fermionic partners), masses for the gauginos, and trilinear scalar couplings. If there were no reason for the scalar masses and trilinear couplings to be diagonal in the same basis as the Yukawa couplings, then there would be a huge number $(>100)$ of possible new SUSY-breaking parameters. In models based on supergravity [22], a dramatic simplification occurs. SUSY is presumed to be broken in a "hidden sector" of particles which have only gravitational interactions with the "visible sector" of quarks, leptons, gauge and Higgs bosons, and their supersymmetric partners. The SUSY breaking is then transmitted to the visible sector by gravitational interactions, which results in the softSUSY breaking terms of the MSSM, characterized by a mass scale $M_{\text {SUSY }} \lesssim \mathcal{O}(1 \mathrm{TeV})$. Moreover, in the minimal approach, the soft-SUSY breaking terms are taken to be flavor independent and can be summarized in terms of a few universal parameters, regardless of the unknown physics in the hidden sector. The SUSY-breaking scalar interactions which arise take the form

$$
\begin{aligned}
\mathcal{L}_{\text {soft }}= & m_{0}^{2}\left[\left|H_{1}\right|^{2}+\left|H_{2}\right|^{2}\right. \\
& \left.+\sum_{\text {families }}\left(\left|Q_{L}\right|^{2}+\left|U_{R}\right|^{2}+\left|D_{R}\right|^{2}+\left|L_{L}\right|^{2}+\left|E_{R}\right|^{2}\right)\right] \\
& +A_{0}\left(h_{U} Q_{L} H_{2} U_{R}+h_{D} Q_{L} H_{1} D_{R}+h_{E} L_{L} H_{1} E_{R}\right) \\
& +B \mu H_{1} H_{2}+\text { h.c. }
\end{aligned}
$$

This simple form of the SUSY-breaking Lagrangian, which depends on three new SUSY-breaking parameters: $m_{0}^{2}, A$, and $B$, holds at a mass scale close to the Planck mass. In addition, three soft-SUSY-breaking Majorana mass terms can be.introduced for the fermionic gaugino partners of the $\mathrm{SU}(3) \times \mathrm{SU}(2) \times \mathrm{U}(1)$ gauge bosons, respectively. If one adds the additional assumption of minimal grand unification at a high energy scale $M_{X}$, then one finds a further reduction of parameters. Both the gauge couplings and the gaugino masses unify at $M_{X}$. There may be additional relations among the Yukawa couplings, although these depend on the details of the superheavy particle spectrum and their interactions associated with the grand unified gauge group. The supersymmetry model parameters can then be taken to be: (i) the common mass parameter $m_{1 / 2}$ for the gauginos, (ii) a common scalar mass parameter $m_{0}$, (iii) a common trilinear scalar coupling parameter $A_{0}$, (iv) the ratio of the Higgs vacuum expectation values, $\tan \beta$, and $(\mathrm{v})$ the sign of $\mu$. (The parameters $|\mu|$ and $B$ can be eliminated 
in favor of $\tan \beta$ and $m_{Z}$ by minimizing the scalar potential.) Here $m_{1 / 2}, m_{0}$, and $A_{0}$ are all typically of order $M_{\text {SUSY. }}$.

To find the Lagrangian relevant for physics at the electroweak scale, one must evolve the parameters of the model according to their renormalization group equations from $M_{X}$ down to $m_{Z}$. This splits the gaugino masses and the various scalar masses at low energies. The most striking result of this running is that radiative corrections due to the large top Yukawa coupling push the squared mass of $H_{2}$ negative at scales far below $M_{X}$, resulting in electroweak symmetry breaking [20]. This is a remarkable effect, connected with the large top quark mass, and exhibits the intimate relation between lowenergy supersymmetry and electroweak symmetry breaking. The large top quark mass also has an impact on a number of other MSSM parameters via the effects of the large top-quark Yukawa coupling in the RGE's. For example, one finds that the squared masses of the top squarks and left-handed bottom squark are reduced relative to other squark masses, while the mass of the lightest CP-even neutral Higgs boson may be substantially increased above $m_{Z}$.

\subsection{Features of the Superpartner Spectrum}

With the masses of all the superpartners determined only by the parameters $\left(m_{1 / 2}, m_{0}, A_{0}, \tan \beta, \operatorname{sign} \mu\right)$, the spectrum is highly constrained. With a universal $m_{0}$, squarks (or sleptons) with the same gauge quantum numbers will remain degenerate even at low energies, unless they have large Yukawa couplings. This degeneracy is a success of the model, since it automatically maintains the suppression of FCNCs in low-energy physics. (This remains true even if one relaxes the usual minimal assumptions by introducing a larger gauge group at some intermediate scale or allowing the gaugino mass parameters to be independent.) So the superpartner spectrum will contain seven distinct groups of degenerate scalar states $\left(\tilde{u}_{L}, \tilde{c}_{L}\right) ;\left(\tilde{d}_{L}, \tilde{s}_{L}\right) ;\left(\tilde{u}_{R}, \tilde{c}_{R}\right) ;\left(\tilde{d}_{R}, \tilde{s}_{R}, \tilde{b}_{R}\right)$; $\left(\tilde{e}_{L}, \tilde{\mu}_{L}, \tilde{\tau}_{L}\right) ;\left(\tilde{\nu}_{e}, \tilde{\nu}_{\mu}, \tilde{\nu}_{\tau}\right) ;\left(\tilde{e}_{R}, \tilde{\mu}_{R}, \tilde{\tau}_{R}\right)$. If $\tan \beta \gg 1$, then the bottom and tau Yukawa couplings are non-negligible, so that $\tilde{b}_{R}, \tilde{\tau}_{L}, \tilde{\tau}_{R}$, and $\tilde{\nu}_{\tau}$ need not be degenerate with their colleagues. With the minimal assumptions, one finds for the physical squark and slepton masses of the first two families approximately

$$
\begin{aligned}
m_{\tilde{e}_{R}}^{2} & =m_{0}^{2}+0.15 m_{1 / 2}^{2}-0.23 m_{Z}^{2} \cos 2 \beta ; \\
m_{\tilde{e}_{L}}^{2} & =m_{0}^{2}+0.52 m_{1 / 2}^{2}-0.27 m_{Z}^{2} \cos 2 \beta ; \\
m_{\tilde{\nu}}^{2} & =m_{0}^{2}+0.52 m_{1 / 2}^{2}+0.50 m_{Z}^{2} \cos 2 \beta ; \\
m_{\tilde{q}}^{2} & =m_{0}^{2}+(4.5 \text { to } 6.5) m_{1 / 2}^{2} .
\end{aligned}
$$

Eq. (3.2d) holds for first and second generation squark mass. The range in the coefficient of $m_{1 / 2}^{2}$ is partly due to the uncertainty in $\alpha_{3}$; moreover, this coefficient is smaller for larger squark masses. Eqs. (3.2) imply $m_{\tilde{q}}>m_{\tilde{e}_{L}}>m_{\tilde{e}_{R}}$. At least one of the top squark mass eigenstates and usually a bottom squark are below the main squark band implied by (3.2d), because of radiative effects from the top Yukawa coupling. This ordering of squark masses should lead to enhanced production of third generation squarks at hadron colliders, and to enhanced branching ratios of gluinos, charginos and neutralinos into third generation quarks.

In the minimal approach, the physical gluino mass is related to $m_{1 / 2}$ by

$$
M_{\tilde{g}}=(2.2 \text { to } 3.5) m_{1 / 2}
$$

Therefore, the squarks whose corresponding Higgs-quark Yukawa couplings are small cannot be much lighter than the gluino. When correlations among parameters are taken into account, one finds $m_{\tilde{q}}>0.8 M_{\tilde{g}}$. With $m_{t} \simeq$ $175 \mathrm{GeV}$, radiative electroweak breaking requires that $|\mu|$ be large compared to the electroweak gaugino masses. The lighter two neutralinos and the lighter chargino will then be gaugino-like, and the heavier neutralinos and chargino will only rarely be produced in the decays of squarks and gluinos. This also implies that the heavier Higgs mass eigenstates $A, H^{0}$ and $H^{ \pm}$will be quite heavy and almost degenerate; they will usually also be able to decay into charginos and neutralinos. The lightest Higgs scalar, $h^{0}$, will have couplings close to those of the Standard Model Higgs boson. There is an upper bound on the $h^{0}$ mass of about $120 \mathrm{GeV}$ in the MSSM. A similar $h^{0}$ mass bound of about $150 \mathrm{GeV}$ is found in a completely general class of low-energy supersymmetric models [23] (assuming all model couplings are perturbative up to $M_{X}$ ).

\subsection{Beyond the Minimal Planck Scale Assumptions}

Many model builders have begun to explore the consequences of relaxing the minimal assumptions outlined above. For example, the boundary condition of universal scalar masses at $M_{X}$ can be modified by non-trivial string effects, threshold effects associated with the grand unified model, evolution effects between $M_{X}$ and $M_{P}$, or additional contributions arising from the effects of a larger gauge symmetry group which is broken at a scale above $M_{X}[24]$. Generally, small or moderate changes of the boundary conditions at $M_{X}$ will only be important near the border of the allowed region of parameter space. For example, if the mass squared of $H_{1}$ is larger than that of $H_{2}$ at the scale $M_{X}, \tan \beta$ of order $m_{t} / m_{b}$ or larger becomes possible without strong fine-tuning of soft breaking parameters. Non-universal scalar masses may also reduce $|\mu|$, possibly allowing for light higgsinos. 
One can even entertain the possibility of large nonuniversalities among sfermions with the same electroweak quantum numbers. One often finds the statement in the literature that near-degeneracy of sfermion masses at the scale $M_{X}$ is required by bounds on FCNC. This statement is not entirely correct; the potentially most dangerous gluino loop contributions will always vanish if quark and squark mass matrices are aligned [25], i.e. can be diagonalized simultaneously. This is true independent of the (differences between) squark masses. The same is also true for neutralino loops. In such models only chargino loops contribute to FCNC. Notice that in general these do not constrain masses of $S U(2)$ singlet squarks. The most sensitive bound comes from $K^{0}-\overline{K^{0}}$ mixing. Demanding that the chargino loop contribution to $\Delta m_{K}$ be smaller than the experimental value of $3.5 \times 10^{-15} \mathrm{GeV}$ gives

$$
\left|\delta_{\tilde{u} \tilde{c}}\right| \leq 0.8 \mathrm{TeV}^{-1}\left(1+6 m_{1 / 2}^{2} / m_{0}^{2}\right) \sqrt{4.3 m_{1 / 2}^{2}+0.4 m_{0}^{2}}
$$

where $m_{0}^{2}$ is the average and $\delta_{\tilde{u} \tilde{c}} \cdot m_{0}^{2}$ the difference between the squared $\tilde{u}_{L}$ and $\tilde{c}_{L}$ masses at scale $M_{X}$. We see that $\delta_{\tilde{u} \tilde{c}} \simeq 1$ is allowed even for $m_{0}=m_{1 / 2}=100$ $\mathrm{GeV}$. Highly non-degenerate squarks are therefore allowed if quark and squark mass matrices are (approximately) aligned. This might make squark hunting at hadron colliders difficult, since the signal from any one squark state might be too weak to be visible above backgrounds.

One should also consider extensions of the MSSM that go beyond simple modifications of the MSSM boundary conditions at the scale $M_{X}$. The "Next-to-Minimal" Supersymmetric extension of the Standard Model introduces a gauge-singlet Higgs chiral supermultiplet in addition to the usual MSSM particles. This variation does not seem to have a major effect on the spectrum of the MSSM particles [26], but it does impact on discovery channels for the Higgs and neutralinos. In particular, one may have a light Higgs scalar with reduced couplings to the $Z$ boson. One can also contemplate low-energy supersymmetric models in which $R$-parity conservation is absent. For example, $R$-parity could be broken spontaneously by a sneutrino vacuum expectation value $\langle\tilde{\nu}\rangle \neq 0$. One can also replace $R$-parity by an alternative discrete symmetry [27] which allows either baryon number or lepton number violation (but not both!) among the renormalizable interactions. The phenomenology of $R$-parity violating models is quite different from that of the MSSM. This is mainly due to the fact that lightest supersymmetric particle is no longer stable, but can decay via an $R$-parity violating interaction. No systematic study of $R$-parity violating models in the context of supergravity and superunified theories has yet been undertaken.

\section{Low Energy Supersymmetry: Phenomenology}

If supersymmetry is associated with the origin of the electroweak symmetry breaking scale, then low-energy supersymmetry must exist at a scale of order $1 \mathrm{TeV}$ or below. That is, supersymmetry must be discovered at either upgrades of existing colliders or at the LHC. Due to its mass reach, the LHC is the definitive machine for discovering or excluding low-energy supersymmetry. Section 4.1 summarizes the supersymmetry mass discovery potential for hadron colliders. A variety of signatures are explored. The conventional models of low-energy supersymmetry (which are $R$-parity conserving) possess a stable lightest supersymmetric particle (LSP) which is electrically (and color) neutral and therefore interacts very weakly in matter. It follows that the LSP behaves like a heavy neutrino which does not interact in collider detectors. Its presence would be signaled by excess missing transverse energy (which could not be attributed to neutrinos). Many of the supersymmetry searches rely on the missing energy signature as an indication of new physics beyond the Standard Model. Multi-leptonic signatures also play an important role in supersymmetry searches at hadron colliders. (Such signals can also be exploited in the search for $R$-parity-violating low-energy supersymmetry.)

Suppose that a signal is observed in one of the expected channels. This would not be a confirmation of low-energy supersymmetry, unless there is confirming evidence from other expected signatures. This presents a formidable challenge to experimenters at the LHC. Can they prove that a set of signatures of new physics is lowenergy supersymmetry? Can they extract parameters of the supersymmetric models with any precision and test the details of the theory? These are questions that have only recently attracted seriously study. It is in this context that a future $e^{+} e^{-}$collider can be invaluable. As shown in Section 4.2. if the lightest supersymmetric particles were produced at LEP-II or the NLC, precision measurement could begin to map out in detail the parameter space of the supersymmetric model. In particular, beam polarization at the NLC provides an invaluable tool for studying the relation between chirality and the properties of supersymmetric particles. For example, one can begin to prove that there is a correlation between the left and right-handed electrons and their slepton partners as expected in supersymmetry. The elucidation of the details of the light chargino and neutralino spectrum at the NLC could also play a pivotal role in untangling complex decay chains of heavier supersymmetric particles produced at the LHC.

\subsection{Supersymmetry at Hadron Colliders}

The detection of supersymmetry at a hadron colliter 
Table 4: Discovery reach of various options of future hadron colliders. The numbers are subject to $\pm 15 \%$ ambiguity. Also, the clean trilepton signals are sensitive to other model parameters; we show representative ranges from ref. [31] where $|\mu|$ is typically much larger than the soft-breaking electroweak gaugino masses. For $\mu>0$, the leptonic decay of $\widetilde{\chi}_{2}^{0}$ may be strongly suppressed so that $3 \ell$ signals may not be observable even if charginos are just above the LEP bound $\left(m_{\tilde{g}} \sim 150 \mathrm{GeV}\right)$.

\begin{tabular}{|c|cccccc|}
\hline & Tevatron I & Tevatron II & Main Injector & Tevatron* & DiTevatron & LHC \\
& $0.01 \mathrm{fb}^{-1}$ & $0.1 \mathrm{fb}^{-1}$ & $1 \mathrm{fb}^{-1}$ & $10 \mathrm{fb}^{-1}$ & $1 \mathrm{fb}^{-1}$ & $10 \mathrm{fb}^{-1}$ \\
& $1.8 \mathrm{TeV}$ & $1.8 \mathrm{TeV}$ & $2 \mathrm{TeV}$ & $2 \mathrm{TeV}$ & $4 \mathrm{TeV}$ & $14 \mathrm{TeV}$ \\
\hline$B_{T}(\tilde{q} \gg \tilde{g})$ & $\tilde{g}(150)$ & $\tilde{g}(210)$ & $\tilde{g}(270)$ & $\tilde{g}(340)$ & $\tilde{g}(450)$ & $\tilde{g}(1300)$ \\
$\ell^{ \pm} \ell^{ \pm}(\tilde{q} \gg \tilde{g})$ & - & $\tilde{g}(160)$ & $\tilde{g}(210)$ & $\tilde{g}(270)$ & $\tilde{g}(320)$ & $\tilde{g}(1000)$ \\
all $\rightarrow 3 \ell(\tilde{q} \gg \tilde{g})$ & - & $\tilde{g}(150-180)$ & $\tilde{g}(150-260)$ & $\tilde{g}(150-430)$ & $\tilde{g}(150-320)$ & \\
$E_{T}(\tilde{q} \sim \tilde{g})$ & $\tilde{g}(220)$ & $\tilde{g}(300)$ & $\tilde{g}(350)$ & $\tilde{g}(400)$ & $\tilde{g}(580)$ & $\tilde{g}(2000)$ \\
$\ell^{ \pm} \ell^{ \pm}(\tilde{q} \sim \tilde{g})$ & - & $\tilde{g}(180-230)$ & $\tilde{g}(325)$ & $\tilde{g}(385-405)$ & $\tilde{g}(460)$ & $\tilde{g}(1000)$ \\
all $\rightarrow 3 \ell(\tilde{q} \sim \tilde{g})$ & - & $\tilde{g}(240-290)$ & $\tilde{g}(425-440)$ & $\tilde{g}(550)$ & $\tilde{g}(550)$ & $己 \tilde{g}(1000)$ \\
$\tilde{t}_{1} \rightarrow c \tilde{\chi}_{1}^{0}$ & - & $\tilde{t}_{1}(80-100)$ & $\tilde{t}_{1}(120)$ & & & \\
$\tilde{t}_{1} \rightarrow b \tilde{\chi}_{1}^{ \pm}$ & - & $\tilde{t}_{1}(80-100)$ & $\tilde{t}_{1}(120)$ & & & \\
$\Theta\left(\tilde{t}_{1} \tilde{t}_{1}^{*}\right) \rightarrow \gamma \gamma$ & - & - & - & & & \\
$\tilde{\ell} \tilde{\ell}^{*}$ & - & $\tilde{\ell}(50)$ & $\tilde{\ell}(50)$ & $\tilde{\ell}(100)$ & & $\tilde{\ell}(250-300)$ \\
\hline
\end{tabular}

is a complex task. It requires the ability to extract signals of new physics out of data samples with potentially large Standard Model backgrounds. Realistic simulations are required that can accurately model the physics of QCD jets and the theoretical predictions of low-energy supersymmetry. The physics of low-energy supersymmetry can be treated either in the context of specific theoretical models such as those discussed in Section 3 , or in a more phenomenological approach which makes as few theoretical assumptions as is practical. Both approaches have been followed in this section. The minimal supersymmetric model (MSSM) [28] has recently been embedded in the event generator program ISAJET [29], so that all lowest order sparticle production processes and decay chains can be adequately simulated. In addition, ISAJET now allows the input of a mixed GUT and weak scale parameter set $\left(m_{0}, m_{1 / 2}, A_{0}, \tan \beta, \operatorname{sgn}(\mu), m_{t}\right)$, for which a complete superparticle spectrum is generated using renormalization group equations with radiative electroweak symmetry breaking, under the assumption of gauge coupling unification at $M_{G U T} \sim 2 \times 10^{16} \mathrm{GeV}$, and universal GUT scale soft supersymmetry breaking terms. In addition, versions of ISAJET past 7.11 include all lowest order $e^{+} e^{-} \rightarrow$ SUSY reactions, so the effect of cascade decays can be explored at future $e^{+} e^{-}$colliders [30].

Much effort has gone into examining the superparticle mass reach and discovery potential of various proposed future colliders, to aid in decision making and prioritization for future collider facilities. A summary of these results can be found in Table 4. First, we consider the case of gluino and squark production, followed by their cascade decays via three-body $\tilde{\chi}^{ \pm}$and $\tilde{\chi}^{0}$ de- cay modes. Kamon et al. [32] have examined the mass reach in $m_{\tilde{g}}$ at the Tevatron and DiTevatron colliders using the missing transverse energy $\left(Z_{T}\right)$ signature, and found gluino mass reaches of $m_{\tilde{g}} \sim 270(450) \mathrm{GeV}$ for the Tevatron Main injector (DiTevatron), assuming that $m_{\tilde{q}} \gg m_{\tilde{g}}$. In addition, ref. [33] has examined the $\tilde{g}$ mass reach via multi-lepton topologies. The LHC reach for gluinos and squarks has recently been examined in considerable detail by ATLAS studies [8]. It is generally expected that the lighter of the two top squarks $\left(\tilde{t}_{1}\right)$ is significantly lighter than the other squarks, due to the large top quark Yukawa coupling which drives diagonal stop masses to small values and enhances the off-diagonal mixing of $\tilde{t}_{L}$ and $\tilde{t}_{R}$. In this case, the present squark mass collider limits do not apply to $\tilde{t}_{1}$. In ref. [34], it is shown that Tevatron collider experiments with $100 \mathrm{pb}^{-1}$ of data can explore $m_{\tilde{t}_{1}} \sim 80-100 \mathrm{GeV}$, considerably further than the best current bounds of $\sim 45 \mathrm{GeV}$ from LEP.

Next, we examine neutralino and chargino production. A significant portion of the parameter space of minimal supergravity models can be explored at the Tevatron and DiTevatron colliders by searching for the clean trilepton plus $B_{T}$ signal from $\tilde{\chi}_{1}^{ \pm} \tilde{\chi}_{2}^{0} \rightarrow 3 \ell$ production [31]. In Table 4, we present the mass reach of the neutralino and chargino searches in terms of an equivalent gluino mass. The latter is obtained under the assumption of unified soft gaugino masses at the GUT scale (although there is some additional dependence on other supersymmetric model parameters). Note that the DiTevatron does not significantly increase the mass reach in the 36 chantnel beyond that of the Tevatron Main Injector, altholigh further optimization of cuts may be possible. Thi- is 
in contrast to the mass reach for gluinos and squarks via $E_{T}$ searches, which is significantly higher for the DiTevatron. The clean trilepton signal has also been examined in ref. [31] for the LHC. Note that it is not possible to probe $m_{\tilde{g}}>\sim 600-700 \mathrm{GeV}$ via $3 \ell+\not T_{T}$ mode at the LHC due to the turn on of the $\widetilde{\chi}_{2}^{0} \rightarrow \widetilde{\chi}_{1}^{0} h$ or $\widetilde{\chi}_{2}^{0} \rightarrow \widetilde{\chi}_{1}^{0} Z$ decay modes which spoil the signal. However, the dilepton spectrum from $\tilde{\chi}_{2}^{0} \rightarrow \widetilde{\chi}_{1}^{0} \ell \vec{\ell}$ decay can be used at the LHC to measure $m_{\widetilde{\chi}_{2}^{0}}-m_{\widetilde{\chi}_{1}^{0}}$ with significant precision. Finally, if $R$-violating $\widetilde{\chi}_{1}^{0}$ decays are present (via baryon or lepton number violating interactions), then sparticle mass reaches can change significantly to either higher or lower values [35].

The search for sleptons at hadron colliders was addressed in ref. [36]. It was shown that it would be difficult to search at the Tevatron for $m_{\tilde{e}}$ much beyond $50 \mathrm{GeV}$ (and beyond $100 \mathrm{GeV}$ at $\mathrm{TeV}^{*}$ ), due to low signal rates and irreducible backgrounds. In contrast, at the LHC stiffer cuts may be made; in this case slepton masses up to $250-300 \mathrm{GeV}$ can be detected.

Tevatron signals within the constrained supergravity framework have been discussed in ref. [37]. Because all signals are determined by just four parameters, the various SUSY processes are strongly correlated, and may provide an experimental probe of GUT scale assumptions. For example, ref. [38] argues that in the parameter regime where sleptons are much lighter than squarks, the Main Injector discovery limit may reach $m_{\tilde{g}} \sim 500 \mathrm{GeV}$ via the enhanced multi-lepton signals from supersymmetric particle production and their subsequent decay chains.

\subsection{Supersymmetry at $e^{+} e^{-}$Colliders}

At an $e^{+} e^{-}$collider, discovery of superparticles is relatively easy up to the kinematic limit. There is a vast literature concerning the discovery reach at LEP-II [39]. Recent works discuss measurement of SUSY parameters rather than discoveries alone. For instance with a chargino pair production alone, one can put stringent constraints on the parameter space $\left(M_{1}, M_{2}, \mu, \tan \beta, m_{\tilde{l}}\right)$ using the cross section, forward-backward asymmetry and branching fractions, where the sensitivity on particular parameters depends on the actual parameter values [40].

Experimentation at linear $e^{+} e^{-}$colliders would be richer due to the higher energies and beam polarization with the following characteristics: (i) small beam energy spread (better than $1 \%$ ) even after including initial state radiation and beamstrahlung effects, (ii) clean environment basically without underlying events even with photon induced hadronic processes, (iii) high beam polarization of $90 \%$ and beyond. The virtues of polarization are two-fold. First, use of polarization can suppress the background substantially by as much as two orders of magnitudes, resulting in a very pure signal sample appropriate for precision studies. Second, one basically doubles the number of experimental observables by using both polarization states, which enables the efficient measurements of various parameters.

A detector simulation of $e^{+} e^{-} \rightarrow \tilde{\mu}^{+} \tilde{\mu}^{-}$based on an ALEPH-type detector at $\sqrt{s}=500 \mathrm{GeV}$ and $\int \mathcal{L} d t=$ $20 \mathrm{fb}^{-1}$ gives a $5 \sigma$ signal for $\tilde{\mu}$ for $m_{\tilde{\mu}}$ below $230 \mathrm{GeV}$ [41]. A mass difference of $25 \mathrm{GeV}$ between slepton and the LSP is enough for detection even without employing beam polarization. The case for the chargino search is similar. It is noteworthy that both purely hadronic and mixed hadronic-leptonic modes can be used for discovery and study of charginos [42].

We show here the sample results of Monte Carlo analysis with detector simulation [43] based on JLC-I detector [44]. Fig. 1 shows the power of the polarization to obtain a pure sample ( $>99 \%$ ) of $\tilde{\mu}$ pair signals with high efficiency $(\epsilon>50 \%)$. Since the signature of a slepton pair is an acoplanar lepton pair, the main background is $W^{+} W^{-}$, which can be substantially suppressed by employing right-handed electron beam. A kinematic fit to the end points of lepton energy distribution allows us the determination of both slepton and LSP masses better than $1 \%$. The typical sensitivity of the chargino and neutralino mass measurements are shown in Fig. 2. Here the mixed hadronic-lepton mode is used, and the measurement is based on total dijet energy distribution. Using a technique to combine information from the tracking detector and calorimeters, one achieves mass measurements of the chargino and LSP at the $3 \%$ level. Similar sensitivities are expected for top and bottom squark signals [45].

The determination of superparticle masses allows us to test theoretical assumptions at various levels. For example, generation independence of slepton masses (universality) can be tested at the $1 \%$ level. More experimentally challenging is the test of the GUT-relation among gaugino masses, since the gauginos mix with higgsinos to form chargino and neutralino mass eigenstates. However, one can still test the GUT-relation at a few percent level combining slepton and chargino signals, based on the measured masses and polarization dependence of the cross sections (see Fig. 3).

\subsection{Complementarity}

Typically, $e^{+} e^{-}$colliders have lower center-of-mass energies compared to hadron machines of the same era, but their complementary character in superparticle searches is easily understood. The colored superparticles which are the main targets of the hadron colliders are supposed to be 3-4 times heavier than the colorless ones under GUT assumptions. For example, the constraints on the supersymmetric parameter space from experiments at $\operatorname{LEP}(\sqrt{s}=90 \mathrm{GeV})$ and the Tevatron $(\sqrt{s}=1.8 \mathrm{TeV})$ are comparable. This continues to be true for LEP-II and 

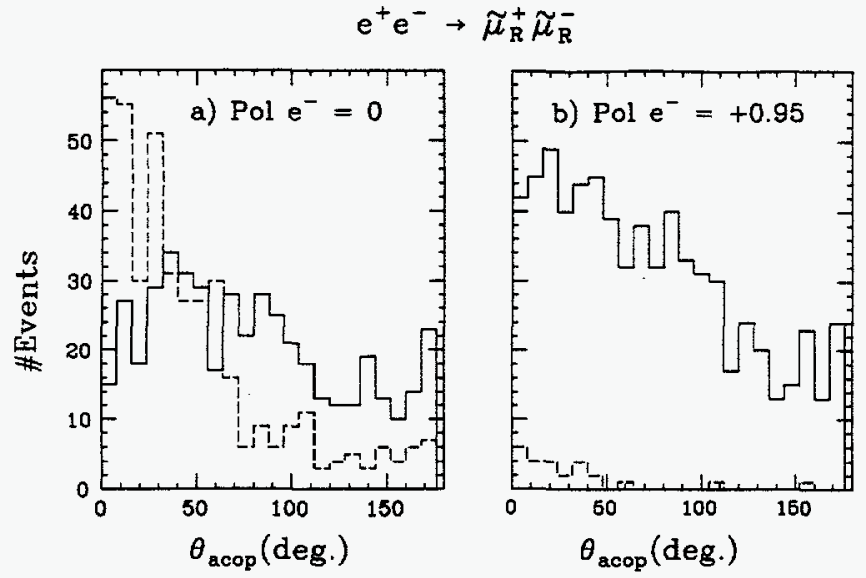

Figure 1: Acoplanarity distribution of muon pair for $E_{C M}=$ $350 \mathrm{GeV}, m_{\tilde{l}}=142 \mathrm{GeV}, \int \mathcal{L} d t=20 \mathrm{fb}^{-1}$, for the signal (solid) and standard model backgrounds (dashed). Fig. b) shows the dramatic reduction of the backgrounds using the right-handed electron beam. Taken from Ref. [43].
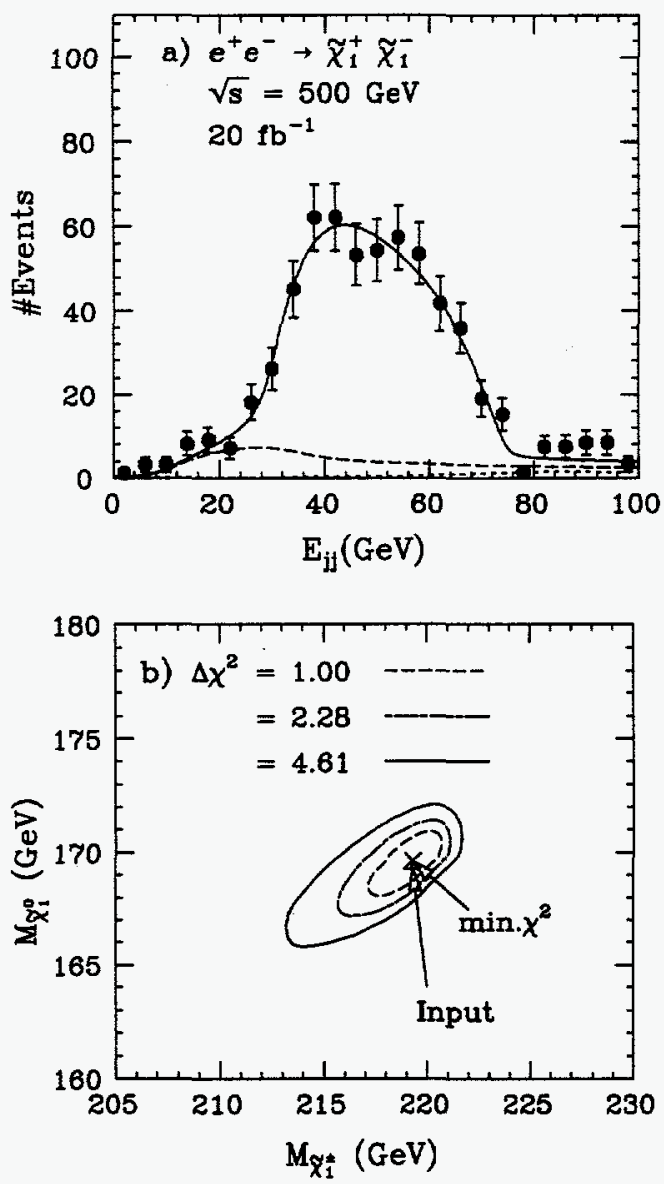

Figure 2: a) Dijet energy distribution from chargino pair production, for the Monte Carlo generated events (points), polynomial fit to the Monte Carlo (solid), contributions from the $W$-pair background (dashed) and the sum of other standard model backgrounds (dotted). b) Obtained contour on the masses of chargino and LSP. Taken from Ref. [43].

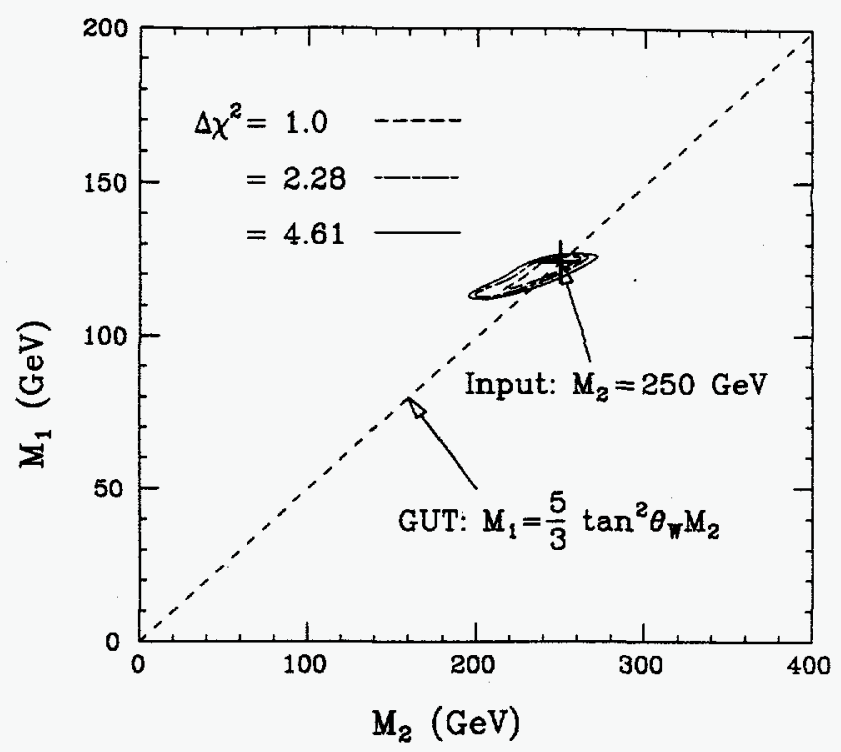

Figure 3: Test of the GUT-relation of the gaugino masses. The contours are obtained by a four-dimensional fit of $\left(M_{1}, M_{2}, \mu, \tan \beta\right)$ plane, using the LSP and chargino masses, and the production cross sections for selectrons and charginos with a right-handed electron beam. Taken from Ref. [43].

the Tevatron with Main Injector, especially in the context of models with minimal supergravity boundary condition constraints $[37,38]$. The discovery reach of an $e^{+} e^{-}$linear collider with $\sqrt{s}=1 \mathrm{TeV}$ will be again comparable to that at the LHC. But their complementary character will be more important after the discoveries of superparticles. Suppose that signals from squarks and gluinos are seen at the LHC. Then it would be a formidable task to demonstrate that they are superparticles due to the simultaneous production of many new particles and their complicated decay chains. The $e^{+} e^{-}$collider might not reach the energies where squarks could be produced; however, it would study the low-lying spectrum of superparticles (the sleptons, neutralinos and charginos) as described in the previous section. Analysis of data from the hadron collider with inputs from the $e^{+} e^{-}$collider could sort out the complex decay chains, revealing the mass spectrum and parameters of the heavier superparticles. These results would augment the measurements of the properties of light superparticles made at the $e^{+} e^{-}$collider, and would provide additional tests of GUT and/or supergravity predictions and test other model assumptions.

\section{Strongly Coupled ESB Sector: Model- Independent Approaches}

If a Higgs boson or techni-rho meson or some other direct indication of electroweak symmetry breaking is not found at some future accelerator, one would like to know 
whether it is still possible to learn something about electroweak symmetry breaking by studying $W W$ scattering. In this scenario, whatever is responsible for giving the $W$ and $Z$ bosons their masses occurs at a high energy scale and most probably gives rise to a strongly interacting sector where perturbation theory is not applicable. Fortunately, one can still analyze the low energy behavior of the theory with a generalization of the $\pi \pi$ scattering theorems derived by Weinberg cast in the form of chiral perturbation theory.

The low energy theorems can easily be generalized from pions to the longitudinal components of the $W$ and $Z$ gauge bosons. These scattering theorems are only valid at low energy, that is $\sqrt{s}<<\left(M_{\text {Res }}, 4 \pi v\right)$, where $M_{\text {Res }}$ is the mass of the lowest lying resonance interacting with the longitudinal $W$ 's and $Z$ 's. The attraction in this approach is that it is valid for any mechanism of electroweak symmetry breaking. It is also possible to couple the chiral Lagrangian describing the low energy interactions of the gauge bosons with a (color-singlet) vector meson (the BESS model-equivalence) or with a scalar particle (a Higgs scalar type of models). This description of vector mesons and scalars coupled to longitudinal gauge bosons relies only on the symmetry of the theory and so is independent of the details of specific models.

Lastly, we discuss a model with a "hidden" symmetry breaking sector, one in which the electroweak symmetry breaking sector has a large number of particles. The theory can be strongly interacting and the total $W$ and $Z$ cross sections in this model are large: most of the cross section is for the production of particles other than the $W$ or $Z$. In such a model, discovering the electroweak symmetry breaking sector depends on the observation the other particles and the ability to associate them with symmetry breaking.

\subsection{Strongly Coupled ESB Sector at the LHC}

In what follows, we assume the $\mathrm{LHC}$ runs at $14 \mathrm{TeV}$ with a luminosity of $10^{34} \mathrm{~cm}^{-2} \mathrm{sec}^{-1}$ (an annual integrated luminosity $100 \mathrm{fb}^{-1}$ ).

\section{Vector Resonance Signal}

A vector meson with a mass up to $m_{\rho}=2.5 \mathrm{TeV}$ can be found at the LHC through the $W^{ \pm} Z$ channel [46]. One would need to run the LHC for about 2 years to collect about 10 leptonic events, after kinematical cuts to effectively suppress the backgrounds.

Complementarity of Vector Resonant and Non-resonant $W W$ Scattering:

Ref. [47] considered a vector-dominance model for three sets of mass-width parameters: $\left(m_{\rho}, \Gamma_{\rho}\right)=$ $(1.78,0.33),(2.52,0.92)$, and $(4.0,0.98)$. Their criterion for a significant signal $(S)$ with a background $(B)$ is $\sigma^{\uparrow} \equiv S / \sqrt{B} \geq 5 ; \sigma^{\downarrow} \equiv S / \sqrt{S+B} \geq 3 ; S \geq B$. The complementarity here implies that one observes either a low mass $(I, J)=(1,1)$ resonance via the $W^{ \pm} Z$ channel, or significant enhancement via the $W^{ \pm} W^{ \pm}$channel with $I=2$. From Table 5, one sees that the worst case among those parameter choices is at $m_{\rho}=2.52 \mathrm{TeV}$, where an integrated luminosity of at least $105 \mathrm{fb}^{-1}$ slightly more than the LHC annual luminosity-is required for the $W^{ \pm} W^{ \pm}$channel to meet their discovery criterion. ATLAS collaboration [8] carried out a Monte Carlo study for $W^{+} W^{+}$channel including detector simulations, obtaining qualitatively similar results.

Table 5: Minimum luminosity to satisfy observability criterion for $W^{ \pm} Z$ and $W^{ \pm} W^{ \pm}$channels at the LHC. Each entry contains $\mathcal{L}_{M I N}$ in $\mathrm{fb}^{-1}$, the number of signal/background events per $100 \mathrm{fb}^{-1}$.

\begin{tabular}{|cccc|}
\hline$m_{\rho}(\mathrm{TeV})$ & 1.78 & 2.52 & 4.0 \\
\hline \hline & $44 \mathrm{fb}^{-1}$ & $325 \mathrm{fb}^{-1}$ & No \\
$W^{ \pm} Z$ & $38 / 20$ & $5.8 / 3.4$ & Signal \\
\hline & $142 \mathrm{fb}^{-1}$ & $105 \mathrm{fb}^{-1}$ & $77 \mathrm{fb}^{-1}$ \\
$W^{ \pm} W^{ \pm}$ & $12.7 / 6.0$ & $15.9 / 5.8$ & $22.4 / 8.9$ \\
\hline
\end{tabular}

\section{All-Channel Comparison for WW Scattering}

Ref. [48] compared all-channel $W W$ scattering at $\sqrt{s}=40 \mathrm{TeV}$ and $16 \mathrm{TeV}$, and new analysis at $14 \mathrm{TeV}$ is in progress. In particular, the signal for a $1 \mathrm{TeV}$ Higgs boson may be observable through the leptonic channels $H \rightarrow W W \rightarrow l \nu l \nu$ and $Z Z \rightarrow l \bar{l} \nu \bar{\nu}$, to add to the clean but low rate $Z Z \rightarrow 4 l$ mode.

ATLAS collaboration [8] also studied a $1 \mathrm{TeV}$ Higgs boson signal via the decay mode $H \rightarrow W W \rightarrow l \nu j j$ and $Z Z \rightarrow l \bar{l} j j$. It was found that after identifying one $W$ in the leptonic mode and one $W$ in the di-jet mode with $M_{j j} \sim m_{W}$, with a tagged forward jet and a veto on all other central jets, the $W W \rightarrow \ell \nu j j$ channel gives a viable signal with statistical significance above $6.8 \sigma$.

\subsection{Strongly Coupled ESB Sector at the NLC}

Our standard NLC has an energy $\sqrt{s}=1.5 \mathrm{TeV}$ and an integrated luminosity of $190 \mathrm{fb}^{-1}$ per year.

\section{Vector Resonance Signal}

At the NLC a vector resonance would be probed most effectively via $e^{+} e^{-} \rightarrow W_{L}^{+} W_{L}^{-}$. This can be generically described [49] by an Omnés function with a complex form factor $F_{T}$. An infinitely massive vector resonance is equivalent to the case where the $W_{L}^{+} W_{L}^{-}$scattering amplitude is described by the Low Energy Theorem (LET).

Fig. 4 shows the results in Ref. [50] for a technirholike vector resonance in the $e^{+} e^{-} \rightarrow W^{+} W^{-}$process. 
In one year at the NLC, technirhos of arbitrarily large mass can be distinguished from the weakly-interacting SM. An integrated luminosity of $225 \mathrm{fb}^{-1}(1.2$ years at design luminosity) would yield $7.1 \sigma, 5.3 \sigma$, and $5.0 \sigma$ signals for a $4 \mathrm{TeV}$ techni-rho, a $6 \mathrm{TeV}$ techni-rho, and LET, respectively. As was the case for the LHC of Table 5 , these results assume statistical errors only. The systematic errors for the $e^{+} e^{-} \rightarrow W^{+} W^{-}$results will be small compared to the statistical error, since the analysis consists of a background-free maximum likelihood fit of angular distributions.

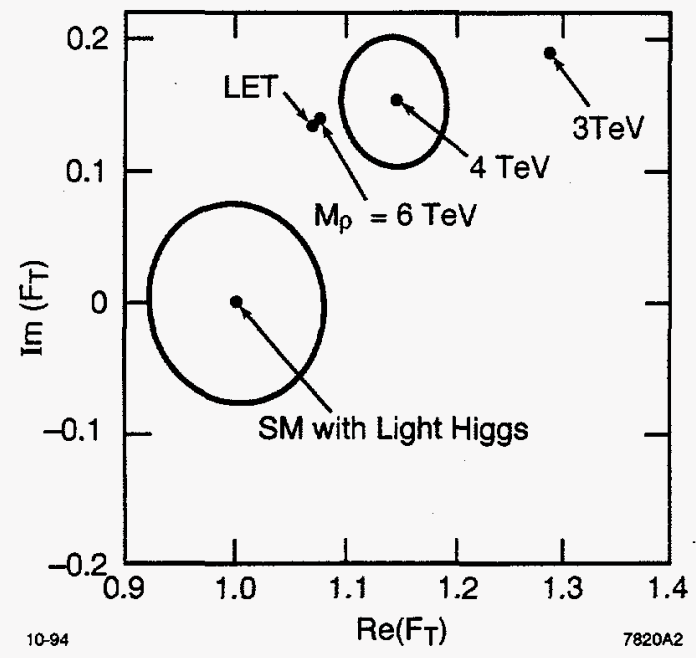

Figure 4: Confidence level contours for $\operatorname{Re}\left(F_{T}\right)$ and $\operatorname{Im}\left(F_{T}\right)$ at c.m. energy $1.5 \mathrm{TeV}$ and $\mathcal{L}=190 \mathrm{fb}^{-1}$. The contour about the light Higgs value of $F_{T}=(1,0)$ is $95 \%$ C.L. and the contour about $m_{\rho}=4 \mathrm{TeV}$ point is $68 \%$ C.L..

The processes of $e^{+} e^{-} \rightarrow W^{+} W^{-}$and $e^{+} e^{-} \rightarrow f \bar{f}^{\prime}$ via the vector resonance have also been studied in the context of the BESS model and variables such as the total cross sections, forward-backward and left-right asymmetries are used to constrain the virtual $V$ effects. For $\sqrt{s} \sim m_{\rho}$, the NLC will be very sensitive to the BESS model parameters [51].

\section{WW Fusion Processes}

$W W$ fusion processes open more channels to study the strongly coupled ESB sector beyond a vector resonance. Ref. [52] demonstrates that the signal for a 1 $\mathrm{TeV}$ Higgs boson should be observable at a $12 \sigma$ level, for $\sqrt{s}=1.5 \mathrm{TeV}$ and $200 \mathrm{fb}^{-1}$. Furthermore, the ratio $R \equiv \sigma\left(W^{+} W^{-} \rightarrow W^{+} W^{-}\right) / \sigma\left(W^{+} W^{-} \rightarrow Z Z\right)$ may provide hints that help to distinguish different strongly coupled ESB sector models, such as a scalar resonance $(R>1)$, a vector resonance $(R \gg 1)$, or a Low Energy Theorem amplitude $(R<1)$.

Due to the relatively clean environment in $e^{+} e^{-}$col- lisions, cross sections for background processes may be measured rather well for both the normalization as well as the shape of the distribution, so that statistically significant deviation from the SM background may be easily identified as new physics signal. One example is the "Hidden Sector" [53], which may be impossible to observe in hadronic collision environment but possibly feasible in $e^{+} e^{-}$collisions.

\section{Searches at $\gamma \gamma$ Colliders}

The heavy Higgs boson and other strong scattering signals in longitudinal gauge boson channels can be investigated at the NLC at $\sqrt{s}=1.5 \mathrm{TeV}$ operating in the $\gamma \gamma$ collider mode [54]. The processes considered here involve two gauge boson final states $\gamma \gamma \rightarrow Z Z$, $\gamma \gamma \rightarrow W^{+} W$, and four gauge boson final states $\gamma \gamma \rightarrow$ $W^{+} W^{-} W^{+} W^{-}, W^{+} W^{-} Z Z$ [55]. The four gauge boson final state processes seem to be more promising in searching for strongly coupled ESB dynamics as compared with the two gauge boson final states, although all the above processes are plagued by large backgrounds consisting of transversely produced gauge bosons [54]. This issue has been addressed recently in Refs. [56,57]. For example, Ref. [56] finds that with luminosity of $200 \mathrm{fb}^{-1}$, a 1.5 $\mathrm{TeV} e^{+} e^{-}$collider operating as a $\gamma \gamma$ collider can successfully observe a heavy Higgs boson with mass up to $700 \mathrm{GeV}$. To obtain a reach in the Higgs boson mass up to $1 \mathrm{TeV}$, a $2 \mathrm{TeV} e^{+} e^{-}$collider is required with luminosity of $200 \mathrm{fb}^{-1}$. The results were obtained taking into account the back-scattered photon luminosity spectrum. It is also found that a $2 \mathrm{TeV}$ linear collider in the $\gamma \gamma$ mode is roughly equivalent to a $1.5 \mathrm{TeV} e^{+} e^{-}$collider in searching for heavy Higgs boson physics (roughly scales as $\sqrt{s_{\gamma \gamma}} \sim 0.8 \sqrt{s_{e e}}$ ). The viability of this signal still requires that the decays of the $W$ and $Z$ bosons be incorporated, and detector simulations be performed to determine a realistic acceptance.

\section{Strongly Coupled ESB Sector: Implications of Models}

\subsection{Ingredients of Techni-models}

In theories of dynamical electroweak symmetry breaking (ESB), such as technicolor [58], ESB is due to chiral symmetry breaking in an asymptotically-free, stronglyinteracting, gauge theory with massless fermions. These theories attempt to understand electroweak symmetry breaking without introducing any scalar particles such as a Higgs boson. Instead, the symmetry breaking is modeled after that which occurs in QCD when the $S U(3)_{c}$ color gauge group becomes strong. In QCD, the breaking of the chiral symmetry gives rise to pions. In the simplest technicolor theory one introduces a left-handed weak-doublet of "technifermions", and the corresponding 
right-handed weak-singlets, which transform as the fundamental $[N]$ representation of a strong $S U(N)_{T C}$ technicolor gauge group. The global chiral symmetry of the technicolor interactions is then $S U(2)_{L} \times S U(2)_{R}$. When the technicolor interactions become strong, the chiral symmetry is broken to the diagonal subgroup, $S U(2)_{V}$, producing three Nambu-Goldstone bosons, which then yield the correct $W$ and $Z$ masses.

However, the symmetry breaking sector must also couple to the ordinary fermions, allowing them to acquire mass. In models of a strong ESB sector there must either be additional flavor-dependent gauge interactions, the so-called "extended" technicolor (ETC) interactions, or Yukawa couplings to scalars which communicate the breaking of the chiral symmetry of the technifermions to the ordinary fermions. The most popular type of strong ESB model that attempts to explain the masses of all observed fermions contains an entire family of technifermions with Standard Model gauge couplings.

Since these models contain more than one doublet of technifermions, they have a global symmetry group larger than $S U(2)_{L} \otimes S U(2)_{R}$. Therefore chiral symmetry breaking produces additional (pseudo-) NambuGoldstone bosons (PNGBs). Furthermore, the models typically possess a larger variety of resonances than the one-doublet model. There will be heavy ( 1-2 TeV) vector meson resonance with the quantum numbers of the $\rho, \omega$ etc. The phenomenology of color-neutral resonances with the quantum numbers of the $\rho$ is discussed in Section 5. In the case of color-neutral PNGBs the phenomenology is much like that of the extra scalars in "twoHiggs" doublet models. Therefore we will focus on the colored resonances and PNGBs occuring in most technicolor models. These models have a possibly unique signature: resonances associated with the electroweak symmetry breaking sector which are strongly produced. Hadron colliders will be especially important for searching for signatures of such colored particles associated with ESB.

\subsection{Theoretical Challenges for Technicolor Model Building}

Models incorporating ETC interactions ran into trouble with flavor changing neutral currents (FCNC's) early on. Assuming technicolor behaved like a scaled-up version of QCD it was found that it is impossible to generate a large enough mass for the $s$ quark (much less the $c$ quark) while avoiding FCNC's. One possible solution is to make the TC gauge coupling run slower than in QCD (i.e. make it 'walk'). This makes the technifermion condensate much larger than scaling from QCD suggests, so that for a fixed quark or lepton mass, the necessary mass scale for ETC gauge bosons is increased, suppressing FCNC's. A second solution is to build a Glashow-Illiopoulos-Maiani
(GIM) symmetry into the ETC theory (sometimes called TechniGIM).

A further problem for ETC models is producing a large isospin splitting for the $t$ and $b$ quark masses without causing large isospin splitting in the $W$ and $Z$ masses. Isospin splitting in the gauge sector, as described by the radiative correction parameter $T$ is tightly constrained by experiment. ${ }^{a}$

A current challenge for TC models is that models with QCD-like dynamics tend to produce large positive contributions to the electroweak radiative correction parameter $S$, while experiments tend to prefer small $S$. Since the discrepancy grows with the number of technicolors and the number of technidoublets, one way to evade the problem is to limit the number of technifermions contributing to ESB. Alternatively one can invoke mechanisms yielding a negative contribution to $S$ : the technifermions may have exotic electroweak quantum numbers or the TC dynamics can be sufficiently unlike QCD so as to invalidate the naive scaling-up of QCD phenomenology. Such alternatives usually also rely on some form of isospin breaking. In multiscale models isospin breaking at the lowest ESB scale can produce a negative contribution to $S$ without infecting the $T$ parameter, which is sensitive to isospin breaking at the highest ESB scale.

The most recent problem for ETC models arises from the measurement of the $Z \rightarrow b \bar{b}$ partial width at LEP. The experiments find a partial width which is slightly larger than the Standard Model prediction, while almost all the ETC models that have been constructed so far produce a correction which further reduces this width. The sign of the correction is a consequence of the almost universal assumption that $S U(2)_{L}$ commutes with the ETC gauge group. The alternative, where $S U(2)_{L}$ is embedded in the ETC gauge group, may be interesting, since this reverses the sign of the correction.

\subsection{Phenomenology of Pseudo-Goldstone Bosons}

Many ETC models are expected to include a spectrum of pseudo-Nambu-Goldstone bosons ( $P$ 's) and vector resonances $\left(\rho_{T}\right.$ 's and $\left.\omega_{T}\right)$ that may carry color quantum numbers. These particles may be classified by their $S U(3)_{C}$ and $S U(2)_{V}$ quantum numbers as indicated (for the $P$ 's) in the first column of Table 6 , using the nomenclature of ref. [59]. Since these particles are strongly produced, they have large cross sections at the Tevatron and LHC. The color triplet $\left(P_{8}\right)$ and color octet $\left(P_{3}\right)$ pseudo-NambuGoldstone bosons will be pair produced from gluon-gluon initial states in hadron colliders and will give rise to 4 jet and $t \bar{t}$ final states, since in many models the PNGBs couple preferentially to heavy fermions. Since $P_{3}$ and $P_{8}$

\footnotetext{
${ }^{a}$ The radiative correction parameters, $S, T$ and $U$ are defined in the chapter on Precision Tests of Electroweak Physics.
} 
Table 6: Discovery reach (in $\mathrm{GeV}$ ) of different accelerators for particles associated with realistic models of a strong ESB sector.

\begin{tabular}{|lc|c|c|c|c|c|}
\hline & $\left(S U(3)_{C}\right.$, & & & & & \\
Particle & $\left.S U(2)_{V}\right)$ & Tevatron & LHC & LEP I & LEP II & NLC-1000 \\
\hline$P^{0 \prime}$ & $(1,1)$ & - & $110-150^{a}$ & $8^{b} ; 28^{b}$ & $--^{c}$ & $-{ }^{c, d}$ \\
$P^{0}$ & $(1,3)$ & - & - & - & - & $-^{d}$ \\
$P^{+} P^{-}$ & $(1,3)$ & - & $400^{e}$ & $45^{f}$ & $100^{g}$ & $500^{g}$ \\
\hline$P_{8}^{0 \prime}\left(\eta_{T}\right)$ & $(8,1)$ & $400-500^{h}$ & $325^{i}$ & - & - & - \\
$P_{8}^{0} \mathrm{~d}$ & $(8,3)$ & $10-20^{i}$ & $325^{i, j}$ & - & - & - \\
$P_{8}^{+} P_{8}^{-}$ & $(8,3)$ & $10-20^{i, j}$ & $325^{i, j}$ & $45^{f}$ & $100^{g}$ & $500^{g}$ \\
\hline$P_{3}^{+} P_{3}^{-}$ & $(3,3)$ & $-j$ & $-j$ & & $100 \mathrm{GeV}^{g}$ & $500 \mathrm{GeV}^{g}$ \\
\hline
\end{tabular}

\footnotetext{
${ }^{a}$ Decay mode $P^{0 \prime} \rightarrow \gamma \gamma$, similar to a light neutral Higgs [60].

${ }^{b}$ Decay mode $Z \rightarrow \gamma P^{0}$, assuming an one-family model, with $N_{T C}=7$ and $N_{T C}=8$ respectively; no reach for $N_{T C}<7$; for larger $Z \gamma P^{0 \prime}$ couplings, the discovery reach extends to $65 \mathrm{GeV}$ [61].

c No reach for one-family model; possibility of reach for the Lane-Ramana [62] multiscale model in the processes $e^{+} e^{-} \rightarrow \mathrm{Pr}_{\gamma}, \mathrm{Pe}^{+} e^{-}[63]$.

$d$ The analogous discovery reach for a CP-odd Higgs scalar can be greatly improved using the NLC operating in the $\gamma \gamma$ collider mode (with polarized photon beams) [64].

Estimated from work on charged Higgs $\left(g b \rightarrow t H^{-} \rightarrow t \vec{t} b\right)$ for $\tan \beta \simeq 1$ [65].

${ }^{f}$ LEP I kinematical limit.

$g$ Kinematical limits for LEP200 and the NLC-1000.

${ }^{h}$ Contribution to the $\bar{t} t$ cross section in multiscale models [66].

QCD pair production of colored PNGBs with decay into 4 jets [67].

$j$ QCD pair production of colored PNGBs with decay into $t \bar{t}, t \bar{b}, \tau^{+} \tau^{-}$or $\tau \nu_{\tau}$ may allow higher reach in mass. This has yet to be studied.
}

couple to the $Z$ boson they can also be pair produced in $e^{+} e^{-}$colliders, which are expected to be sensitive to PNGB masses up to the kinematic limit. The LHC will be sensitive to $P_{8}$ masses up to $\sim 325 \mathrm{GeV}$, while an NLC-1000 would reach $\sim 500 \mathrm{GeV}$. Since colored PNGBs receive their masses from QCD interactions and typically have masses in the 100-300 GeV range, both the LHC and NLC-1000 can probe the interesting region. The expected discovery potential of various colliders for the pseudo-Nambu-Goldstone bosons is shown in Table 6.

While as yet no completely realistic technicolor model exists, the models all share the general feature of having a rich spectrum of scalar and vector particles which can be probed at either the LHC or a high energy $e^{+} e^{-}$collider.

\section{New Gauge Bosons}

\subsection{Introduction}

Extended gauge symmetries and the associated heavy neutral $\left(Z^{\prime}\right)$ and/or charged $\left(W^{\prime}\right)$ gauge bosons are a feature of many extensions of the Standard Model (SM). We survey and compare the discovery potential of the experiments that will be performed over the next decade
(Tevatron and LEP-II) and future facilities that are being planned and considered for the period beyond.

We present results for heavy gauge boson physics for the following representative models: $Z_{\chi}$ in $S O_{10} \rightarrow$ $S U_{5} \times U_{1 \chi}, Z_{\psi}$ in $E_{6} \rightarrow S O_{10} \times U_{1 \psi}, Z_{\eta}=\sqrt{3 / 8} Z_{\chi}-$ $\sqrt{5 / 8} Z_{\psi}$ in superstring inspired models in which $E_{6}$ breaks directly to a rank 5 group [68], as well as $Z_{L R}$ and $W_{L R}^{\prime}$ in the LR symmetric models [69]. For simplicity, we assume that the neutrino produced in the $W^{\prime}$ decay does not produce visible energy in the detector.

\subsection{Discovery Limits of Extra Gauge Bosons}

Current limits on the mass of new heavy gauge bosons are relatively weak. In Table 7 the direct bounds on $Z^{\prime}$ production from the main production channel $p \bar{p} \rightarrow$ $Z^{\prime} \rightarrow e^{+} e^{-}$from current Tevatron data as well as the indirect unconstrained (no assumption on the Higgs sector) and constrained (specific assumption on the Higgs sector) bounds from a global analysis of electro-weak data are presented [70]. From the non-observation of $p p \rightarrow W^{\prime} \rightarrow e \nu_{e}$ at the Tevatron, the CDF Collaboration concludes that $M_{W_{L_{R}}^{\prime}}>652 \mathrm{GeV}$ [71]. In contrast, the indirect constrained [unconstrained] Tevatron bounds on $M_{W_{L R}^{\prime}}$ are in the $1.4 \mathrm{TeV}[300 \mathrm{GeV}]$ region.

Discovery limits for new gauge bosons at future col- 
Table 7: Current constraints on $M_{Z^{\prime}}$ (in $\mathrm{GeV}$ ) for typical models (see ref. [70] for details) from direct production at the Tevatron $\left(\mathcal{L}_{\text {int }}=19.6\right.$ $\left.\mathrm{pb}^{-1}\right)$, as well as indirect limits (95\% C.L.) from a global electro-weak analysis.

\begin{tabular}{|r|ccc|}
\hline Models & Direct & $\begin{array}{c}\text { Indirect } \\
\text { (unconstrained) }\end{array}$ & $\begin{array}{c}\text { Indirect } \\
\text { (constrained) }\end{array}$ \\
\hline$\chi$ & 425 & 330 & 920 \\
$\psi$ & 415 & 170 & 170 \\
$\eta$ & 440 & 210 & 600 \\
$L R$ & 445 & 390 & 1380 \\
\hline
\end{tabular}

Table 8: Discovery limits for $M_{Z^{\prime}}$ (in $\mathrm{GeV}$ ) for typical models achievable at proposed hadron and $e^{+} e^{-}$colliders. At hadron colliders the discovery limits for $Z^{\prime}\left[W^{\prime+}+W^{\prime-}\right]$ are for typical models with 10 events in $e^{+} e^{-}+\mu^{+} \mu^{-}\left[\left(e^{+} \nu_{e}+e^{-} \bar{\nu}_{e}\right)+\left(\mu^{+} \nu_{\mu}+\mu^{-} \bar{\nu}_{\mu}\right)\right]$ from Drell-Yan production of the gauge boson. For $e^{+} e^{-}$colliders the discovery limits are the $99 \%$ C.L. from a $\chi^{2}$ fit of the observables: $\sigma\left(e^{+} e^{-} \rightarrow \mu^{+} \mu^{-}\right), R^{h a d}=\sigma^{h a d} / \sigma^{\ell}, A_{F B}^{\ell}$, and $A_{L R}^{\ell}$.

\begin{tabular}{|l|cc|rrrrr|}
\hline Collider & $\sqrt{s}[\mathrm{TeV}]$ & $\mathcal{L}_{\text {int }}\left[\mathrm{fb}^{-1}\right]$ & $\chi$ & $\psi$ & $\eta$ & $L R$ & $W_{L R}^{\prime}$ \\
\hline Tevatron $(p \bar{p})$ & 1.8 & 1 & 775 & 775 & 795 & 825 & 920 \\
Tevatron ${ }^{*}(p \bar{p})$ & 2 & 10 & 1040 & 1050 & 1070 & 1100 & 1180 \\
Di-Tevatron $(p \bar{p})$ & 4 & 20 & 1930 & 1940 & 1990 & 2040 & 2225 \\
LHC $(p p)$ & 14 & 100 & 4380 & 4190 & 4290 & 4530 & 5310 \\
\hline LEP-II $\left(e^{+} e^{-}\right)$ & 0.2 & 0.5 & 695 & 269 & 431 & 493 & \\
NLC $\left(e^{+} e^{-}\right)$ & 0.5 & 50 & 3340 & 978 & 1990 & 2560 & \\
NLC $\left(e^{+} e^{-}\right)$ & 1 & 200 & 6670 & 1940 & 3980 & 5090 & \\
NLC $\left(e^{+} e^{-}\right)$ & 2 & 200 & 9560 & 3150 & 5830 & 7210 & \\
\hline
\end{tabular}

Table 9: Values of the "normalized" couplings (1) for the typical models. The statistical error bars indicate how well the coupling could be measured at the $\operatorname{LHC}\left(\sqrt{s}=14 \mathrm{TeV}, \mathcal{L}_{\text {int }}=100 \mathrm{fb}^{-1}\right)$ for $M_{Z^{\prime}}=1 \mathrm{TeV}$.

\begin{tabular}{|c|cccc|}
\hline & $\chi$ & $\psi$ & $\eta$ & $L R$ \\
\hline$\gamma_{L}^{\ell}$ & $0.9 \pm 0.016$ & $0.5 \pm 0.02$ & $0.2 \pm 0.012$ & $0.36 \pm 0.007$ \\
$\gamma_{L}^{q}$ & 0.1 & 0.5 & 0.8 & 0.04 \\
$\tilde{U}$ & $1 \pm 0.16$ & $1 \pm 0.14$ & $1 \pm 0.08$ & $37 \pm 6.6$ \\
$\tilde{D}$ & $9 \pm 0.57$ & $1 \pm 0.22$ & $0.25 \pm 0.16$ & $65 \pm 11$ \\
\hline
\end{tabular}

Table 10: The value of the couplings (2) for typical models and statistical error bars as determined from probes at the NLC $\left(\sqrt{s}=500 \mathrm{GeV}, \mathcal{L}_{i n t}=20 \mathrm{fb}^{-1}\right) . M_{Z^{\prime}}=1 \mathrm{TeV} .100 \%$ heavy flavor tagging efficiency and $100 \%$ longitudinal polarization of the electron beam is assumed for the first set of error bars, while the error bars in parentheses are for the probes without polarization.

\begin{tabular}{|c|cccc|}
\hline & $\chi$ & $\psi$ & $\eta$ & $L R$ \\
\hline$P_{V}^{\ell}$ & $2.0 \pm 0.08(0.26)$ & $0.0 \pm 0.04(1.5)$ & $-3.0 \pm 0.5(1.1)$ & $-0.15 \pm 0.018(0.072)$ \\
$P_{L}^{q}$ & $-0.5 \pm 0.04(0.10)$ & $0.5 \pm 0.10(0.2)$ & $2.0 \pm 0.3(1.1)$ & $-0.14 \pm 0.037(0.07)$ \\
$P_{R}^{u}$ & $-1.0 \pm 0.15(0.19)$ & $-1.0 \pm 0.11(1.2)$ & $-1.0 \pm 0.15(0.24)$ & $-6.0 \pm 1.4(3.3)$ \\
$P_{R}^{d}$ & $3.0 \pm 0.24(0.51)$ & $-1.0 \pm 0.21(2.8)$ & $0.5 \pm 0.09(0.48)$ & $8.0 \pm 1.9(4.1)$ \\
$\epsilon_{A}$ & $0.071 \pm 0.005(0.018)$ & $0.121 \pm 0.017(0.02)$ & $0.012 \pm 0.003(0.009)$ & $0.255 \pm 0.016(0.018)$ \\
\hline
\end{tabular}


liders are shown in Table 8 [72]. The LHC and a high luminosity $1 \mathrm{TeV} e^{+} e^{-}$collider have discovery limits for a $Z^{\prime}$ which are in many ways comparable. Both measurements have strengths and weaknesses. The limits obtained from the LHC are robust, in the sense that they are obtained from a direct measurement with little background, but they are, however, dependent on the total width of the $Z^{\prime}$ which is sensitive to assumptions on the particle content of the model. In contrast, limits obtained for the NLC are indirect, based on statistical deviations from the Standard Model and are therefore both more model dependent and dependent on having the systematic errors under control, but do not depend on the unknown particle content of the model.

If a $Z^{\prime}$ were discovered, its mass could be measured without any ambiguity at a hadron collider from the invariant mass of the lepton pairs which signalled the $Z^{\prime}$ discovery. In contrast, at the NLC it is the ratio $\left(g / M_{Z}^{\prime}\right)^{2}$ which enters, so that extracting the $Z^{\prime}$ mass is very model dependent. If evidence were found for a $Z^{\prime}$ at the NLC, a model independent determination of $M_{Z}$, would be imprecise at best, except for perhaps a $Z^{\prime}$ not much greater than the collider energy.

\subsection{Z' Diagnostics at the $L H C$ and NLC}

An immediate need after a $Z^{\prime}$ discovery would be to determine its origins by measuring its couplings to quarks and leptons [73]. Assuming family universality and the $Z^{\prime}$ charge commuting with the $S U(2)_{L}$ generators, the relevant quantities to distinguish between different models are the fermion charges, $\hat{g}_{L 2(R 2)}^{f}$ and the gauge coupling strength, $g_{2}$. Because the signs of the charges will be hard to determine at hadron colliders the following four "normalized" observables are used:

$$
\begin{aligned}
\gamma_{L}^{\ell} & =\frac{\left(\hat{g}_{L 2}^{\ell}\right)^{2}}{\left(\hat{g}_{L 2}^{\ell}\right)^{2}+\left(\hat{g}_{R 2}^{\ell}\right)^{2}}, \\
\gamma_{L}^{q} & =\frac{\left(\hat{g}_{L 2}^{q}\right)^{2}}{\left(\hat{g}_{L 2}^{\ell}\right)^{2}+\left(\hat{g}_{R 2}^{\ell}\right)^{2}}, \\
\tilde{U} & =\frac{\left(\hat{g}_{R 2}^{u}\right)^{2}}{\left(\hat{g}_{L 2}^{q}\right)^{2}}, \\
\tilde{D} & =\frac{\left(\hat{g}_{R 2}^{d}\right)^{2}}{\left(\hat{g}_{L 2}^{q}\right)^{2}} .
\end{aligned}
$$

The values of these couplings at the LHC for the typical models and the corresponding statistical uncertainties are given in Table 9 for $M_{Z^{\prime}}=1 \mathrm{TeV}$.

For $M_{Z^{\prime}} \simeq 2 \mathrm{TeV}$ a reasonable discrimination between models and determination of the couplings may still be possible, primarily from the forward-backward asymmetry and the rapidity ratio. However, for $M_{Z^{\prime}} \simeq 3$ $\mathrm{TeV}$ there is little ability to discriminate between models. The LHC can also address $W^{\prime}$ diagnostics for $M_{W^{\prime}} \lesssim 1-2$ $\mathrm{TeV}$.
If a $Z^{\prime}$ were produced on shell at the NLC it would be relatively straightforward to determine its properties. On the other hand, if it is far off-shell (a more likely possibility) its properties could be deduced through interference effects of the $Z^{\prime}$ propagator with the $\gamma$ and $Z$ propagators. At the NLC $\sigma^{\ell}, R^{\text {had }}=\sigma^{\text {had }} / \sigma^{\ell}$ and $A_{F B}^{\ell}$ will be measured and assuming longitudinal polarization of the electron beam is available $A_{L R}^{\ell, h a d}, A_{F B}^{\ell}$ (pol) will be possible. Although the above quantities can distinguish between different models they do not yield information on all the $Z^{\prime}$ couplings.

The following four "normalized" charges and an overall gauge coupling strength divided by the "reduced" $Z$ ' propagator are probed at the NLC:

$$
\begin{aligned}
P_{V}^{\ell} & =\frac{\hat{g}_{L 2}^{\ell}+\hat{g}_{R 2}^{\ell}}{\hat{g}_{L 2}^{\ell}-\hat{g}_{R 2}^{\ell}}, \\
P_{L}^{q} & =\frac{\hat{g}_{L 2}^{q}}{\hat{g}_{L 2}^{\ell}-\hat{g}_{R 2}^{\ell}}, \\
P_{R}^{u, d} & =\frac{\hat{g}_{R 2}^{u, d}}{\hat{g}_{L 2}^{q}}, \\
\epsilon_{A} & =\left(\hat{g}_{L 2}^{\ell}-\hat{g}_{R 2}^{\ell}\right)^{2} \frac{g_{2}^{2}}{4 \pi \alpha} \frac{s}{M_{Z^{\prime}}^{2}-s} .
\end{aligned}
$$

Recall that couplings (7.1) probed by the LHC, do not determine couplings (7.2) unambiguously. The couplings with statistical uncertainties are given in Table 10 . The $Z^{\prime}$ charges can be determined to around 10-20\%. Relative error bars are about a factor of 2 larger than the corresponding ones at the LHC.

\subsection{Complementarity}

Among existing facilities currently in operation, the Tevatron offers the highest discovery reach for new gauge bosons with masses up to the $700-900 \mathrm{GeV}$ range although LEP-II can achieve comparable limits for some models. In the longer term, hadron colliders, i.e., different upgraded versions of the Tevatron and the $\mathrm{LHC}$, as well as a high luminosity $e^{+} e^{-}$collider, i.e., the NLC, would significantly improve limits on the heavy gauge boson masses. For the typical models such limits are in the 1-2 TeV region for the Tevatron upgrades, in the 4$5 \mathrm{TeV}$ region for the $\mathrm{LHC}$, and roughly 2-10 times the center-of-mass energy for the NLC with $50 \mathrm{fb}^{-1}$.

The LHC and a high luminosity $1 \mathrm{TeV} e^{+} e^{-}$collider have discovery limits for $Z^{\prime}$ which are in many cases comparable. However, limits obtained for the LHC and the subsequent measurement of its mass are robust, in the sense that they are obtained from a direct measurement with little background. In contrast, discovery limits and mass measurements at the NLC are indirect and therefore model specific.

Once discovered, the LHC and the NLC possess complementary diagnostic power for the model independent 
determination of the $Z^{\prime}$ couplings to quarks and leptons. In conjunction, the two machines may allow for determination of the $M_{Z^{\prime}}$, an overall $Z^{\prime}$ gauge coupling strength as well as a unique determination of all the quark and lepton charges with error bars in the $10-20 \%$ range, provided $M_{Z^{\prime}} \lesssim 1-2 \mathrm{TeV}$.

\section{New particles and Interactions}

Many theories beyond the Standard Model (SM) of the electroweak and strong interactions, such as Grand Unified Theories (GUT) or composite models, require the existence of new matter particles with the possibility of new interactions not contained in the SM. These particles can be cast into three categories: new elementary fermions $[68,74,75,76,77]$, difermions $[68,78,79]$ and excited fermions $[80,81,82]$. If any signals are seen, it will be crucial to distinguish among the variety of possible new states described in this section. In general, total cross-sections, angular distributions and the polarization of final state particles (resulting from new particle decays) can be used to discriminate among the different possibilities [83].

\subsection{New Elementary Fermions}

The classic examples of new fermions include: sequential fourth generation fermions (with a right-handed neutrino state to allow for neutrino masses larger than $m_{Z} / 2$ ); vector-like fermions with both left- and right-handed components in weak isodoublets [68], mirror fermions which have the opposite chiral properties as the SM fermions [75] and isosinglet fermions such as the $\mathrm{SO}(10)$ Majorana neutrino [76].

Fermions that have the usual lepton/baryon quantum numbers but possess non-canonical $\mathrm{SU}(2)_{L} \times \mathrm{U}(1)_{Y}$ quantum numbers are called exotic fermions. They occur naturally in GUT models that contain a single representation into which a complete generation of SM quarks and leptons can be embedded. For instance, in the group $E_{6}$ each fermion generation lies in the $\mathbf{2 7}$ representation, which contains 12 new fermions in addition to the 15 chiral fermions of the SM [68].

It is conceivable that these new fermions acquire masses not much larger than the Fermi scale, if these masses are protected by some symmetry. In fact, this is necessary if the associated new gauge bosons (which are present in the same GUT model that contains the new fermions) are relatively light [84]. In the case of sequential and mirror fermions (at least in the simplest models where the ESB pattern is the same as in the SM), unitarity arguments suggest that their masses should not exceed a few hundred $\mathrm{GeV}$ [85]. These particles, if they exist, should therefore be accessible at the next generation of colliders.
Except for singlet neutrinos, the new fermions couple to the photon and/or to the weak gauge bosons $W / Z$ (and for heavy quarks, to gluons) with full strength; these couplings allow for pair production with practically unambiguous cross sections. In general, new fermions can mix with SM fermions which have the same conserved quantum numbers (such as color and electric charge). This mixing gives rise to new currents, which determine the decay properties of the heavy fermions and allow for their single production. However, flavor changing neutral currents (FCNC's) will be generated at levels inconsistent with present experiments unless intergenerational mixing is highly suppressed or absent. In the latter case, the mixing pattern simplifies considerably [86]. The remaining angles are restricted by LEP and low energy experiment data to be smaller than $\mathcal{O}(0.04-0.1)$ [86]. Note that LEP 1 sets bounds of $\sim m_{Z} / 2$ on the masses of these particles [87] (stronger mass bounds from Tevatron can be set for new quarks); masses up to $m_{Z}$ might be probed at LEP2.

The new fermions decay through mixing into massive gauge bosons plus their ordinary light partners, $F \rightarrow$ $f Z / f^{\prime} W$. For masses larger than $m_{w}\left(m_{Z}\right)$ the vector bosons will be on-shell. For small mixing angles, $\theta<$ 0.1 , the decay widths are less than $10 \mathrm{MeV}(\mathrm{GeV})$ for $m_{F}=0.1(1) \mathrm{TeV}$. The charged current decay mode is always dominant and for $m_{F} \gg m_{Z}$, it has a branching fraction of $2 / 3$.

New fermions can be pair-produced in $e^{+} e^{-}$collisions, $e^{+} e^{-} \rightarrow F \vec{F}$, through $s$-channel gauge boson exchange. The cross sections are of the order of the pointlike QED cross section and therefore, are rather large [83]. Because of their clear signatures, the detection of these particles is straightforward in the clean environment of $e^{+} e^{-}$colliders, and masses very close to the kinematical limit can be probed. Charged fermions can also be pair-produced at $\gamma \gamma$ colliders via $\gamma \gamma \rightarrow F \vec{F}$. The cross-sections are comparable (and may be larger for smaller $F$ masses) to the corresponding rates in $e^{+} e^{-}$collisions. Heavy quarks can be best searched for at hadron colliders where the production processes, $g g / q \bar{q} \rightarrow Q \bar{Q}$. give very large cross sections: at the LHC with $\sqrt{s}=14$ $\mathrm{TeV}$ and a luminosity of $10 \mathrm{fb}^{-1}$ quark masses up to 1 $\mathrm{TeV}$ can be reached [88].

If the mixing angles between the SM and heavy fermions are not too small, then singly-produced new fermions in association with their light partners may produce an observable signal. The rate for single production is very model dependent (in contrast to pair-production rates mentioned above); however, it has the potential for significantly increasing the new fermion mass reach of a given collider. In $e^{+} e^{-}$collisions, single production proceeds only via $s$-channel $Z$ exchange in the cast of quarks and second/third generation leptons, leading !n small rates. For the first generation leptons, how...... 
one has additional $t$-channel exchanges which increase the cross sections by several orders of magnitude [83]. A full simulation of the signals and the backgrounds has been recently carried out; see ref. [77].

Heavy leptons of the first generation can also be singly produced in $e p$ collisions through $t$-channel exchange. At LEP $\times \mathrm{LHC}$ with $\sqrt{s}=1.2 \mathrm{TeV}$ and $\int \mathcal{L}=2$ $\mathrm{fb}^{-1}$, the $N$ and $E$ mass reach has been considered in ref. [89]. In Left-Right models, an additional production mechanism for single $N$ production via $t$-channel $W_{R}$-exchange must be included. A detailed Monte Carlo analysis of this process has been carried out in ref. [90]. In $e^{+} e^{-}$collisions these neutrinos, can be pair produced with observable cross-sections if the $W_{R}$ bosons are not too heavy $\left(M_{W_{R}}<2 \mathrm{TeV}\right.$ at $\left.\sqrt{s}=0.5 \mathrm{TeV}\right)$. One can also search for heavy Majorana neutrinos, through their virtual effects in the process $e^{-} e^{-} \rightarrow W_{R}^{-} W_{R}^{-}$[91].

\subsection{Difermions}

Difermions are new bosons (either spin 0 or spin 1) that have unusual baryon and/or lepton quantum numbers. Examples of these particles are leptoquarks (LQ) with $\mathrm{B}= \pm 1 / 3$ and $\mathrm{L}= \pm 1$, diquarks with $\mathrm{B}= \pm 2 / 3$ and $\mathrm{L}=0$ and dileptons with $\mathrm{B}=0$ and $\mathrm{L}= \pm 2$. They occur in some GUT models (e.g., in $E_{6}$, the color triplet weak isosinglet new particle can be either a LQ or diquark) and in composite models.

In addition to the usual couplings to gauge bosons, difermions have couplings to fermion pairs which determine their decays. These couplings are a priori unknown, although one typically assumes that the couplings between different generations are negligible in order to avoid generating tree-level FCNC processes. As an example, consider the case of LQ's. A systematic description of their quantum numbers and interactions can be made by starting from an effective lagrangian with general $\mathrm{SU}(3) \times \mathrm{SU}(2) \times \mathrm{U}(1)$ invariant couplings and conserved $B$ and $L$ numbers. This leads to the existence of 5 scalar and 5 vector LQ's with distinct SM transformation properties [76]. In general, present data constrain difermions to have masses larger than $50-150 \mathrm{GeV}$.

Leptoquarks can be produced in pairs at $e^{+} e^{-}$colliders through gauge boson exchange; significant $t$-channel quark exchange can be present in some channels if the quark-lepton-LQ couplings are not too small. Depending on the charge, the spin and isospin of the $L Q$, the cross sections can vary widely [92]. LQ's can also be pair produced in $\gamma \gamma$ collisions via $t$-channel LQ-exchange. Single production of scalar and vector $L Q$ s has been also studied in the $e^{+} e^{-}, e \gamma$ and $\gamma \gamma$ modes of the NLC [93]. The kinematical reach is thus extended to $\sim \sqrt{s}$, but the production rates are suppressed by the unknown LQ Yukawa coupling to quark-lepton pairs. At e $\gamma$ colliders, first generation LQ's are observable as long as the cor- responding squared LQ Yukawa coupling is larger than about $10^{-2} e^{2}$ (where $e^{2} \equiv 4 \pi \alpha$ is the electromagnetic coupling strength) [93].

Leptoquarks are color-nonsinglets and hence strongly interacting. As a result, LQ's can be produced at hadron colliders with very large rates. At the LHC, pair production in the $g g / q \bar{q} \rightarrow \mathrm{LQ} \overline{\mathrm{LQ}}$ process leads to cross sections ranging from a few $\mathrm{nb}$ for masses $\mathcal{O}(0.1 \mathrm{TeV})$ to a few fb for masses $\mathcal{O}(1 \mathrm{TeV})$. The rate for vector particles (which depends on their anomalous magnetic moment $\kappa)$ is substantially higher than for scalars. At the LHC with $100 \mathrm{fb}^{-1}$ the search reach for scalar/vector LQ's is $1.4 / 2.2(1.8) \mathrm{TeV}$ for $\kappa=1(0)$, if one assumes a branching fraction of unity for the $e^{+} e^{-}+$two jet final state [94]. Single scalar LQ production, through $g u \rightarrow e^{+} \mathrm{LQ}$ and $g d \rightarrow \bar{L} \mathrm{LQ}$, can also lead to large cross sections for Yukawa couplings of order unity. In this case masses up to $\sim 1.5 \mathrm{TeV}$ can be reached at the LHC [94]. Vector leptoquarks have larger production rates and the discovery reach can be extended to $\sim 2 \mathrm{TeV}$. Although LQ's can also be singly produced in association with a $W_{R}$ in alternative Left-Right models via $g u \rightarrow W_{R}+\mathrm{LQ}$, a detailed study of the signal and backgrounds has shown that the LQ discovery reach in this case is quite limited [95]. For first generation leptoquark searches, ep colliders are particularly well suited. Such LQ's can be produced as $s$ channel resonances in $e q \rightarrow \mathrm{LQ}$. For example, at $\mathrm{LEP} \times \mathrm{LHC}$ masses up to $1 \mathrm{TeV}$ can be reached, unless the corresponding LQ Yukawa couplings are very small.

Dilepton production has been considered at the NLC in the three collider modes: $e^{+} e^{-}, e \gamma$ and $\gamma \gamma$. These particles are accessible up to masses close to $\sqrt{s} / 2$ in pair production, $e^{+} e^{-} / \gamma \gamma \rightarrow X^{++} X^{--}$: the rates (especially in $\gamma \gamma$ collisions because of the charge) are very large and the signatures (four leptons) are spectacular. Dileptons can also be singly produced in the three modes of the collider [96]. Diquarks can be pair produced in $e^{+} e^{-}$and $\gamma \gamma$ collisions for masses smaller than $\sqrt{s} / 2$ with appreciable rates, with a signal consisting of an excess of 4 jet events. They can be also produced at hadron colliders in pairs (or singly for the first generation diquark if its coupling to quark pairs is not too small). However, large QCD backgrounds present a formidable challenge to diquark searches at the LHC.

\subsection{Excited Fermions}

The existence of excited particles is a characteristic signal of substructure in the fermionic sector [80]: if the known fermions are composite (composed of more elementary constituents called preons), then they should be the ground state of a rich spectrum of excited states which decay down to the former states via a magnitic dipole type de-excitation. Since there is not yet a at isfactory and predictive dynamical model for compnis- 
ite fermions, one has to use phenomenological inputs to study this scenario. For simplicity, one assumes that the excited fermions have spin and isospin $1 / 2$; their couplings to gauge bosons are vector-like (form factors and new contact interactions may also be present). Furthermore, the coupling which describes the transition between excited and ordinary fermions is taken to be chiral and inversely proportional to the compositeness scale $\Lambda$ which is of $\mathcal{O}(1 \mathrm{TeV})$.

Excited fermions can be pair-produced in $f \bar{f}$ annihilation via $s$-channel gauge boson exchange $(V=W$, $Z, \gamma$ or gluon), although some suppression due to form factors is expected [81]. The excited fermions decay into gauge bosons and their ordinary partners, $f^{\star} \rightarrow f V$. The charged leptons have the electromagnetic decay which has at least a branching ratio of $30 \%$, and the excited quarks decay most of the time into quarks and gluons. These two decays constitute a very characteristic signature and discriminate them from the exotic fermions previously discussed.

In $e^{+} e^{-}$and $\gamma \gamma$ collisions, the processes and the cross sections are the same as the ones previously described for vector-like exotic fermions (up to possible form-factor suppressions); hence the detection of $f^{*}$ masses up to the kinematic limit may be possible. Excited fermions can be singly produced with their light partners, but the rates are suppressed by a factor of $1 / \Lambda^{2}$. At $e^{+} e^{-}$colliders, for quarks and second/third generation leptons, for which the process is mediated by $s$-channel boson exchange, the cross sections are very small. But for the first generation excited fermions, one has substantial contributions due to additional $t$-channel diagrams: $W$ exchange for $\nu_{e}^{*}$ and $Z / \gamma$ exchanges for $e^{*}$, which increase the cross sections by several orders of magnitude. The excited leptons can be also singly produced in $e \gamma$ collisions $\left(e^{*}\right.$ as a resonance and $\nu^{*}$ in association with a $W$ ) with much larger rates. All excited fermions can be singly produced in $\gamma \gamma$ collisions with appreciable cross sections. For $\Lambda \sim$ few $\mathrm{TeV}$, excited quarks and leptons can easily be found in such machines, if kinematically allowed [83].

Due to the special couplings of the electron to the excited leptons of the first generation, single production of $e^{*}$ through $t$-channel $\gamma$ and $Z$ exchange, and $\nu_{e}^{*}$ through $W$ exchange are possible processes in $e p$ collisions. The cross sections are large; requiring a few tens of events to establish a signal, one can probe $\nu^{*}$ and $e^{*}$ masses up to $800 \mathrm{GeV}$ at $\mathrm{LEP} \times \mathrm{LHC}$ for $\int \mathcal{L}=2 \mathrm{fb}^{-1}$ [97]. Excited quarks of the first generation can also be produced in $e p$ collisions, however background problems make this possibility less interesting.

Excited quarks can be produced in $p p$ collisions through a variety of mechanisms [81]. The dominant production channels are the gluonic excitation of quarks $g+q \rightarrow q^{*}$ which occurs through the $q^{*} q g$ magnetic interaction, and the excitation through the preon interac- tions $q q \rightarrow q q^{*}$ and $q^{*} q^{*}$. The latter is also responsible for excited lepton production, $q \bar{q} \rightarrow e e^{*}$ and $e^{*} e^{*}$. The signatures of excited quarks are dijet mass bumps, jet + gauge boson and jet + lepton pair combinations. Excited leptons would reveal themselves in leptons + gauge particles or leptons + quark jets. QCD backgrounds have been studied and have been shown to be under control. At the LHC with a luminosity of $10 \mathrm{fb}^{-1}$, a mass range of 5-6 TeV and $\sim 4 \mathrm{TeV}$ can be reached for excited quarks and excited leptons, respectively [89].

\subsection{Synopsis}

The prospects for the discovery of new fermions (of the sequential, exotic, or excited variety) or di-fermions at future high-energy colliders can be summarized as follows:

$e^{+} e^{-}, e \gamma, \gamma \gamma, e^{-} e^{-}$colliders:

- All new particles considered in this section (except for weak isosinglet neutrinos) can be pair produced in $e^{+} e^{-}$collisions up to the kinematical limit of $\sqrt{s} / 2$. Similarly for new charged particles in $\gamma \gamma$ collisions.

- Due to their special couplings to the electron, the first generation of exotic leptons, excited leptons, leptoquarks and dileptons can be singly produced if the mixing angles, the compositeness scale or the couplings to the light fermion pairs are not prohibitively small; one can then reach masses close to the center-of-mass energy of the collider. Here, all four potential modes of the NLC can be useful.

- Due to the clean environment of these machines, one can perform precision measurements which allow one to probe the indirect effects of some of these particles for mass scales much higher than the center-of-mass energy.

ep colliders:

- $e p$ colliders are well suited for the production of the first generation exotic leptons, excited leptons and especially leptoquarks, if the mixing angles, the compositeness scale or the couplings to quark-lepton pairs, respectively, are not too small. At LEP $\times$ LHC, masses around $1 \mathrm{TeV}$ can be reached.

pp colliders:

- Proton colliders are ideal machines for the production of new color non-singlets. At the LHC, particle masses in the $\mathrm{TeV}$ range can be probed: $\sim 1$ $\mathrm{TeV}$ for sequential or exotic quarks, $\sim 1.5-2 \mathrm{TeV}$ for leptoquarks and $5-6 \mathrm{TeV}$ for excited quarks for reasonable values of the compositeness scale. 
- New color-singlet particles can also be produced, but with smaller cross sections than for quarks. However the signatures are cleaner and might compensate for the small rates. For instance, some exotic leptons can be discovered for masses smaller than $\sim 1 / 2$ $\mathrm{TeV}$; through contact interactions, excited electrons can also be produced for masses in the $\mathrm{TeV}$ range.

Finally, it should be noted that the effects of new interactions may be observable even if all the associated new particles beyond the SM are too heavy to be observed directly. If the mass scale of the new physics, $\Lambda$, is assumed to be much larger than $m_{Z}$, then one can formally integrate out the heavy particles and remove them from the full theory. The resulting low-energy effective theory will differ from the SM by new (anomalous) interactions among SM particles which are suppressed by inverse powers of $\Lambda$. For example, new physics beyond the SM could generate four-fermion contact interactions [98], which could be detected as deviations from SM predictions in 2-to-2 scattering processes involving quarks and/or leptons [91]. In this way, one may have experimental sensitivity to much larger values of $\Lambda$ as compared to direct searches for new particles. The search for anomalous couplings in the SM may be particularly fruitful in the precision study of $W$ and $Z$ self-interactions and top-quark interactions. Since these are the heaviest particles of the SM, it is plausible that their interactions could be modified by the dynamics underlying the generation of mass. The phenomenology of anomalous gauge boson and top-quark couplings is studied in more detail in the next two chapters.

\section{Anomalous Gauge Boson Couplings}

\subsection{Parametrization of Anomalous Gauge Bosons Interactions}

One of the most direct consequences of the $S U(2) \times$ $U(1)$ gauge symmetry of the SM, the non-abelian selfcouplings of the $W, Z$, and photon, remains poorly measured to date. Although information on these couplings can be extracted from low energy data and high precision measurements at the $Z$ pole, there are ambiguities and model dependencies in the results. However, a direct measurement of these vector boson couplings is possible in present and future collider experiments, in particular via pair production processes like $e^{+} e^{-} \rightarrow W^{+} W^{-}$and $p \stackrel{(-)}{p} \rightarrow W^{+} W^{-}, W \gamma, W Z$. The first and major goal of such experiments will be the verification of the SM predictions at a quantitative level.

For our discussion of experimental sensitivities we employ a parameterization of the $W W V$ couplings ( $V=$ $\gamma, Z)$ in terms of a phenomenological effective La- grangian [99]:

$$
\begin{aligned}
i \mathcal{L}_{W W V}= & g_{W W v}\left[g_{1}^{V}\left(W_{\mu \nu}^{\dagger} W^{\mu} V^{\nu}-W_{\mu}^{\dagger} V_{\nu} W^{\mu \nu}\right)\right. \\
& \left.+\kappa_{V} W_{\mu}^{\dagger} W_{\nu} V^{\mu \nu}+\frac{\lambda_{V}}{m_{W}^{2}} W_{\lambda \mu}^{\dagger} W_{\nu}^{\mu} V^{\nu \lambda}\right]
\end{aligned}
$$

Here the overall coupling constants are defined as $g_{W W \gamma}=e$ and $g_{W W Z}=e \cot \theta_{W}$. The couplings $g_{1}^{V}, \kappa_{V}$, and $\lambda_{V}$ need to be determined experimentally. Within the SM, at tree level, they are given by $g_{1}^{Z}=g_{1}^{\gamma}=\kappa_{Z}=$ $\kappa_{\gamma}=1, \lambda_{z}=\lambda_{\gamma}=0 . g_{1}^{\gamma}=1$ is fixed by electromagnetic gauge invariance; $g_{1}^{Z}$, however, may well be different from its SM value 1 and appears at the same level as $\kappa_{\gamma}$ or $\kappa_{Z}$.

The effective Lagrangian of Eq. (9.1) parameterizes the most general Lorentz invariant and $C$ and $P$ conserving $W W V$ vertex which can be observed in processes where the vector bosons couple to effectively massless fermions. Terms with higher derivatives are equivalent to a dependence of the couplings on the vector boson momenta and thus merely lead to a form-factor behavior of these couplings [99]. Analogous to the general $W W V$ vertex it is possible to parameterize anomalous $Z \gamma V, V=\gamma, Z$ couplings in terms of two coupling constants, $h_{3}^{V}$ and $h_{4}^{V}$, if CP invariance is imposed. All $Z \gamma V$ couplings are $C$ odd; $h_{\mathfrak{Y}}^{V}$ corresponds to a dimension 6 , $h_{4}^{V}$ to a dimension 8 operator [99].

Tree level unitarity uniquely restricts the $W W V$ and $Z \gamma V$ couplings to their SM gauge theory values at asymptotically high energies. This implies that deviations of $g_{1}^{V}, \kappa_{V}, \lambda_{V}$ and $h_{i}^{V}, i=3,4$ must be parametrized by form factors which indicates that the effective Lagrangian describing the anomalous vector boson self-interactions breaks down at some high energy scale. SM one-loop effects lead to corrections of $\mathcal{O}(\alpha)$ with a form factor scale, $\Lambda_{F F}$, of order the electroweak symmetry breaking scale.

Models which have been considered so far, such as technicolor or supersymmetric models, predict rather small deviations from the SM values, of $\mathcal{O}\left(10^{-2}\right)$ or less. If we regard the Standard Model as an effective field theory, then new physics can be parameterized in a model independent way by additional gauge-invariant operators. Regardless of whether Higgs physics is strongly or weakly interacting, there are restrictions on the strength of such contributions which, however, depend on naturalness assumptions. Detailed analyses $[100,101]$ show that these restrictions leave a slim chance only that new physics will be discovered through anomalous couplings at machines which are less capable than the LHC or NLC.

\subsection{Search for Anomalous Gauge Boson Couplings at Present and Future Colliders}

The direct measurement of $W W V$ and $Z \gamma V$ couplings in present and future collider experiments in general makes 
use of the high energy behavior of the non-standard contributions to the helicity amplitudes which grow like a power of the parton center of mass energy. In di-boson production, this leads to an excess of events at large $W$, $Z$ and photon transverse momenta as well as at large invariant masses of the di-boson system. Furthermore, the various non-standard couplings contribute to different helicity amplitudes in the high energy limit. Angular distributions of the $W$ and $Z$-boson decay products, therefore, are helpful as polarization analyzers, in particular in $e^{+} e^{-}$collisions. Quantitative bounds on $\Delta g_{1}^{Z}=g_{1}^{Z}-1, \Delta \kappa_{V}=\kappa_{V}-1$ and $\lambda_{V}$ are obtained by comparing the shape of the measured and the predicted distributions.

Present and future 95\% confidence level (CL) limits on a variety of $W W V$ and $Z \gamma V$ couplings are listed in Table 11. In addition, the limits on the couplings $\Delta \kappa_{\gamma}$ and $\lambda_{\gamma}$ are displayed in bargraph form in Fig. 5. The present limits are from di-boson production at the Tevatron [102] and from single photon production at LEP [103]. The future limits are estimated for experiments at the Tevatron, LEP II, the LHC and a future linear $e^{+} e^{-}$collider (NLC). In contrast to the bounds on anomalous $W W V$ couplings, the limits on the $Z_{\gamma} V$ couplings $h_{i 0}^{V}, i=3,4$ depend significantly on the form factor scale $\Lambda_{F F}$. Here they are assumed to appear in the form $h_{i}^{V}=h_{i 0}^{V} /\left(1+\hat{s} / \Lambda_{F F}^{2}\right)^{n_{i}}, i=3,4$ with $n_{3}=3$ and $n_{4}=4$.

A direct measurement of the $W W \gamma$ vertex is achieved via $W \gamma$ production. The best current bounds on $W W V$ couplings, however, depend significantly on information obtained from $W^{+} W^{-}$and $W Z$ production at the Tevatron in the channel $p \bar{p} \rightarrow W^{+} W^{-}, W^{ \pm} Z \rightarrow$ $\ell^{ \pm} \nu j$. In deriving these bounds, $\Delta \kappa_{\gamma}=\Delta \kappa_{Z}$ and $\lambda_{\gamma}=\lambda_{Z}$ is assumed.

The present limits on anomalous $W W V$ and $Z \gamma V$ couplings can be improved by one order of magnitude or more in future Tevatron experiments if an integrated luminosity of $1 \mathrm{fb}^{-1}$ can be achieved. Raising the integrated luminosity to $10 \mathrm{fb}^{-1}$ typically leads to a factor $\sim 2$ improvement over the limits which can be achieved with $1 \mathrm{fb}^{-1}$. Fitting several different distributions simultaneously might lead to further improvements. Bounds on $\Delta g_{1}^{Z}$ and $\Delta \kappa_{V}$ are expected to be comparable to those which can be obtained at LEP II. For $\lambda_{V}$, the limits from future Tevatron experiments should be better.

The future bounds listed in Table 11 for $W^{+} W^{-}$ and $W^{ \pm} Z$ final states were obtained assuming $\lambda_{Z}=\lambda_{\gamma}$, $\Delta g_{1}^{Z}=\Delta \kappa_{\gamma} / 2 \cos ^{2} \theta_{\mathrm{w}}$ and $\Delta g_{1}^{Z}+\Delta \kappa_{Z}=\Delta \kappa_{\gamma}$. These relations are motivated by an effective Lagrangian based on a complete set of $S U(2) \times U(1)$ invariant operators of dimension 6 [101]. If one assumes $\kappa_{\gamma}=\kappa_{Z}, \lambda_{\gamma}=\lambda_{Z}$ and $\Delta g_{1}^{Z}=0$ instead, the limit on $\Delta \kappa_{\gamma}$ can be improved by about $40 \%$.

At the LHC, with an integrated luminosity of
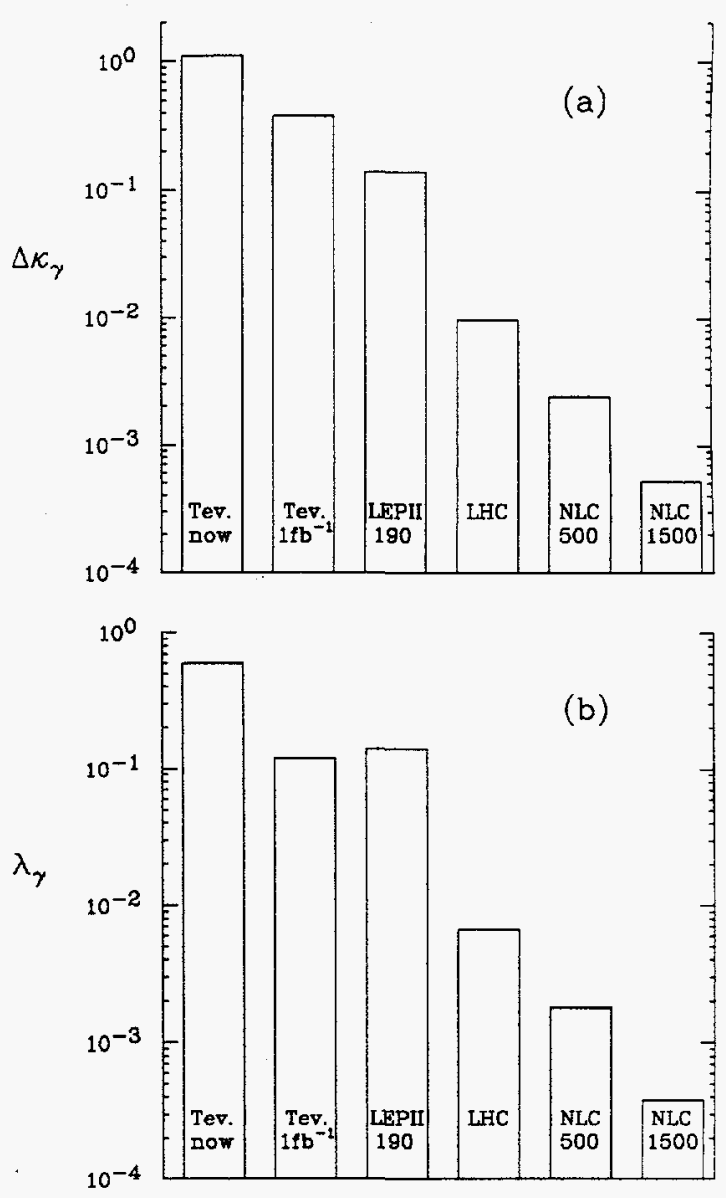

Figure 5: Present and future 95\% CL bounds on $\Delta \kappa_{\gamma}$ (a) and $\lambda_{\gamma}(\mathbf{b})$.

$100 \mathrm{fb}^{-1}$, values of $\Delta \kappa_{Z}, \Delta g_{1}^{Z}$ and $\lambda_{Z}$ of $\mathcal{O}\left(10^{-2}\right)$ can be probed. Similar sensitivities can be achieved for the $W W \gamma$ couplings. A linear $e^{+} e^{-}$collider with a center of mass energy of $500 \mathrm{GeV}$ or more in general can probe anomalous couplings of order $10^{-3}[104,50]$.

The present bounds on $Z_{\gamma} V$ couplings can be improved only by about a factor 2 at best in $Z \gamma$ production at LEP II. Future experiments at the Tevatron will be sensitive to values of $h_{30}^{V} \sim 0.11$ and $h_{40}^{V} \sim 0.006$ (with $\left.\Lambda_{F F}=1.5 \mathrm{TeV}\right)$.

Within the next few years experiments at the Tevatron and at LEP are expected to confirm the non-abelian self-couplings of the SM at the $10 \%$ level. The $\mathcal{O}(\alpha)$ deviations predicted by the $\mathrm{SM}$ and most models of new physics, however, can only be probed at the LHC or a high energy linear $e^{+} e^{-}$collider. Of course, if the scale of new physics is directly accessible at these machines one would rather focus on the spectrum of new particles and 
Table 11: Present and future $95 \% \mathrm{CL}$ bounds on $W W V$ and $Z \gamma V$ couplings.

\begin{tabular}{|c|c|c|}
\hline Experiment & Channel & Limit \\
\hline $\begin{array}{l}\mathrm{D} \emptyset \\
\text { (present limit) }\end{array}$ & $\begin{array}{c}p \bar{p} \rightarrow W^{ \pm} \gamma \rightarrow \ell^{ \pm} \nu \gamma \\
\ell=e, \mu\end{array}$ & $\begin{array}{c}-1.6<\Delta \kappa_{\gamma}<1.8 \\
-0.6<\lambda_{\gamma}<0.6\end{array}$ \\
\hline $\begin{array}{l}\text { CDF } \\
\text { (present limit) }\end{array}$ & $\begin{array}{c}p \bar{p} \rightarrow W^{+} W^{-}, W^{ \pm} Z \rightarrow \ell^{ \pm} \nu j j \\
\kappa_{\gamma}=\kappa_{Z}, \lambda_{\gamma}=\lambda_{Z}, g_{1}^{Z}=1\end{array}$ & $\begin{array}{c}-1.0<\Delta \kappa_{V}<1.1 \\
-0.8<\lambda_{V}<0.8\end{array}$ \\
\hline $\begin{array}{l}\mathrm{D} \emptyset \\
\text { (present limit) }\end{array}$ & $\begin{array}{c}p \bar{p} \rightarrow Z_{\gamma} \rightarrow \ell^{+} \ell^{-} \gamma \\
\ell=e, \mu, \Lambda_{F F}=0.5 \mathrm{TeV}\end{array}$ & $\begin{array}{l}-1.9<h_{30}^{V}<1.8 \\
-0.5<h_{40}^{V}<0.5\end{array}$ \\
\hline $\begin{array}{l}\text { L3 } \\
\text { (present limit) }\end{array}$ & $e^{+} e^{-} \rightarrow \bar{\nu} \nu \gamma$ & $\begin{array}{l}-0.9<h_{30}^{Z}<0.9 \\
-2.3<h_{40}^{Z}<2.3\end{array}$ \\
\hline $\begin{array}{l}\text { Tevatron } \\
\text { (CDF sim.) }\end{array}$ & $\begin{array}{c}p \bar{p} \rightarrow W^{ \pm} \gamma \rightarrow e^{ \pm} \nu \gamma \\
\sqrt{s}=2 \mathrm{TeV}, \int \mathcal{L} d t=1 \mathrm{fb}^{-1}\end{array}$ & $\begin{array}{c}-0.38<\Delta \kappa_{\gamma}<0.38 \\
-0.12<\lambda_{\gamma}<0.12\end{array}$ \\
\hline $\begin{array}{l}\text { Tevatron } \\
\text { (CDF sim.) }\end{array}$ & $\begin{array}{c}p \bar{p} \rightarrow W^{+} W^{-}, W^{ \pm} Z \rightarrow \ell^{ \pm} \nu j j, \ell^{+} \ell^{-} j j \\
\int \mathcal{L} d t=1 \mathrm{fb}^{-1}, \Lambda_{F F}=2 \mathrm{TeV}\end{array}$ & $\begin{array}{c}-0.31<\Delta \kappa_{\gamma}<0.41 \\
-0.19<\lambda_{\gamma}<0.19\end{array}$ \\
\hline $\begin{array}{l}\text { Tevatron } \\
\text { (NLO theor.) }\end{array}$ & $\begin{array}{c}p \bar{p} \rightarrow W^{ \pm} Z \rightarrow \ell_{1}^{ \pm} \nu_{1} \ell_{2}^{+} \ell_{2}^{-} \\
\int \mathcal{L} d t=1 \mathrm{fb}^{-1}, \Lambda_{F F}=1 \mathrm{TeV}\end{array}$ & $\begin{array}{c}-0.26<\Delta \kappa_{Z}<0.70 \\
-0.24<\lambda_{Z}<0.32\end{array}$ \\
\hline $\begin{array}{l}\text { Tevatron } \\
\text { (CDF sim.) }\end{array}$ & $\begin{array}{c}p \bar{p} \rightarrow Z \gamma \rightarrow e^{+} e^{-\gamma} \\
\int \mathcal{L} d t=1 \mathrm{fb}^{-1}, \Lambda_{F F}=1.5 \mathrm{TeV}\end{array}$ & $\begin{aligned}-0.105 & <h_{30}^{V}<0.105 \\
-0.0064 & <h_{40}^{V}<0.0064\end{aligned}$ \\
\hline $\begin{array}{l}\text { Tevatron } \\
\text { (CDF sim.) }\end{array}$ & $\begin{array}{c}p \bar{p} \rightarrow Z \gamma \rightarrow e^{+} e^{-} \gamma \\
\int \mathcal{L} d t=10 \mathrm{fb}^{-1}, \Lambda_{F F}=1.5 \mathrm{TeV}\end{array}$ & $\begin{aligned}-0.044 & <h_{30}^{V}<0.044 \\
-0.0025 & <h_{40}^{V}<0.0025\end{aligned}$ \\
\hline $\begin{array}{l}\text { LEP II } \\
(\text { L3 sim.) }\end{array}$ & $\begin{array}{c}e^{+} e^{-} \rightarrow W^{+} W^{-} \rightarrow \ell^{ \pm} \nu j j \\
\sqrt{s}=190 \mathrm{GeV}, \int \mathcal{L} d t=500 \mathrm{pb}^{-1}\end{array}$ & $\begin{array}{c}-0.13<\Delta \kappa_{\gamma}<0.14 \\
-0.13<\lambda_{\gamma}<0.14\end{array}$ \\
\hline $\begin{array}{l}\text { LEP II } \\
(\mathrm{L} 3 \mathrm{sim} .)\end{array}$ & $\begin{array}{c}e^{+} e^{-} \rightarrow Z_{\gamma} \rightarrow \nu \bar{\nu} \gamma, \sqrt{s}=180 \mathrm{GeV} \\
\int \mathcal{L} d t=500 \mathrm{pb}^{-1}, \Lambda_{F F}=1 \mathrm{TeV}\end{array}$ & $\begin{array}{l}-0.50<h_{30}^{Z}<0.50 \\
-0.45<h_{40}^{Z}<0.45\end{array}$ \\
\hline $\begin{array}{l}\text { LHC } \\
\text { (NLO theor.) }\end{array}$ & $\begin{array}{c}p p \rightarrow W^{ \pm} Z \rightarrow \ell_{1}^{ \pm} \nu_{1} \ell_{2}^{+} \ell_{2}^{-} \\
\int \mathcal{L} d t=100 \mathrm{fb}^{-1}, \Lambda_{F F}=3 \mathrm{TeV}\end{array}$ & $\begin{array}{c}-0.006<\Delta \kappa_{Z}<0.0097 \\
-5.3 \cdot 10^{-3}<\lambda_{Z}<6.7 \cdot 10^{-3}\end{array}$ \\
\hline $\begin{array}{l}\text { LHC } \\
\text { (LO theor.) }\end{array}$ & $\begin{array}{c}p p \rightarrow Z \gamma \rightarrow e^{+} e^{-} \gamma \\
\int \mathcal{L} d t=10 \mathrm{fb}^{-1}, \Lambda_{F F}=1.5 \mathrm{TeV}\end{array}$ & $\begin{array}{l}-5.1 \cdot 10^{-3}<h_{30}^{V}<5.1 \cdot 10^{-3} \\
-9.2 \cdot 10^{-5}<h_{40}^{V}<9.2 \cdot 10^{-5}\end{array}$ \\
\hline NLC & $\begin{array}{c}e^{+} e^{-} \rightarrow W^{+} W^{-} \rightarrow \ell^{ \pm} \nu j j \\
\sqrt{s}=500 \mathrm{GeV}, \int \mathcal{L} d t=80 \mathrm{fb}^{-1}\end{array}$ & $\begin{array}{c}-0.0024<\Delta \kappa_{\gamma}<0.0024 \\
-0.0018<\lambda_{\gamma}<0.0018\end{array}$ \\
\hline NLC & $\begin{array}{c}e^{+} e^{-} \rightarrow W^{+} W^{-} \rightarrow \ell^{ \pm} \nu j j \\
\sqrt{s}=1500 \mathrm{GeV}, \int \mathcal{L} d t=190 \mathrm{fb}^{-1}\end{array}$ & $\begin{array}{c}-5.2 \cdot 10^{-4}<\Delta \kappa_{\gamma}<5.2 \cdot 10^{-4} \\
-3.8 \cdot 10^{-4}<\lambda_{\gamma}<3.8 \cdot 10^{-4}\end{array}$ \\
\hline
\end{tabular}


direct measurements of their interactions with the gauge boson sector.

\section{Top Quarks as a Window to Electroweak Symmetry Breaking}

Among the various matter fermions, the top quark must be considered the most mysterious. It is the heaviest known elementary particle, with mass 30-40 times that of the next heaviest quark. It is more strongly coupled to the mechanism of electroweak symmetry breaking than the weak gauge bosons themselves. It is plausible, in fact, that the top quark is an essential component of this mechanism. On a more mundane level, the top quark is the only quark or lepton whose couplings to the Standard Model are not constrained to percent accuracy by the LEP experiments. Thus, the couplings of the top can easily yield surprises.

Most of this section is concerned with the search for anomalies in the couplings of the top quark to the Standard Model gauge bosons. We will consider first the possibility of large deviations from the standard properties of the top quark and then the precision experiments that could show signs of smaller deviations. Finally, we will discuss the possibility that the top quark has decay modes outside the Standard Model.

\subsection{Anomalous Top Couplings - s-channel Resonance}

The original CDF announcement of evidence for the top quark $[105,106]$ came with an anomaly, the measurement of a production cross section 2-3 times larger than the theoretical prediction. The more recent announcements of the observation of the top quark by CDF [107] and D0 [108] give a production cross section of $6.5 \pm 1.8 \mathrm{pb}$, which is consistent with the Standard Model unless the top quark mass is above $185 \mathrm{GeV}$. Nevertheless, the possibility of order-1 deviations of the top quark couplings from the predictions of the Standard Model is extremely tantalizing and might well open a new window into physics of flavor.

At the Tevatron collider, the dominant mechanism of $t \bar{t}$ production is through $q \bar{q}$ annihilation; at a top mass of $175 \mathrm{GeV}$, the $g g$ fusion cross section is a factor 10 smaller. To dramatically enhance this cross-section, it is natural to consider models with an $s$-channel resonance in $t \bar{t}$ production, with a mass of $500-600 \mathrm{GeV}$, just above the $t \bar{t}$ threshold. Two groups of authors have proposed explanations of this type based on pre-existing theoretical models $[109,66]$.

Higher luminosity running at the Tevatron collider will clarify this situation. The clearest prediction of the model is an excess of events at large $t \bar{t}$ invarinant mass and high $t$ transverse momentum. Taking the model [109] with a $600 \mathrm{GeV}$ resonance for definiteness, event samples of $0.1 \mathrm{fb}^{-1}, 3 \mathrm{fb}^{-1}$, and $100 \mathrm{fb}^{-1}$ lead to roughly 10,300 , and 10,000 observed $t \bar{t}$ events with $m(t \bar{t})>600$ $\mathrm{GeV}$, assuming efficiency (1/10) for detection, above a background 5 times lower. These samples correspond to the goals for the current Tevatron collider run, the new Main Injector upgrade, and the $\mathrm{TeV}^{*}$ luminosity upgrade. With the same event samples, one could put lower limits on the mass of an $s$-channel resonance at roughly $700,1000,1500 \mathrm{GeV}$.

At the LHC, the production cross section for $t \bar{t}$ is $90 \% g g$ fusion, but the observed rates are large, of order $10^{6} t \bar{t}$ pairs per year. The cross section measurement at the LHC clearly distinguishes the $q \bar{q}$ from the $g g$ model for the $s$-channel resonance; these models predict cross sections differing by a factor of 10 . The mass reach of the LHC with $10 \mathrm{fb}^{-1}$ is about $1000 \mathrm{GeV}$ in the $q \bar{q}$ model and $2000 \mathrm{GeV}$ in the $g g$ model, with stronger constraints at $100 \mathrm{fb}^{-1}$

Since both models involve resonances that do not couple to leptons, their direct effect on $t \bar{t}$ production is not easily tested at an $e^{+} e^{-}$collider. However, for both models, an $e^{+} e^{-}$collider with an energy about $1 \mathrm{TeV}$ could provide important complementary information on the associated production of the new resonances with $t \bar{t}$ and on the pair-production of related new particles with electric charge and exotic color.

\subsection{Anomalous Top Couplings-Effective Lagrangian}

If the top quark production cross section proves not to be anomalous at the zeroth order, how well can the top quark couplings be searched for anomalies at higher order? An appropriate framework for discussing this question is an effective Lagrangian governing the top quark coupling to gauge bosons. For example, the coupling of the $W$ boson to $t \bar{b}$ may be written

$$
\Delta \mathcal{L}=\frac{g}{\sqrt{2}} F_{1}^{W}\left(q^{2}\right) \bar{t} \gamma^{\mu} b_{L} W_{\mu}+\frac{1}{4 m_{t}} F_{2}^{W}\left(q^{2}\right) \bar{t} \sigma^{\mu \nu} b_{L} F_{\mu \nu}^{W} .
$$

Analogous formulae define the $F_{1}$ and $F_{2}$ form factors of $g, \gamma$, and $Z$. Phenomenological couplings of this form have been studied by many authors [110]. Electroweak interactions typically give contributions to these form factors at the $1 \%$ level or below. However, it is likely that there are larger contributions from models in which the top quark is strongly coupled to the symmetry breaking sector. For example, extended technicolor models give contributions to the various $F_{1}$ form factors of $t_{L}, t_{R}$, and $b_{L}$ which are typically of order $5 \%$ but whose pattern depends on the details of the model [111].

We consider first the measurement of these form factors in hadron-hadron collisions. It is possible that these form factor corrections are large; in fact, it has been proposed [112] that a nonzero color anomalous magnetic mo- 
ment $F_{2}^{g} \sim 0.25$ could provide an alternative explanation of the large cross section reported by CDF. On the other hand, if the form factor effect is smaller, one's ability to measure it quickly becomes limited by the $15 \%$ QCD uncertainty in the $t \bar{t}$ production cross section. In a oneparameter fit based on the total cross section, the Tevatron could bound the $F_{2}$ of the $t t g$ coupling to \pm 0.15 (95\% conf.) with $0.1 \mathrm{fb}^{-1}$ and reach the systematics limit of \pm 0.10 with $0.5 \mathrm{fb}^{-1}$ [113]. The same systematics limit appears at the LHC. Yuan has suggested that the $F_{1}$ coefficients of the $W t \bar{b}$ couplings can be constrained by measuring the cross section for single top production: $g W \rightarrow t \bar{b}$; this cross section, however, has a theoretical uncertainty of order $30 \%$ [114]. One parameter of $t \bar{t}$ production at hadron colliders which seems very promising for a precision constraint is the measurement of the longitudinal/transverse polarization of $W$ bosons from top decay; a $3 \mathrm{fb}^{-1}$ event sample at the Tevatron should bring the statistical uncertainty below $4 \%$ [115].

The environment offered by $e^{+} e^{-}$colliders offers many advantages for a precision study of the top couplings. In addition to low backgrounds and the ability to reconstruct $t \bar{t}$ in the 6 -jet mode, top production is characterized by order-1 forward-backward and polarization asymmetries which reflect sizeable interference of the $\gamma$ and $Z$ exchange diagrams. Top quarks produced in the forward direction carry the electron polarization and thus provide a polarized sample for studies of the decay form factors.

For comparison with the estimate given above, the total cross section measurement at an $e^{+} e^{-}$collider with $20 \mathrm{fb}^{-1}$ should bound a common $F_{2}$ for the $t t \gamma$ and $t \bar{t} Z$ couplings, in a one-parameter fit, at \pm 0.03 . However, much more detailed studies are possible, at least with large event samples. For example, consider a variation in the $Z t \bar{t}$ coupling leaving the $t \bar{t} \gamma$ coupling fixed. The left- and right-handed form factors of $Z t \bar{t}$ coupling can be constrained in a two-parameter fit for an event sample of $100 \mathrm{fb}^{-1}$ at $500 \mathrm{GeV}$, to deviations of \pm 0.08 (95\% conf.). (The systematic contribution to this error should be small, and will be analyzed carefully for the final report.) For comparison with the parameter of top decays discussed above, Fujii has found that the fraction of longitudinal $W$ bosons in $t$ decay can be determine with a statistical precision of $2 \%$ with $10 \mathrm{fb}^{-1}$ of data at 350 $\mathrm{GeV}$. By studying top pair production with an extra jet, one can obtain [116] a direct limit on the color anomalous magnetic moment $F_{2}^{g}$, to be compared with that from hadron colliders; with $200 \mathrm{fb}^{-1}$, the constraint is \pm 0.16 ( $95 \%$ conf.).

The $F_{1}$ associated with the $W t \bar{b}$ vertex can be obtained from a measurement of the total width of the top quark, assuming that no exotic decay modes are seen. This quantity can be measured at the threshold for $t \bar{t}$ production by three techniques: the width of the $1 \mathrm{~S}$ reso- nance or shoulder, the momentum distribution of reconstructed top quarks decaying to $W b$, and the forwardbackward asymmetry of top production due to the interference of overlapping $S$ and $P$ wave states. Fujii, Matsui, and Sumino [117] have found that, by combining these techniques, the width of the $t$ can be determined with $100 \mathrm{fb}^{-1}$ of data to an accuracy of $4 \%$, plus an error from the uncertainty in $\alpha_{s}$ that boost this number to about $10 \%$. We should also note that, in models with a relatively light Higgs boson, of mass about $100 \mathrm{GeV}$, the height of the $1 \mathrm{~S}$ enhancement at the $t \bar{t}$ threshold measures the $t \bar{t}$ Higgs coupling constant, to about $25 \%$ accuracy.

In addition to magnetic dipole $\left(F_{2}\right)$ couplings, the top quark may also have electric dipole couplings which violate CP. Direct measurements of the form factors give limits on the CP-violating couplings similar to those cited above. This is probably not adequate for those models of $\mathrm{CP}$ violation involving $\mathrm{CP}$-violating Higgs bosons coupling strongly to the top quark. However, Schmidt and Peskin have suggested observing the energy asymmetry in $e^{+}$vs. $e^{-}$decay products of $t \bar{t}$. Combined with the high statistics available at the LHC, this technique could reasonable reach the $\mathrm{CP}$ asymmetries of $10^{-3}-10^{-4}$ predicted by the models [118].

\subsection{Exotic Decays of the Top Quark}

The top quark may also connect to exotic states by its decays. Many exotic decay modes of top have been considered in the literature. Here are the most important ones:

$t \rightarrow H^{+} b$ : This mode is interesting because it is the best way to find $H^{+}$at hadron colliders. In addition, the ratio of $t$-quark branching ratios to $H^{+} b$ and $W^{+} b$ depends on $\tan \beta$, providing one of the best ways to measure this parameter of an extended Higgs sector. The decay mode should be found at LHC with $10^{33}$ luminosity, or with a $500 \mathrm{GeV} e^{+} e^{-}$collider with $1 \mathrm{fb}^{-1}$ of data.

$t \rightarrow \tilde{\chi}^{0} \tilde{t}$ : This is a supersymmetric decay of top which occurs in a reasonable volume of parameter space, with a branching ratio of roughly $5 \%$ if $\widetilde{t}$ is much lighter than $t[119]$.

$t \rightarrow c h^{0}, t \rightarrow c Z^{0}, t \rightarrow c g$, etc.: These flavor changing neutral current modes are extremely rare in the Standard Model, with branching ratios $<10^{-10}$. If these modes are dramatically enhanced by new physics, they might be observed at the LHC.

$t \rightarrow s W^{+}$. This mode is expected to be at the $0.1 \%$ level in the Standard Model. If this mode is enhanced to the few-percent level, it should be observable at an $e^{+} e^{-}$ collider [120]. 


\section{Virtual Effects of New Physics}

\subsection{Overview}

The study of virtual effects can open an important window on electroweak symmetry breaking and physics beyond the Standard Model (SM). The examination of indirect effects of new physics in higher order processes offers a complementary approach to the search for direct production of new particles at high energy accelerators. In fact, tests of loop induced couplings can provide a means of probing the detailed structure of the SM at the level of radiative corrections where Glashow-Iliopoulos-Maiani (GIM) cancellations are important. In some cases the constraints on new degrees of freedom via indirect effects surpass those obtainable from collider searches. In other cases, entire classes of models are found to be incompatible. Given the large amount of high luminosity data which will become available during the next decade, this approach to searching for physics beyond the SM will become a valuable tool.

\subsection{Precision Electroweak Measurements}

Virtual effects from new physics are easily detected in precision electroweak measurements. These include the measurements of the $Z$ line-shape and asymmetries at SLD/LEP, deep inelastic muon-neutrino scattering, and atomic parity violation. Because of the accurate theoretical predictions in the electroweak sector of the SM, especially in purely leptonic processes that are free of QCD corrections, any observed deviations must be explained as due to new physics not encompassed within the SM.

Radiative corrections from new physics are often classified into two groups; the 'oblique' and the 'direct' corrections. 'Oblique' refers to the vacuum polarization corrections to the gauge boson propagators. They are independent of the external particles and thus are universal to all electroweak processes. 'Direct' refers to the vertex and box corrections which are specific to each process. Because of the universality of oblique corrections, it is possible to express them in a model independent fashion with a few phenomenological parameters. Under the two assumptions that the electroweak gauge group is the standard $S U(2)_{L} \times U(1)_{Y}$, and that the scale of new physics is large compared to the electroweak scale, the virtual effects of new physics can be expressed in terms of 3 parameters, often called $S, T$, and $U$ [121]. Using a set of measurements to which the direct corrections from new physics are expected to be small, one can place tight constraints on the values of $S$ and $T$. These include the purely leptonic processes at SLD/LEP, and atomic parity violation measurements. Currently, the strongest constraint on $S$ and $T$ comes from the SLD/LEP measurement of $\sin ^{2} \theta_{w}^{\text {eff }}$ and $\Gamma\left(Z \rightarrow \ell^{+} \ell^{-}\right)$.
If one relaxes the second assumption that the scale of new physics is large compared to the electroweak scale, then it becomes necessary to introduce three additional parameters called $V, W$, and $X$ [122]. If one relaxes the first assumption and extends the electroweak gauge group to $S U(2)_{L} \times U(1)_{Y} \times U(1)_{Y^{\prime}}, S U(2)_{L} \times S U(2)_{R} \times$ $U(1)_{B-L}, S U(3) \times U(1)$, etc. then it becomes necessary to take into account the exchange of the additional gauge bosons as well as their virtual loop effects so these models cannot be treated within the $S-T$ formalism and each case must be analyzed separately.

A process where the direct corrections from new physics is expected to be sizable is $Z \rightarrow b \bar{b}$. This is due to the fact that the $b$-quark is the isospin partner of the $t$-quark so the same mechanism which generates the large $t$-quark mass will also generate a large vertex correction to this process. This is true in the SM which has a vertex correction proportional to $m_{t}^{2}$, and also in Technicolor models and SUSY models. If such new vertex corrections exist, then one should be able to see a deviation from the SM prediction after the universal oblique correction effects have been folded out. Such a deviation at the $2 \sigma$-level is presently observed at LEP in the ratio $R_{b}=\Gamma_{b \bar{b}} / \Gamma_{\text {had }}$.

\subsection{Rare Processes in the Quark Sector}

Rare $K$ processes have played a strong and historical role in constraining new interactions. The box and penguin diagrams which typically mediate these processes receive contributions from the $\mathrm{SM}$, as well as potentially sizeable contributions from new physics. For example, the strongest limit (albeit assumption dependent) on the mass of a right-handed $W$-boson is derived from its contributions to $K^{0}-\bar{K}^{0}$ mixing, and the requirement of near degeneracy of squark masses results from a super-GIM mechanism imposed in the $K$ sector. Rare $K$ decay experiments at Brookhaven and KTEV at the Tevatron will provide further probes in the near future. The decay $K^{+} \rightarrow \pi^{+} \nu \bar{\nu}$ is an interesting mode as it is theoretically clean and experiment will reach the SM level during the next decade. $K_{L}^{0} \rightarrow \mu^{ \pm} e^{\mp}$ is forbidden in the SM and provides a probe of lepton number violation, leptoquark exchange, and family symmetries.

Short distance SM contributions to rare charm processes are small due to the effectiveness of the GIM mechanism (as there are no large quark mass splittings in the loops). Long distance effects usually dominate and are difficult to reliably calculate. Observation of $D \rightarrow \rho \gamma$ would provide an excellent test of the techniques used in these calculations, and could be used to reliably evaluate the related long distance contributions to $B \rightarrow K^{*} \gamma, \rho \gamma$. However, there is a window for the clean observation of possible new physics contributions in some processes, such as $D^{0}-\bar{D}^{0}$ mixing, $D \rightarrow X_{u} \ell^{+} \ell^{-}$, 
and $D \rightarrow \mu \mu$. The types of non-standard scenarios which give the largest contributions to these reactions are (i) two-Higgs-doublet models in the large $\tan \beta$ region, (ii) multi-Higgs doublet models with flavor changing couplings (iii) new $Q=-1 / 3$ quarks, (iv) supersymmetric models with non-degenerate squarks, and (v) variants of left-right symmetric models. Several high volume charm experiments are planned for the future, with $10^{7-8}$ charm mesons expected to be reconstructed. $D^{0}-\bar{D}^{0}$ mixing can then be probed another 1-2 orders of magnitude below present sensitivities.

A large amount of data in the $B$-meson system will be acquired during the next decade at SLC/LEP, CESR, the Tevatron, and the SLAC and KEK B-factories. Here, one-loop processes occur with sizeable rates in the SM, due to large top-quark contributions. Many classes of new models can give significant and testable contributions to rare $B$ processes. The inclusive process $B \rightarrow X_{s} \gamma$ and the related exclusive decay $B \rightarrow K^{*} \gamma$ has been observed by CLEO and is the first direct observation of a penguin mediated process. It has provided strong restrictions on parameters in several theories beyond the SM [123], with the bounds being competitive with those from direct collider searches. For example, it constrains the mass of a charged Higgs boson to be $>260 \mathrm{GeV}$ for $m_{t}=175 \mathrm{GeV}$, and provides complementary bounds to those obtained at colliders on an anomalous $W W \gamma$ vertex. The inclusive processes $b \rightarrow s \ell \ell, s \nu \bar{\nu}$ are also excellent probes of new physics. They are sensitive to the form of possible new interactions since they allow measurements of various kinematic distributions as well as the total rate. Techni-GIM models predict $B(b \rightarrow$ $\left.s \mu^{+} \mu^{-}\right) \sim \mathcal{O}\left(10^{-4}\right)$ and appear to already be in conflict with the present experimental limits.

$\mathrm{CP}$ violation in the $B$ system will be examined at the SLAC and KEK B-Factories. Signals for new sources of $\mathrm{CP}$ violation include, (i) non-closure of the 3 generation unitarity triangle, (ii) non-vanishing CP asymmetries for the channels $B_{d}^{0} \rightarrow \phi \pi^{0}, K_{S}^{0} K_{S}^{0}$, (iii) inconsistency of separate measurements of the angles of the unitarity triangle, and (iv) a deviation of CP rates from SM predictions. The large data sample and asymmetric configuration of the B-factories will provide a series of unique consistency tests of the quark sector and will challenge the SM in a new and quantitatively precise manner.

Loop-induced top-quark decays are small in the SM due to the effectiveness of the GIM mechanism. Branching fractions for $t \rightarrow c \gamma, c Z, c g, c h$ lie in the range $10^{-12}$ $10^{-8}$. Long distance effects are expected to be negligible. Contributions from new physics can enhance these rates by 3-4 orders of magnitude. The tree-level decay $t \rightarrow c h$, if kinematically accessible, is possible in multi-higgs models without natural flavor conservation. $t \rightarrow \tilde{t} \tilde{\chi}^{0}$ can occur in SUSY if the stop-squark is light. These would provide clean signals for new physics and may be observable at a high luminosity Tevatron.

\subsection{Electric Dipole Moments}

Electric dipole moments (EDMs) of atoms, molecules, and the neutron are the most sensitive test of low energy flavor conserving CP violation. Such CP violation from the CKM angle is highly suppressed (by at least 3 loops), while other sources of $\mathrm{CP}$ violation are generally not so suppressed. Atoms with an unpaired electron (such as ${ }^{133} \mathrm{Cs}$ ) are generally most sensitive to the electron EDM, while the neutron and atoms with paired electrons (such as ${ }^{199} \mathrm{Hg}$ ) are sensitive to $\mathrm{CP}$ violation in the strongly interacting sector. Although no EDM has yet been observed, the current bounds already put stringent constraints on CP-violating extentions of the SM. For example in supersymmetric theories the bound of $1.3 \times 10^{-27} \mathrm{e} \mathrm{cm}$ for the EDM of ${ }^{199} \mathrm{Hg}$ places a limit on new CP-violating phases that can arise in gaugino mass terms and in off-diagonal squark masses and squark interactions. These new phases must be less than a few tenths of a percent for $M_{\text {SUSY }} \simeq 100 \mathrm{GeV}$. This bound also limits the CP-violating phases in the Higgs potential in multi-Higgs theories to be less than roughly $10^{-1}$ for $m_{\text {Higgs }} \simeq 100 \mathrm{GeV}$. The bound from the neutron gives a slightly less stringent limit. Improvement in most experiments is anticipated over the next few years. New techniques, such as atomic traps, may allow improvement of up to a few orders of magnitude. If positive measurements are eventually made the QCD vacuum angle contribution can be distinguished from new physics by comparing atoms or molecules with paired and unpaired electrons.

\subsection{Double Beta Decay}

Neutrinoless double beta decay requires the violation of lepton number and is thus a clear signal for physics beyond the SM. Such a decay could arise from a nonzero Majorana mass for the electron neutrino. In theories with spontaneously broken lepton number, the decay could also be accompanied by the emission of a Majoron. The current half life limit for ${ }^{76} \mathrm{Ge}$ gives a bound on the effective Majorana neutrino mass of $m_{\nu}<1-2 \mathrm{eV}$. The neutrino-Majoron coupling is also constrained to be $g_{\nu, \chi}<10^{-4}$. The current generation of enriched Ge experiments could reach the $0.3 \mathrm{eV}$ level in $m_{\nu}$. A large experiment, NEMO III, using ${ }^{100} \mathrm{Mo}$, is being designed to reach the $0.1 \mathrm{eV}$ level.

\section{Experimental Issues at Hadron Colliders}

The frontiers of particle physics have been significantly pushed forward by discoveries made at colliders with the 
highest available center-of-mass energies. Since the early 1980 's, hadron colliders have become the tool of choice for extending our knowledge of the high energy frontier. The ability of collider detectors to cover a broad range of new phenomena over a wide range of mass scale allows many of the most important measurements to be made and limits to be set in an economical way. A mix of both "discovery" and precision measurements have been successfully carried out at such machines over the last ten years. Progress has depended upon a mix of both theoretical and experimental developments.

During the last ten years, the theoretical community has been able to refine our knowledge of hadron-hadron interactions to enable calculations with increasing precision. The soundness of the theoretical framework puts the hadron-hadron collider on a firm footing for reliable estimation of event rates and backgrounds for a variety of new phenomena.

The experimental community has likewise made important advances during this period. Operating experience at hadron colliders with precision tracking detectors has been gained. These devices open up the possibility of tagging jets containing $b$-quarks, a capability that considerably broadens the physics reach of collider detectors as the recent CDF top quark evidence has shown. In addition, the broad-based program of detector R\&D for SSC and LHC detectors has deepened our understanding of how to handle the high occupancies and radiation fluxes at high luminosity hadron colliders. The knowledge gained from the detector $R \& D$ and design studies gives us confidence that the theoretically predicted physics capabilities of the hadron collider machines can be exploited in detectors that are practical to build and operate.

\subsection{Machine Environment}

The LHC program is designed to study physics beyond the Standard Model over a wide mass range for all types of hypothetical particles. To achieve a substantial reach for the production and observation of new particles, $\mathrm{LHC}$ plans to run at a luminosity of $10^{34} \mathrm{~cm}^{-2} \mathrm{~s}^{-1}$ with a $25 \mathrm{~ns}$ bunch spacing. There will be on average 25 interactions per crossing contributing considerable complexity to the detection of interesting phenomena. Future luminosity upgrades at Fermilab may also generate conditions comparable to these. The challenge met by the SSC and LHC $\mathrm{R} \& \mathrm{D}$ programs was to create detector designs that could survive in these harsh conditions of high occupancy and high radiation, while providing adequate resolution and robust triggering capability.

The general results of the $R \& D$ program showed that existing detector technologies could be successfully employed to meet the SSC/LHC requirements. The higher occupancies expected are accommodated in first order by more finely segmenting the detector. The main $R \& D$ challenges then lay in the area of electronics and DAQ design. The widespread use of custom integrated circuits in the detector front-end electronics allows practical designs to handle the high rates and fine segmentation at finite cost. Modest extension of existing multi-level trigger designs are able to handle the expected event rates.

The high radiation levels in high-luminosity hadron machines have been a cause for concern for both the SSC and LHC experimental groups. Again, the R\&D program has shown that radiation resistant versions of the most sensitive detector elements can be fabricated with only modest extension of existing capability. In particular, many front end electronic circuits that would be exposed to the harsh radiation conditions have been fabricated in radiation-hard versions and shown to work. Also, the level of thermal neutron flux inside the inner tracking cavity and within the detector hall is brought within acceptable limits for detector operation by appropriate shielding design, especially in the region around the machine final focusing quads. Thus, while the problem of radiation resistance remains an ongoing area of further development for the experimenters, solutions to all the most critical problems appear to be in hand. For more specific details on proposed solutions, see the SSC and LHC detector design reports.

\subsection{Detectors}

Two large general purpose detectors are being designed for the LHC, namely ATLAS and CMS. Both experiments have been designed to detect all hypothesized particles accessible to the LHC. Many studies have been carried out by these collaborations, and are detailed in the ATLAS and CMS letters of intent. Generally, to fully exploit the potential physics reach of future hadron colliders, detectors must feature good charged particle tracking, precision electromagnetic calorimetry, full angular coverage for the hadronic calorimetry, and a robust muon detection system.

How physics processes set the requirements on the detector design has been fairly thoroughly explored by the SSC and LHC experimental groups. As examples, detection of the signal $H \rightarrow \gamma \gamma$ for low mass Higgs requires shower counter resolution in the range $\Delta E / E=$ $10 \% / \sqrt{E} \oplus .7 \%$, measurement of photon position with accuracy in the range $\sim 5 \mathrm{~mm} / \sqrt{E}$, and timing resolution adequate for tagging from which bunch the photon originated. Measurement of high mass like-sign $W$ production requires measurement of the sign of the charge of the $W$ decay lepton out to $p_{\perp} \sim 500 \mathrm{GeV} / c$. Angular coverage for both electrons and muons must extend is rapidities of $\sim 2.5$ for good angular acceptance for il!? 
$H \rightarrow 4 \ell$ signals. Many other physics processes have been studied to set detailed design parameters for the detectors.

SSC and LHC R\&D programs were required to show that detectors with the requisite capabilities are practical to construct. The SSC and LHC proposals have shown that detectors with resolutions comparable to existing detectors are adequate for the task. In large measure, the higher event rates expected at future machines are handled by finer segmentation of the detector elements, as mentioned earlier.

The existence of multiple interactions per crossing provides a further complication of this problem, mainly by degrading the pattern recognition capabilities of the detector. Each physics process must be studied with a detailed detector simulation to quantify the capability of the design to detect the signal of interest. Such studies have been carried out in detail, with full event reconstruction, for many of the important Standard Model Higgs detection modes. Numerous other physics processes have also been studied at this level of detail. However, the full extent of the capabilities of the designs as they currently stand has not been explored. In several areas, preliminary parton-level estimations of detector capability will be supplemented by more detailed simulations later. Areas of active investigation by the LHC collaborations include b-jet tagging capability at high luminosity, forward jet tagging capability, and alternate modes for MSSM Higgs detection.

\subsection{Outlook}

During the last five years, important progress has been made in understanding the experimental challenges of hadron colliders. This progress includes improved theoretical understanding of the basic Standard Model physics processes which occur at such machines, improved experimental technique through the operating experience gained mainly at Fermilab, and improved instrumentation mainly through the program of SSC and LHC R\&D. Over the next five years, we expect to maintain a program of continual upgrade at FNAL, and continue preparations for LHC. New limits will be set at FNAL during this period on physics beyond the Standard Model. In addition, measurement of the top and $W$ masses with improved precision will provide indirect information on the electroweak symmetry breaking sector. Within the next ten years, we hope to see the turn-on of the LHC with a first real chance for direct production of more massive Higgs particles, and considerable risk of discovery for physics beyond the Standard Model.

\subsection{The Physics Environment}

Experimental studies at $e^{+} e^{-}$colliders over the past two decades have provided key observations and insights to the nature of the fundamental particles and interactions of the Standard Model. Electron-positron collisions yield events with simple and transparent structures. Annihilation events carry the full energy of the beam, and produce final states with few partons. Searches for new phenomena give complete and unambiguous results, and precision studies of strong and electroweak interactions are made with a minimum of bias and background. The physics environment at $\mathrm{TeV}$-scale colliders will continue to be as ideally suited to the exploration of particle physics [124].

The Standard Model final states that will predominate at a $\sqrt{s}=500 \mathrm{GeV} e^{+} e^{-}$collider are $u d s c b$ pair production (9 units of $R$ ), $W^{+} W^{-}(20$ units of $R), Z Z$ $(1.2$ units of $R$ ), and $t \bar{t}$ ( 1 unit of $R$ ), where $R=87 \mathrm{fb} / \mathrm{s}$ if $\mathrm{s}$ is in $\mathrm{TeV}^{2}$. These processes correspond to relatively simple final states, and, with a typical large solid-angle detector, event structures can be sorted to reduce the 50 or so pions, kaons, and photons to a few 4-vectors that accurately describe the underlying partons. With the partons reconstructed with high precision, topological and kinematic constraints can be utilized to take complete advantage of the simplicity of the physics environment at $e^{+} e^{-}$colliders.

Polarization of the electron beam in a linear collider is a unique tool that provides new and important views of particle physics. It can be utilized to isolate longitudinal W boson states, probe the helicity structure of the interactions of the top quark, reveal the underlying symmetry of SUSY, and determine the gauge structure of new interactions that might be found in high energy collisions - to name just several targets of opportunity. Sources of intense bunches of polarized electrons are presently used at the SLC where beam polarizations of $80 \%$ are routinely delivered to the collision point. Development of sources is continuing and it is reasonable to expect polarizations of $90-95 \%$ in the future. Instrumentation has been developed to measure beam polarization with accuracies of a per cent or better.

\subsection{Detectors}

The analysis of events at future $e^{+} e^{-}$colliders requires good tracking of high-momentum leptons and good reconstruction of quark jets. The isolation of heavy quarks with precision vertex detectors will continue to play an important role in the analysis of events. Particles that go unseen in the detector, such as neutrinos and beramstrahlung photons, can be fully reconstructed in min:1!? 
instances by applying energy-momentum and mass constraints.

Most studies of physics at future electron-positron colliders have used Monte Carlo simulations of detectors with capabilities that have already been achieved at LEP and SLC. The parameters of a "Standard Detector" are listed in Table 12. We see that the charged-particle tracking and electromagnetic and hadronic calorimetery does not extend any technology beyond that now in existence.

Table 12: "Standard Detector" Parameters. All momenta and energies are in $\mathrm{GeV}$. The symbol $\oplus$ means quadratic sum.

\begin{tabular}{|ll|}
\hline Calorimetry & \\
$\sigma_{E} / E$ (electromagnetic) & $8 \% / \sqrt{E} \oplus 2 \%$ \\
$\sigma_{E} / E$ (hadronic) & $60 \% / \sqrt{E} \oplus 2 \%$ \\
Cell size (electromagnetic) & $2^{\circ}$ \\
Cell size (hadronic) & $4^{\circ}$ \\
\hline Tracking & \\
$\sigma_{P_{t}} / P_{t}$ & $10^{-3} \cdot P_{t}$ \\
Vertex resolution (impact) & $20 \mu \mathrm{m} \oplus\left(\frac{100 \mu \mathrm{m}}{P}\right)$ \\
\hline Hermiticity & \\
Calorimetery and tracking & $\theta_{e^{ \pm}}>10^{\circ}$ \\
\hline
\end{tabular}

Table 13: "Improved Detector" Parameters. All momenta and energies are in $\mathrm{GeV}$. The symbol $\oplus$ means quadratic sum.

\begin{tabular}{|ll|}
\hline Calorimetry & \\
$\sigma_{E} / E$ (electromagnetic) & $8 \% / \sqrt{E} \oplus 1 \%$ \\
$\sigma_{E} / E$ (hadronic) & $35 \% / \sqrt{E} \oplus 2 \%$ \\
Cell size (electromagnetic) & $1^{\circ}$ \\
Cell size (hadronic) & $2^{\circ}$ \\
\hline Tracking & \\
$\sigma_{P_{t}} / P_{t}$ & $2 \times 10^{-4} \cdot P_{t}$ \\
Vertex resolution (impact) & $5 \mu \mathrm{m} \oplus\left(\frac{50 \mu \mathrm{m}}{P}\right)$ \\
\hline Hermiticity & \\
Calorimetery and tracking & $\theta_{e^{ \pm}}>10^{\circ}$ \\
\hline
\end{tabular}

We can anticipate that improvements in the techniques and technologies used in the design and construction of particle detectors will occur. At center of mass energies of several hundred $\mathrm{GeV}$, most applications of electromagnetic and hadronic calorimetery do not suffer from lack of shower statistics, but it is increasingly important to minimize systematic errors in the reconstruction of the energies and positions of neutral hadrons and photons. The charged particles of greatest interest are similarly energetic, so that the scattering of tracks in the material of the detector becomes less problematic. The more aggressive set of goals shown in Table 13 might be realizable and certainly would enhance the physics output of the experiment. More systematic and quantitative study is needed to arrive at a complete set of design parameters, but it is clear that existing detectors are not far from ideal.

\subsection{Event Rates and Time Structures}

The total $e^{+} e^{-}$annihilation cross section at high energies is approximately 30 units of $R$, or $10^{-35} \mathrm{~cm}^{2}$ at 500 $\mathrm{GeV}$. Colliders at these energies are designed to generate luminosities typically $5 \times 10^{33} \mathrm{~cm}^{-2} \mathrm{~s}^{-1}$, with an interesting event produced every 10 seconds or so. Small-angle Bhabha scattering of electrons and positrons into the region covered by detector elements (above 10 degrees polar angle) occurs with a cross section 5-10 times the annihilation rate. This serves as a good monitor of the luminosity and energy spectra of collected data samples.

Cross sections for backgrounds produced by the coherent beam-beam interaction and low-energy peripheral two-photon reactions are also larger than those of annihilation events. For example, numerically the total cross section for the reaction $e e \rightarrow$ eeee is $\approx 10^{-26} \mathrm{~cm}^{-2}$ (at beam energy of $500 \mathrm{GeV}$ ). Most of this cross section corresponds to production of $e^{+} e^{-}$pairs near threshold, but the total rate can be sufficiently large that care must be taken in the design of the accelerator and detector to minimize the probablility that soft particles from these interactions will overlap with the desired annihilation events. Accelerator and detector designs have evolved that account for these processes.

The luminosity per bunch crossing and the time interval between crossings varies considerably from one machine design to another. Those based on superconducting linacs yield the lowest luminosity per crossing and spacing between bunches of up to a microsecond. The duty cycle and time structure of these machines are similar to those found at LEP for example, and place similar demands on detector data acquisition systems. Colliders that utilize linacs with high frequency of (at X-Band for example), create brighter collisions in short trains of typically 100 bunches pulsed at repetition rates of 100 $200 \mathrm{~Hz}$. Bunches within a train will be spaced by a few nanoseconds. Detector trigger and data acquisition systems will have long periods to analyze and format information from each repetition cycle, but it will be advantageous for detectors to be able to assign observed charged and neutral particles to their proper bunch-bunch interaction. Detectors have been built with this capability, and these issues have been considered in the evaluation of physics analyses at colliders with center of mass energies as high as $1.5 \mathrm{TeV}$. 


\subsection{Beamstrahlung}

Another property of high-energy linear colliders is that of "beamstrahlung" - the radiation emitted by beam particles as they pass through the electromagnetic field of the opposing bunch. Corrections for the effects of initial-state radiation in $e^{+} e^{-}$collisions has long been a well-understood process, and similar techniques will be needed at future colliders to account for the smearing of annihilation center of mass energies by beamstrahlung. All proposed $e^{+} e^{-}$machines are designed so that the smearing of the center of mass energy by beamstahlung is never greater than, and often less than, the smearing by initial state bremsstrahlung.

The consequences of these effects on the analysis of data have been studied by many groups, and techniques to correct data, and even individual events, for radiation of energy by the incident particles prior to collision have been developed. It is extremely rare that more than one hard photon is emitted in any event, and those that are radiated travel along the incident beam direction, so it is often possible to simply include the radiation as an unknown parameter in a likelihood fit to the event topology. This works well for analyses of exclusive final states such as $W^{+} W^{-}, Z^{0} H^{0}$, and $t \bar{t}$ pairs.

\subsection{Machine-Induced Backgrounds}

While the physics environment created by electronpositron annihilation is remarkably neat and orderly, the single-pass nature of a linear collider poses a very special challenge to the experimenter and machine designer. Unlike a storage ring in which the circulating beam is quickly reduced to only those particles that are wellcontained within the phase space of the physical and dynamic apertures of the machine, a pulsed collider will transmit particles that are very near to its aperture limit. Such particles will create backgrounds in experimental detectors either through the emission of synchrotron radiation as they pass through magnetic fields near the detector, or by the creation of secondary debris when they strike physical elements along the beamline. The detector must be properly masked, and the beam must be properly collimated to remove beam extremeties, or "tails", well upstream of the interaction region. It is important that the design of the collider include accomodation for special optical sections to allow for complete collimation of the phase space of the beam.

The experience of the MARK II and SLD detectors [125] at the SLC has been an invaluable guide to the problems that will be encountered by experiments at future linear colliders. Successful operation of these experiments and the lessons that have been learned from the SLC provide a good basis for design and operation of experiments at the NLC.

\section{Conclusions}

Exploration of the $\mathrm{TeV}$-scale requires a new generation of colliders and detectors beyond those currently in operation, and is essential for the long-term vitality of particle physics. In this report, we describe an in-depth study of the phenomenology of electroweak symmetry breaking and quantify the "physics reach" of present and future colliders. Our investigations focus on the Standard Model (with one Higgs doublet) and beyond: models of low-energy supersymmetry, dynamical electroweak symmetry breaking, and other approaches containing new particles and interactions. Implications for future experiments at hadron and $e^{+} e^{-}$colliders are considered. On the basis of this work, we present the following conclusions and recommendations.

The participation of the United States in the LHC program should be vigorously supported. An extensive study of the electroweak symmetry breaking sector and the elucidation of physics beyond the Standard Model will require a robust experimental program at the LHC. The LHC possesses an impressive discovery potential for Higgs bosons, low-energy supersymmetry, and a large variety of beyond the Standard Model phenomena, with a mass reach in many cases five to ten times the corresponding mass reach at existing facilities. Of course, with the LHC approximately ten years from its initial run, one should not overlook the possibility that initial evidence for physics associated with electroweak symmetry breaking and/or physics beyond the Standard Model could be uncovered at upgrades of existing facilities.

Detailed simulations of high energy particle physics processes at hadron colliders are often required to obtain an accurate assessment of the new physics discovery capabilities of future experiments. These include realistic treatments of hadronic jets (beyond parton-level Monte Carlo analyses) and full detector simulations. An active collaboration between experimentalists and theorists is crucial to the further development of strategies to extract evidence for electroweak symmetry breaking and beyond the Standard Model physics at future colliders.

There are many instances of the complementarity of future hadron colliders and future $e^{+} e^{-}$colliders. A high energy $e^{+} e^{-}$collider will provide a unique lowbackground environment for the study of the production and decay of heavy gauge bosons and top quarks and of new physics beyond the Standard Model. A Next Linear Collider (NLC) at $0.5 \mathrm{TeV}$ is an ideal laboratory for the study of intermediate-mass Higgs bosons, while at $1.5 \mathrm{TeV}$, it can begin to probe the physics of a stronglycoupled electroweak symmetry breaking sector. Experimental studies at the NLC would provide outstanding opportunities for discovery, while significantly enhancing the ability of the LHC to interpret evidence for new physics. 
Other possible future collider scenarios are beginning to be examined. Physics studies at a number of hypothetical Tevatron upgrades beyond the Main Injector have been initiated. A full study of the scientific potential of such machines is necessary to provide input into any future cost/benefit evaluation that might be undertaken in the future.

In plotting out the course for future colliders, the US must work closely with its international partners. By avoiding the duplication of resources and taking into account the complementarity of various approaches, it should be possible to propose a set of future facilities that can fully explore the $\mathrm{TeV}$ energy scale and elucidate the physics of electroweak symmetry breaking. In doing so, we can push the frontiers of particle physics forward and keep our field intellectually vital and exciting.

\section{References}

[1] Electroweak Symmetry Breaking and Physics Beyond the Standard Model, eds. T. Barklow, S. Dawson, H.E. Haber and J. Siegrist, (World Scientific, Singapore) to be published.

[2] For convenient summaries of early material on the SM and MSSM Higgs sectors, see J.F. Gunion, H.E. Haber, G.L. Kane and S. Dawson, The Higgs Hunters Guide (Addison Wesley, Redwood City, CA, 1990). For more recent theoretical and phenomenological developments see Perspectives on Higgs Physics, ed. G. Kane (World Scientific, River Edge, N.J., 1992).

[3] H.E. Haber and R. Hempfling, Phys. Rev. Lett. 66, 1815 (1991); Phys. Rev. D48, 4280 (1993); Y. Okada, M. Yamaguchi and T. Yanagida, Prog. Theor. Phys. 85, 1 (1991); Phys. Lett. B262, 54 (1991); J. Ellis, G. Ridolfi and F. Zwirner, Phys. Lett. B257, 83 (1991); B262, 477 (1991); R. Barbieri, M. Frigeni, and F. Caravaglios, Phys. Lett. B258, 167 (1991).

[4] For a review, see e.g., P. Janot, in Electroweak Interactions and Unified Theories, 27th Rencontres de Moriond, Les Arcs, France, March 1992, ed. J. Tran Thanh Van (Gif-sur-Yvette, France, Editions Frontieres, 1992) Vol. 1, p. 317.

[5] J. Gunion, in Properties of SUSY Particles, Proceedings of the 23rd Workshop of the INFN Eloisatron Project, Erice, Italy, Sept. 28-Oct. 4, 1992, eds. L. Cifarelli and V. Khoze, (World Scientific, River Edge, N.J., 1993) p. 279.

[6] For a review and references, see J. Gunion, Nucl. Phys. B (Proc. Suppl.) 37B, 253 (1994), Proceedings of the Zeuthen Workshop on Elemen- tary Particle Theory - LEP 200 and Beyond, Teupitz/Brandenburg, Germany, 10-15 April 1994, eds. T. Riemann and J. Blumlein.

[7] S. Mrenna and G. Kane, CALT-68-1938 (1994).

[8] ATLAS Technical Proposal, CERN/LHCC/94-43 LHCC/P2 (1994).

[9] CMS Technical Proposal, CERN/LHCC/94-38, LHCC/P1 (1994).

[10] H. Baer and X. Tata, in Properties of SUSY Particles, Proceedings of the 23rd Workshop of the INFN Eloisatron Project, Erice, Italy, Sept. 28Oct. 4, 1992, eds. L. Cifarelli and V. Khoze, (World Scientific, River Edge, N.J., 1993) p. 244.

[11] F. Zwirner, ibid., p. 509.

[12] For recent studies, see P. Grosse-Wiesemann, D. Haidt, and H.J. Schreiber, in Workshop on $e^{+} e^{-}$ Collisions at $500 \mathrm{GeV}$ : the Physics Potential, Munich, February; Annecy, June; Hamburg, September 1991, ed. P.M. Zerwas, DESY 92-123A (1992), p. 37; P. Janot, ibid., p. 107; A. Djouadi, D. Haidt, B.A. Kniehl, B. Mele, and P.M. Zerwas, ibid., p. 11.

[13] J.F. Gunion, in Proceedings of the 2nd International Workshop on "Physics and Experiments with Linear $e^{+} e^{-}$Colliders", Waikoloa, HI, 1993, eds. F. Harris, S. Olsen, S. Pakvasa and X. Tata, Waikoloa, (World Scientific, Singapore, 1994) p. 166.

[14] P. Janot, ibid., p. 192.

[15] A. Djouadi, J. Kalinowski, P.M. Zerwas, Z. Phys. C57, 569 (1993); A. Brignole, J. Ellis, J.F. Gunion, M. Guzzo, F. Olness, G. Ridolfi, L. Roszkowski and F. Zwirner, in Workshop on $e^{+} e^{-}$Collisions at 500 GeV: The Physics Potential, Munich, February; Annecy, June; Hamburg, September 1991, ed. P.M. Zerwas, DESY 92-123A/B, p. 613.

[16] V. Barger, K. Cheung, A. Djouadi, B.A. Kniehl, and P.M. Zerwas, Phys. Rev. D49, 79 (1994).

[17] B. Grzadkowski and J.F. Gunion, Phys. Lett. B294, 361 (1992), and preprint UCD-95-5 (hep$\mathrm{ph} / 9501339]$.

[18] M. D. Hildreth, T. L. Barklow, and D. L. Burke, Phys. Rev. D49, 3441 (1994).

[19] P. Langacker and M. Luo, Phys. Rev. D44, 817 (1991); J. Ellis, S. Kelley and D.V. Nanopoulos, Phys. Lett. B260, 131 (1991); U. Amaldi, W. de Boer and H. Fürstenau, Phys. Lett. 260B, 447 (1991). 
[20] L.E. Ibáñez and G.G. Ross, Phys. Lett. 110B, 215 (1982); K. Inoue et. al., Prog. Theor. Phys. 68, 927 (1982); J. Ellis, D.V. Nanopoulos and K. Tamvakis, Phys. Lett. 121B, 123 (1983); L. Alvarez-Gaumé, J. Polchinski and M.B. Wise, Nucl. Phys. B221, 495 (1983).

[21] L. Girardello and M.T. Grisaru, Nucl. Phys. B194, 65 (1982).

[22] For reviews, see H.P. Nilles, Phys. Rep. 110, 1 (1984); P. Nath, R. Arnowitt and A. Chamseddine, Applied $N=1$ Supergravity, ITCP Series in Theoretical Physics (World Scientific, Singapore, 1984), Vol. 1.

[23] See e.g., G.L. Kane, C. Kolda and J.D. Wells, Phys. Rev. Lett 70, 2686 (1993) and references therein.

[24] M. Drees, Phys. Lett. B181, 279 (1986); J.S. Hagelin and S. Kelley, Nucl. Phys. B342, 95 (1990).

[25] Y. Nir and N. Seiberg, Phys. Lett. B309, 337 (1993).

[26] T. Elliott, S.F. King and P.L. White, Phys. Rev. D49, 2435, 1994; U. Ellwanger, M. Rausch de Traubenberg and C.A. Savoy, Phys. Lett. B315, 331 (1993).

[27] L.E. Ibáñez and G.G. Ross, Nucl. Phys. B368, 3 (1992).

[28] H. Haber and G. Kane, Phys. Rep. 117, 75 (1985); $\mathrm{X}$. Tata, in The Standard Model and Beyond, p. 304, edited by J. Kim (World Scientific, Singapore, 1991); R. Arnowitt and P. Nath, Lectures presented at VII J. A. Sweica Summer School, Campos do Jordao, Brazil, 1993, CTP-TAMU$52 / 93$.

[29] F. Paige and S. Protopopescu, in Supercollider Physics, p. 41, ed. D. Soper (World Scientific, Singapore, 1986); H. Baer, F. Paige, S. Protopopescu and X. Tata, in Proceedings of the Workshop on Physics at Current Accelerators and Supercolliders, ed. J. Hewett, A. White and D. Zeppenfeld, (Argonne National Laboratory, 1993).

[30] H. Baer, A. Bartl, D. Karatas, W. Majerotto and X. Tata, Int. J. Mod. Phys. A4, 4111 (1989).

[31] H. Baer, C. H. Chen, C. Kao and X. Tata, FSUHEP-950301 (1995); H. Baer, C.H. Chen, F. Paige and X. Tata, Phys. Rev. D50, 4508 (1994).

[32] T. Kamon, J. Lopez, P. McIntyre and J. White, Phys. Rev. D50, 5676 (1994).
[33] H. Baer, C. Kao and X. Tata, Phys. Rev. D48, 2978 (1993).

[34] H. Baer, J. Sender and X. Tata, Phys. Rev. D50, 4517 (1994).

[35] V. Barger, M. Berger, P. Ohmann and R. Phillips, Phys. Rev. D50, 4299 (1994); H. Baer. C. Kao and X. Tata, Phys. Rev. D51, 2180 (1995).

[36] H. Baer, C. H. Chen, F. Paige and X. Tata, Phys. Rev. D 49, 3283 (1994).

[37] H. Baer, C. H. Chen, R. Munroe. F. Paige and X. Tata, Phys. Rev. D51, 1046 (1995).

[38] H. Baer, J. Gunion, C. Kao and H. Pois, Phys. Rev. D51, 2159 (1995).

[39] For example, see, J.-F. Grivaz, in Properties of SUSY Particles, Proceedings of the 23rd Workshop of the INFN Eloisatron Project, Erice, Italy, Sept. 28-Oct. 4, 1992, eds. L. Cifarelli and V. Khoze, (World Scientific, River Edge, N.J., 1993).

[40] J.L. Feng and M.J. Strassler, SLAC-PUB-6497 [hep-ph/9408359].

[41] R. Becker and C. Van der Velde, in Proceedings of European Meeting of the Working Groups on Physics and Experiments at Linear $e^{+} e^{-}$Colliders, ed. by P.M. Zerwas, DESY-93-123C.

[42] J.-F. Grivaz, in Proceedings of Physics and Experiments with Linear Colliders, Saariselkä, Finland, September 1991, eds. R. Orava, P. Eerola, and M. Nordberg (World Scientific, Singapore, 1992).

[43] T. Tsukamoto, K. Fujii, H. Murayama, M. Yamaguchi, and Y. Okada, Phys. Rev. D51, 3153 (1995).

[44] JLC Group, “JLC-I”, KEK-Report 92-16 (1992).

[45] A. Bartl, W. Majerotto and A. Sopczak, in preparation.

[46] R. Casalbuoni, P. Chiappetta, S. De Curtis, F. Feruglio, R. Gatto, B. Mele, and J. Terron, Phys. Lett. B249, 130 (1991).

[47] M. S. Chanowitz and W. Kilgore, Phys. Lett. B322, 147 (1994); B347, 387 (1995).

[48] J. Bagger, V. Barger, K. Cheung, J.F. Gunion, T Han, G. Ladinsky, R. Rosenfeld, and C.-P. Yuan, Phys. Rev. D49, 1246 (1994); new analyses in progress. 
[49] M.E. Peskin, in Proceedings of Physics and Experiments with Linear Colliders, Saariselkä, Finland, September 1991, eds. R. Orava, P. Eerola, and M. Nordberg (World Scientific, Singapore, 1992).

[50] T. Barklow, talk presented at Albuquerque DPF 94, SLAC-PUB-6618.

[51] R. Casalbuoni et al., in Proceedings of the 2nd International Workshop on "Physics and Experiments with Linear $e^{+} e^{-}$Colliders", Waikoloa, HI, 1993, eds. F. Harris, S. Olsen, S. Pakvasa and X. Tata, Waikoloa, (World Scientific, Singapore, 1994) p. 887.

[52] V. Barger, K. Cheung, T. Han, and R. Phillips, MAD-PH-95-865 [hep-ph/9501379].

[53] R. S. Chivukula and M. Golden, Phys. Lett. B267, 233 (1991).

[54] M. Berger, contribution to this report.

[55] S. J. Brodsky, talk presented at the 2nd International Workshop on Physics and Experiments with Linear $e^{+} e^{-}$Colliders, Waikoloa, HI, April 1993.

[56] G. Jikia, IFVE-94-77 [hep-ph/9407393].

[57] K. Cheung, Phys. Rev. D50, 4290 (1994).

[58] L. Susskind, Phys. Rev. D20, 2619 (1979); S. Weinberg, Phys. Rev. D13, 974 (1976); D19, 1277 (1979).

[59] E. Eichten, I. Hinchliffe, K. Lane and C. Quigg, Rev. Mod. Phys. 56, 579 (1984) [E: 58, 1065 (1986)]; E. Eichten, I. Hinchliffe, K. Lane and C. Quigg, Phys. Rev. D34, 1547 (1986).

[60] GEM Technical Design Report, SSCL-SR-1219, 1993.

[61] A. Manohar and L. Randall, Phys. Lett. B246, 537 (1990); L. Randall and E. H. Simmons, Nucl. Phys. B380, 3 (1992); V. Lubicz, Nucl. Phys. B404, 559 (1993).

[62] K. Lane and M. V. Ramana, Phys. Rev. D44, 2678 (1991).

[63] V. Lubicz, work in progress.

[64] J.F. Gunion and H.E. Haber, Phys. Rev. D48, 5109 (1993); D.L. Borden, D.A. Bauer and D.O. Caldwell, Phys. Rev. D48, 4018 (1993).

[65] V. Barger, R.J.N. Phillips and D.P. Roy, Phys. Lett. B324, 236 (1994); J. F. Gunion, Phys. Lett. B322, 125 (1994).
[66] E. Eichten and K. Lane, Phys. Lett. B327, 129 (1994); K. Lane, BUHEP-94-12 (1994) [hep$\mathrm{ph} / 9406344]$.

[67] R.S. Chivukula, M. Golden and E.H. Simmons, Nucl. Phys. B363, 83 (1991).

[68] J.L. Hewett and T.G. Rizzo, Phys. Rep. 183, 193 (1989) and references therein.

[69] For a review and original references see R.N. Mohapatra, Unification and Supersymmetry (Springer, New York, 1986).

[70] P. Langacker and M. Luo, Phys. Rev. D44, 817 (1991).

[71] F. Abe et al. (CDF Collaboration), Phys. Rev. Lett. 74, 2900 (1995).

[72] S. Godfrey, Phys. Rev. D51, 1402 (1995).

[73] F. del Aguila, M. Cvetič, and P. Langacker, in Proceedings of the 2nd International Workshop on "Physics and Experiments with Linear $e^{+} e^{-}$Colliders", Waikoloa, HI, 1993, eds. F. Harris, S. Olsen, S. Pakvasa and X. Tata, Waikoloa, (World Scientific, Singapore, 1994) p. 490 and p. 929.

[74] For reviews on exotic fermions see, A. Djouadi, D. Schaile and C. Verzegnassi [conv.] et al, Proceedings of the Workshop on $e^{+} e^{-}$Collisions at $500 \mathrm{GeV}$ : the Physics Potential, Report DESY 92$123 \mathrm{~A} / \mathrm{B}, \mathrm{P}$. Zerwas, ed; P. Langacker and D. London, Phys. Rev. D38, 244 (1988).

[75] J. Maalampi and M. Roos, Phys. Rep. 186, 53 (1990).

[76] R. Robinett and J. Rosner, Phys. Rev. D25, 3036 (1982); P. Langacker, R. W. Robinett and J. L. Rosner, Phys. Rev. D30, 1470 (1984); V. Barger et al., Phys. Lett. B118, 68 (1982); W. Buchmüller and C. Greub, Nucl. Phys. B363, 345 (1991 and)Nucl. Phys. B381, 109 (1992).

[77] For a recent discussion on exotic fermions properties, decays and production at $e^{+} e^{-}$colliders, and for references on previous work, see A. Djouadi, Z. Phys. C63, 317 (1994); G. Azuelos and A. Djouadi, Z. Phys. C63, 327 (1994).

[78] W. Buchmüller, R. Rückl, and D. Wyler, Phys. Lett. B191, 442 (1987); W. Buchmüller, and D. Wyler, Phys. Lett. B177, 377 (1986).

[79] P.H. Frampton and D. Ng, Phys. Rev. D45, 4240 (1992); P.H. Frampton et al., Phys. Lett. B317, 369 (1993); J. Agrawal, P.H. Frampton, and D. Ng, Nucl. Phys. B386, 267 (1992); T.G. Rizzo, Phys. Rev. D45, 42 (1992); D46, 910 (1992). 
[80] For reviews on fermion substructure, see: $\mathrm{M}$. Peskin, Proceedings of the 10th International Symposium on Lepton-Photon Interactions at High Energies, Bonn FRG, September, 1981, ed. W. Pfeil (Bonn University, Physik Inst., 1981); H. Terazawa, Proceedings of the Europhysics Conference on Flavor Mixing in Weak Interactions, Erice Italy, March 1984, ed. Ling-Lie Chau (Plenum Press, New York, 1984); W. Buchmüller, Lectures at the 24th Int. Wochenuniversität für Kernphysik, Schladming, Austria, 1985, eds. H. Mitter and W. Plessas (Springer-Verlag, Vienna, 1985); R. Peccei, Proceedings of the Lake Louise Winter Institute, "Selected Topics in Electroweak Interactions" Lake Louise, Canada, February 1987, eds. J.M. Cameron et al. (World Scientific, Singapore, 1987); F. Boudjema, Int. J. Mod. Phys. A6 (1991) 1.

[81] For a detailed analysis of excited fermions properties and production at $e^{+} e^{-}$and $e p$ colliders, and a complete set of references on previous work, see $F$. Boudjema, A. Djouadi and J. L. Kneur, Z. Phys. C57, 425 (1992); K. Hagiwara, S. Komamiya and D. Zeppenfeld, Z. Phys. C29,115 (1985).

[82] Excited fermions at $p p$ colliders are discussed in: U. Baur, M. Spira, P.M. Zerwas, Phys. Rev. D42, 815 (1990); U. Baur, I. Hinchliffe and D. Zeppenfeld, Proceedings of the Workshop From Colliders to Supercolliders, Madison, May 1987, eds. V. Barger and F. Halzen (World Scientific, Singapore, 1987).

[83] A. Djouadi, contribution to this report.

[84] M. Drees, Nucl. Phys. B298, 333 (1988); W. Buchmüller, C. Greub and P. Minkowski, Phys. Lett. B267, 395 (1991).

[85] M. Chanowitz, M. Furman and I. Hinchliffe, Phys. Lett. B78, 285 (1978); F. Csikor and I. Montvay, Phys. Lett. B231, 503 (1990).

[86] E. Nardi, E. Roulet and D. Tommasini, contribution to this report.

[87] M. Davier, Rapporteur talk at the Lepton-Photon Conference, Geneva 1991.

[88] G. Bhattacharya, P. Kalyniak and K. Peterson, contribution to this report.

[89] M. Spira, contribution to this report.

[90] C. Greub, contribution to this report.

[91] T. G. Rizzo, contribution to this report.

[92] J. Blümlein, contribution to this report.
[93] G. Bélanger, M. Doncheski, S. Godfrey, D. London, H. Nadeau, contribution to this report.

[94] J. Hewett, T. Rizzo, S. Pakvasa, A. Pomarol, and H.E. Haber, contribution to this report.

[95] P. Chiappetta, contribution to this report.

[96] N. Leporé, B. Thorndyke, H. Nadeau and D. London, contribution to this report.

[97] J. L. Kneur, contribution to this report.

[98] E. Eichten, K. Lane and M.E. Peskin, Phys. Rev. Lett. 50, 811 (1983).

[99] K. Hagiwara et al., Nucl. Phys. B282, 253 (1987).

[100] A. de Rujula et al., Nucl. Phys. B384, 3 (1992) C. Arzt, M.B. Einhorn, and J. Wudka, Phys. Rev. D49, 1370 (1994); Nucl. Phys. B433, 41 (1994); C.P. Burgess, M. Frank and C. Hamzaoui, MCGILL-93-40 [hep-ph/9312238] and references therein.

[101] K. Hagiwara et al., Phys. Rev. D48, 2182 (1993).

[102] F. Abe et al. (CDF Collaboration), Phys. Rev. Lett. 74, 1936 (1995); 1941 (1995); S. Errede, FERMILAB-Pub-94/306-E preprint (September 1994), to appear in the Proceedings of the 27th International Conference on High Energy Physics, Glasgow, Scotland, July 20-27, 1994; J. Ellison (D $\emptyset$ Collaboration), FERMILAB-Conf-94/329-E preprint (November 1994) to appear in the Proceedings of the DPF'94 Conference, Albuquerque, NM, August 1994; H. Aihara, talk given at the International Symposium on Vector Boson SelfInteractions, Los Angeles, CA, February 1995.

[103] M. Acciarri et al. (L3 Collaboration), Phys. Lett. B346, 190 (1995).

[104] A. Miyamoto, in Proceedings of the 2nd International Workshop on "Physics and Experiments with Linear $e^{+} e^{-}$Colliders", Waikoloa, HI, 1993 , eds. F. Harris, S. Olsen, S. Pakvasa and X. Tata, Waikoloa, (World Scientific, Singapore, 1994) p. 141.

[105] F. Abe et al. (CDF Collaboration), Phys. Rev. D50, 2966 (1994).

[106] F. Abe et al. (CDF Collaboration), Phys. Rev. Lett. 73; 225 (1994).

[107] F. Abe et al. (CDF Collaboration), FERMILABPUB-95/022-E (1995).

[108] S. Abachi et al. (D0 Collaboration), FERMILABPUB-95/028-E (1995). 
[109] C. T. Hill and S. J. Parke, Phys. Rev. D49, 4454 (1994).

[110] G. Kane, G. A. Ladinsky, and C.-P. Yuan, Phys. Rev. D45, 124 (1992); D. Atwood and A. Soni, Phys. Rev. D45, 2405 (1992); G. A. Ladinsky and C.-P. Yuan, Phys. Rev. D49, 4415 (1994); D. O. Carlson, E. Malkawi, and C.-P. Yuan, Phys. Lett. B337, 145 (1994); E. Malkawi and C.-P. Yuan, Phys. Rev. D50, 4462 (1994); T. Barklow and C. Schmidt, contribution to the 1994 DPF conference.

[111] R. S. Chivukula, S. B. Selipsky, and E. H. Simmons, Phys. Rev. Lett. 69, 575 (1992); R. S. Chivukula, E. H. Simmons, and J. Terning, Phys. Lett. B331, 383 (1994).

[112] T.G. Rizzo, Phys. Rev. D50, 4478 (1994).

[113] D. Atwood, A. Kagan, and T. G. Rizzo, SLACPUB-6580 (1994).

[114] C-P. Yuan et al, in Proceedings of the Workshop on Physics at Current Accelerators and Supercolliders, eds. J. Hewett, A. White, and D. Zeppenfeld (Argonne, 1993).

[115] D. Amidei, in preparation.

[116] T.G. Rizzo, Phys. Rev. D51, 1064 (1995).

[117] K. Fujii, T. Matsui, and Y. Sumino, Phys. Rev. D50, 4341 (1994).
[118] C. Schmidt and M. E. Peskin, Phys. Rev. Lett. 69, 410 (1992).

[119] G. Bagliese, et al., in Proceedings of the Workshop on $e^{+} e^{-}$Collisions at $500 \mathrm{GeV}$, ed. P. M. Zerwas (DESY, 1992).

[120] M. Hildreth, in preparation.

[121] M. E. Peskin and T. Takeuchi, Phys. Rev. Lett. 65, 964 (1990); Phys. Rev. D46, 381 (1992).

[122] I. Maksymyk, C. P. Burgess, and D. London, Phys. Rev. D50, 529 (1994).

[123] For a review of implications of non-SM physics in $b \rightarrow s \gamma$, see J.L. Hewett, 21st Annual SLAC Summer Institute on Particle Physics, Stanford, CA, July 1993.

[124] For a review, see: D.L. Burke, in Workshop on Physics and Experiments with Linear $e^{+} e^{-}$Colliders, Saariselkä, Finland, September 1991; and W. Ash, Proceedings of the 2nd International Workshop on "Physics and Experiments with Linear $e^{+} e^{-}$Colliders", Waikoloa, HI, 1993, eds. F. Harris, S. Olsen, S. Pakvasa and X. Tata, Waikoloa, (World Scientific, Singapore, 1994).

[125] R. Jacobsen, et al., SLAC-PUB-5205, April, 1990. 